\title{
NOVEL SPECT RNA QUANTIFICATION OF MECHANICAL DYSSYNCHRONY FOR THE PREDICTION OF CRT RESPONSE
}

by

Michel Lalonde, M.Sc.

A thesis submitted to the Faculty of Graduate and Postdoctoral Affairs in partial fulfillment of the requirements

for the degree of

Doctor of Philosophy

Ottawa-Carleton Institute for Physics

Department of Physics, Carleton University

Ottawa, Ontario, Canada

September 2013

Copyright (C) 2013, Michel Lalonde 


\section{Abstract}

Mechanical dyssynchrony (MD) occurs when different regions of the heart wall contract out of phase, reducing the pumping efficiency of the heart and possibly leading to congestive heart failure (CHF). Cardiac resynchronization therapy (CRT) is a possible medical intervention for CHF that resynchronizes the heart, effectively reducing MD. However, CRT has been shown to provide no clinical benefit in $20-50 \%$ of recipients. Current CRT patient selection protocols do not include assessment of MD despite evidence that the mechanism of CRT response is to improve MD. Single-photon emission computed tomography (SPECT) radionuclide angiography (RNA) is an imaging technique that is capable of quantifying MD by accurately assessing cardiac wall motion.

In this thesis, new methods of quantifying MD with SPECT RNA were developed and evaluated as a means to predict response to CRT.

Novel Fourier-analysis (FA) amplitude values were compared to left ventricular (LV) scar size in the lateral wall to determine whether they can serve as surrogate markers for predicting response to CRT. Moderate but significant correlations $(\mathrm{r}=0.51, \mathrm{p} \leq 0.05)$ were shown to exist between amplitude- and scar-based parameters. Lateral wall amplitude 
analysis was equivalent to lateral wall scar analysis for predicting response to CRT, but there were indications that amplitude may also be partly complementary to scar.

Novel MD parameters were developed using FA amplitude parameters and by developing novel clustering algorithms. These novel MD parameters were evaluated alongside preestablished FA phase-based parameters in both global and segmental regions for their ability to predict CRT response. Using FA, septal wall amplitude standard deviation (SD), global synchrony and global phase-SD were significantly predictive of CRT response. Similar to the FA amplitude results, cluster analysis was predictive of CRT response using septal wall analysis. Based on the area under a receiver operating characteristic curve (AUC), there was an indication of improved predictive ability using cluster analysis $(\mathrm{AUC}=0.82)$ over scar analysis $(\mathrm{AUC}=0.73)$ and $\mathrm{FA}(\mathrm{AUC}=0.78)$, but differences were not significant in the small test population.

Novel amplitude-based FA parameters and cluster analysis approaches provide promising tools for the assessment of MD and the prediction of CRT response with SPECT RNA. 


\section{Statement of Originality}

This thesis describes the author's research accomplished at The Ottawa Hospital and the University of Ottawa Heart Institute (UOHI) during the course of the Ph.D. program at Carleton University.

The PREDICT study (1) led by principal investigator Dr. David Birnie and the RAFT study $(2,3)$ led by principal investigators, Dr. Anthony Tang and Dr. George Wells are both UOHI studies. The thesis author had no involvement in study design, patient assessment, CRT selection criteria; baseline scans or follows up assessments. Acquisitions were reconstructed following standard protocol by UOHI technicians. Positron emission tomography (PET) scar assessment was evaluated using FlowQuant (C) software (UOHI) by qualified UOHI technicians. Functional parameters such as left ventricular ejection fraction (LVEF) were analyzed with planar radionuclide angiography (RNA) acquisitions using the FUGA software (Hermes Medical Solutions) by qualified UOHI technicians. The statistical analysis of the double-blind RAFT data was performed by the Cardiovascular Research Methods Centre led by Dr. George Wells at the UOHI. SPECT RNA software was written by the thesis author during his Master's thesis, with occasional improvements during his doctoral studies. 
The thesis author analyzed all SPECT RNA patient data referred to in this thesis, including LV segmentation and subsequent phase and cluster analysis. The thesis author is responsible for the creation, development and implementation of novel FA parameters and cluster analysis methodologies. Statistical analysis was performed by the thesis author on both the normal population and PREDICT data set. All thesis content, including pictures, graphs and tables were produced by the author unless otherwise specified.

Some of the methodology and results described in this thesis have been published as abstracts and presented at conferences, such as the Society of Nuclear Medicine (SNM) annual meeting, and submitted to journals for publication. They are outlined below:

Lalonde M, Ruddy TD, Birnie D, Wassenaar R, and Wells RG. Comparison of SPECT RNA phase analysis amplitude values and left ventricular lateral wall scar sizes, $J . \mathrm{Nucl}$. Med. 53 (suppl.) 1832P (2012) [abstract] - 2012 SNM Annual Meeting.

- The methods and results presented in this abstract constitute a large part of the research presented in chapter 3. However, the amplitude parameters used in this thesis were updated from those in the abstract to better relate with scar definitions.

- The author identified several new amplitude indices and implemented software for semi-automatic extraction of the measures from SPECT RNA studies. The author performed the data analysis comparing the amplitude and scar parameters as well as determined the ability of the amplitude parameters to predict CRT 
response. The thesis author also prepared and presented the abstract and poster for presentation.

Lalonde M, Birnie D, Ruddy TD, deKemp RA, Beanlands RSB, Wassenaar R, and Wells RG. SPECT gated blood pool phase analysis of lateral wall motion for prediction of CRT response. Submitted to Journal of Nuclear Medicine in June 2013.

- The work in this paper is presented in chapter 3.

- The author identified several new amplitude indices and implemented software for semi-automatic extraction of the measures from SPECT RNA studies. The thesis author performed the data analysis comparing the amplitude and scar parameters as well as determined the ability of the amplitude parameters to predict CRT response. The thesis author also prepared the article for publication.

Lalonde M, Ruddy TD, Birnie D, Wells RG, and Wassenaar R. Quantifying variation in normal cardiac wall-motion curves, J. Nucl. Med. 52 (suppl.) 1169P (2011) [abstract] 2011 SNM Annual Meeting.

- This abstract describes the preliminary methodology of cluster analysis which is presented in chapter 5. Normal wall motion curves as well as the best normalization techniques to minimize inter- and intra-patient variability are defined.

- The thesis author developed the approach to RNA cluster analysis that is detailed in Chapter 5 and implemented software for semi-automatic wall-motion cluster analysis of SPECT RNA studies. The thesis author analyzed the patient data to 
create the normal wall motion curves and to minimize the variability between curves. The author created and presented the abstract and poster.

Lalonde M, Birnie D, Ruddy TD, Wassenaar R, and Wells RG. Development and optimization of SPECT RNA cluster analysis for the prediction of CRT outcome, J. Nucl. Med. 54 (suppl.2) 1678P (2013) [abstract] - 2013 SNMMI Annual Meeting.

- The remaining methodology of cluster analysis (chapter 5) was presented in this poster along with the results of cluster analysis's ability to predict response to CRT.

- In the abstract, results from cluster analysis are compared to PET scar size (chapter 3) and FA results (chapter 4).

- The thesis author is responsible for the design, creation and implementation of cluster analysis and its various metrics. The thesis author also performed the data analysis for determining the ability of cluster analysis at predicting response to CRT.

- The thesis author prepared and presented the abstract and poster for presentation.

Lalonde M, Wells R G, Birnie D, Ruddy TD, and Wassenaar R. Development and optimization of SPECT gated blood pool cluster analysis for the prediction of CRT outcome. Submitted to the journal Medical Physics in June 2013.

- This paper comprises the work presented in Chapter 5 and integrates the work from the previous two abstracts. 
- The thesis author designed, created and implemented the cluster analysis technique and software and performed all the analysis as described in the previous two abstracts.

- The thesis author prepared the article for submission. 


\section{Acknowledgements}

I would like to thank both of my supervisors, Dr. Glenn Wells and Dr. Richard Wassenaar for their support and direction during the past several years. In particular, I would like to thank Dr. Wells for accepting to guide me and my project midway through my Ph.D. research. I have learned a lot under both of their guidance. Thanks you as well to Dr. Terrence Ruddy and Dr. David Birnie. This research would not have been possible without their input and guidance.

I would also like to acknowledge the staff at both The Ottawa Hospital and the University of Ottawa Heart Institute. A special thanks to Brian Marvin and the rest of technologists who have helped me during my research. Thank you to Dr. George Wells, Li Chen and the rest of the Cardiovascular Research Methods Center for sharing their expertise in data analysis.

I would like to thank my wife, Jacynthe, for her love, patience and encouragement during these past years. Thank you as well to my family and friends, in particular to my parents for their love and support. Finally, to my little girl-to-be, you have already impacted my life more than I could imagine and have provided me with excitement, renewed faith and motivation these last few months. Merci! Je vous aime. 


\section{Table of Contents}

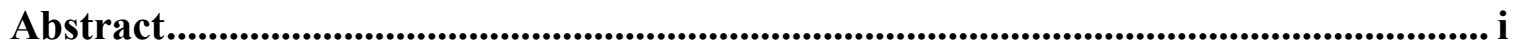

Statement of Originality ................................................................................................................ iii

Acknowledgements ..................................................................................................................... viii

Table of Contents ........................................................................................................................... ix

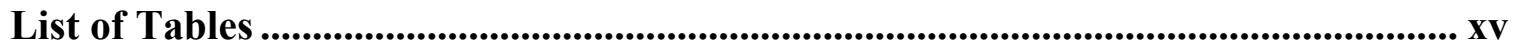

List of Figures........................................................................................................... xvii

List of Acronyms ............................................................................................................. $\mathrm{xx}$

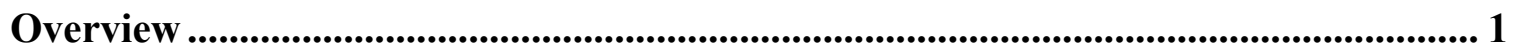

Chapter 1 Medical Background and Motivation .................................................................. 4

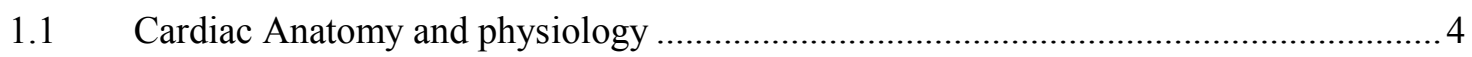

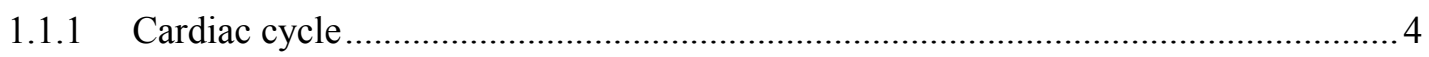

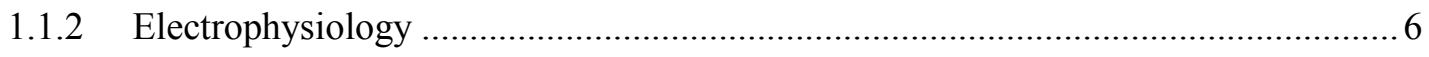

$1.2 \quad$ Ventricular Dyssynchrony ............................................................................ 8

1.2.1 Electrical vs. mechanical dyssynchrony..........................................................

$1.3 \quad$ Congestive Heart Failure (CHF).......................................................................... 11

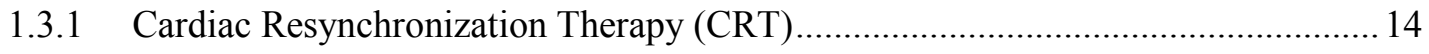

Chapter 2 Imaging techniques for quantification of dyssynchrony ............................. 21

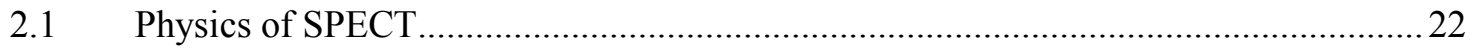

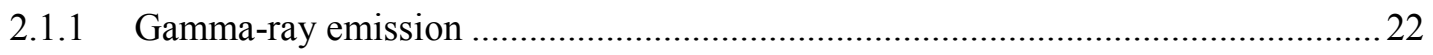




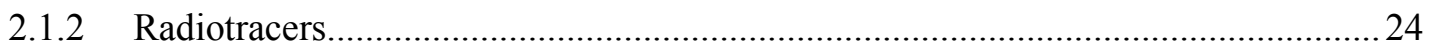

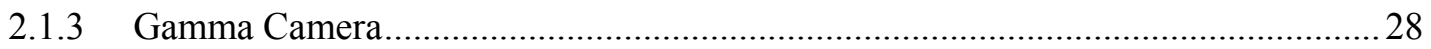

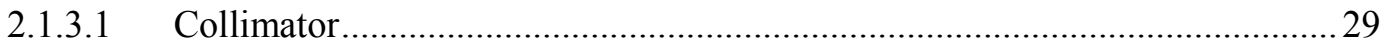

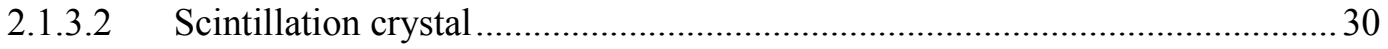

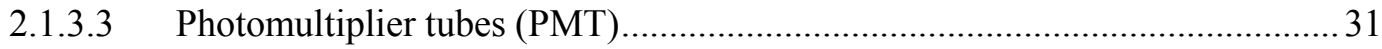

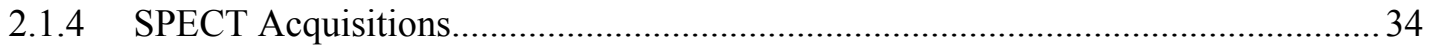

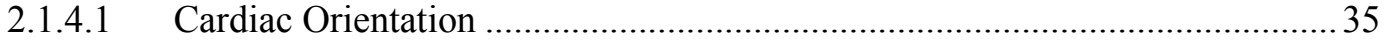

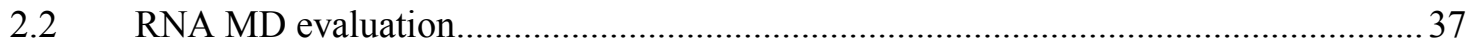

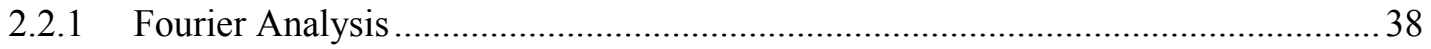

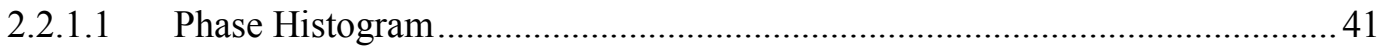

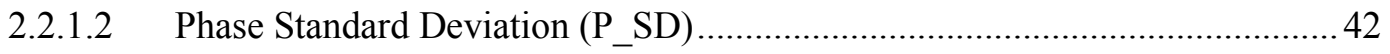

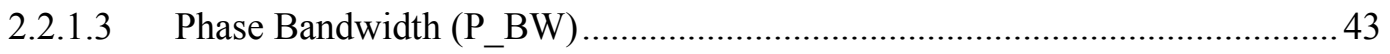

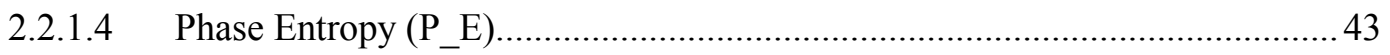

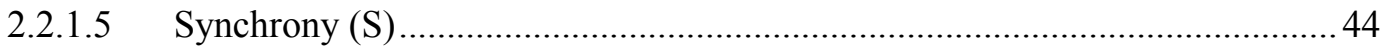

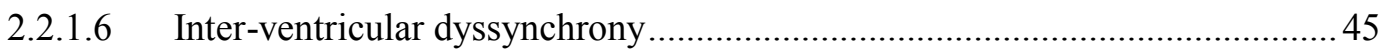

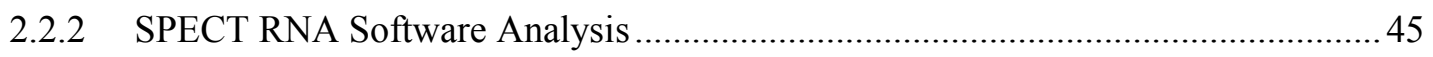

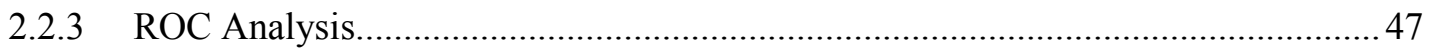

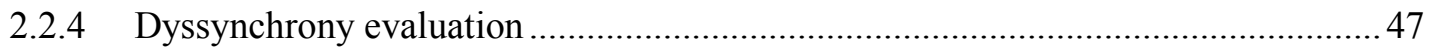

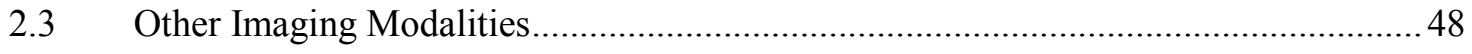

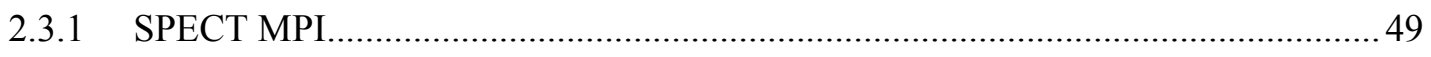

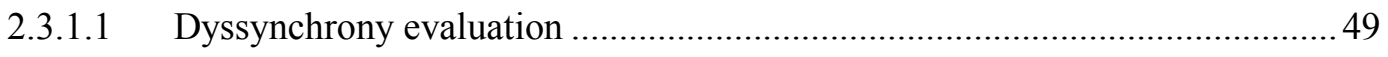

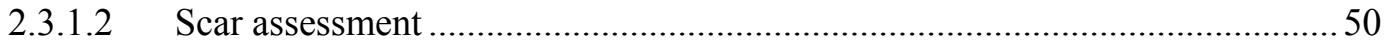

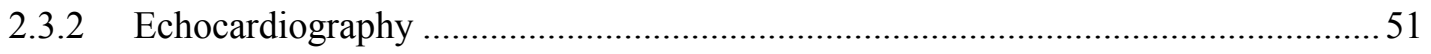

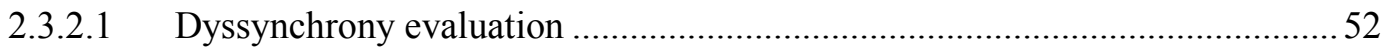

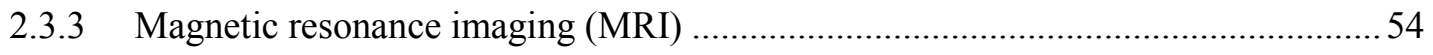

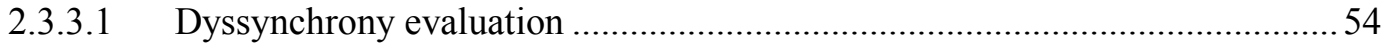




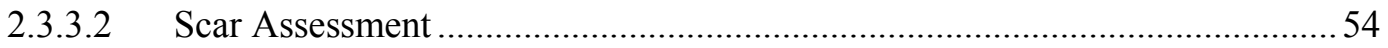

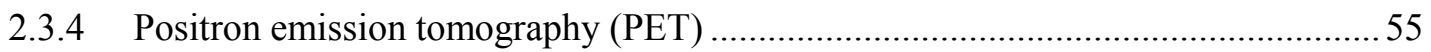

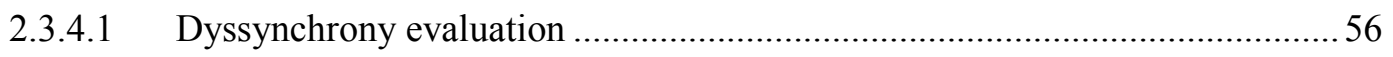

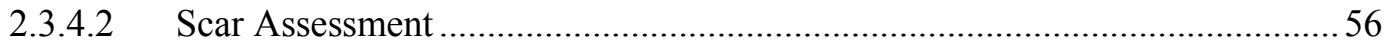

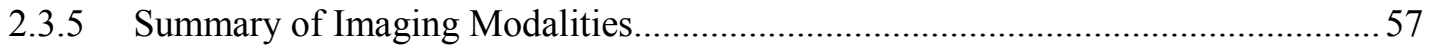

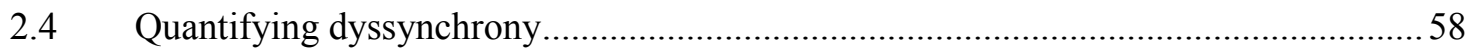

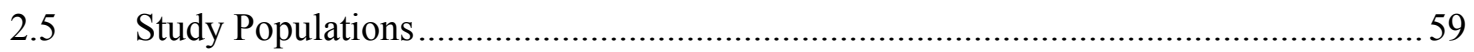

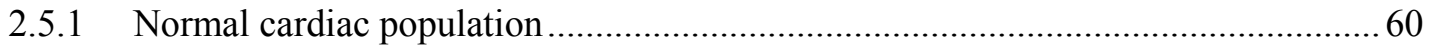

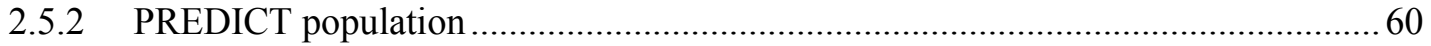

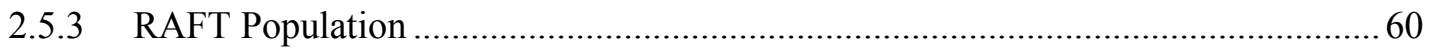

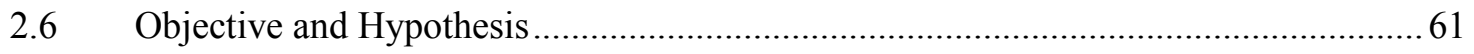

Chapter 3 Evaluation of the correlation between scar and amplitude ..................... 62

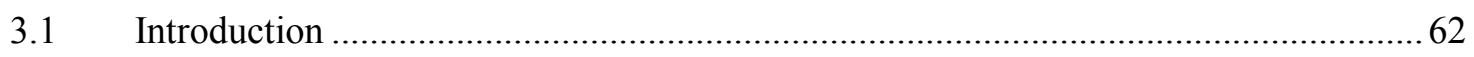

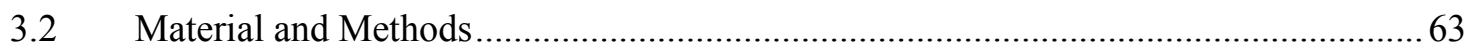

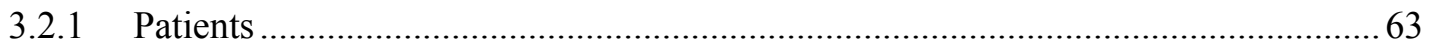

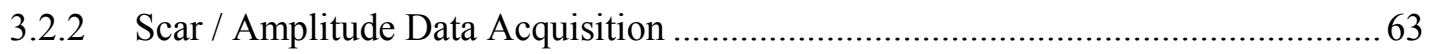

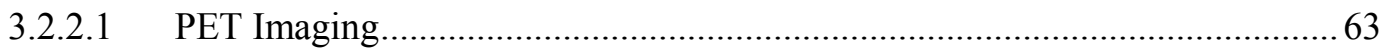

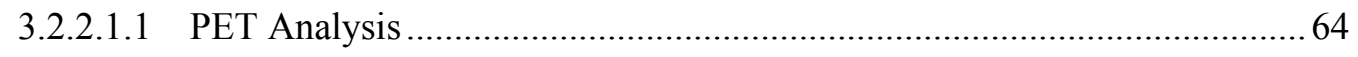

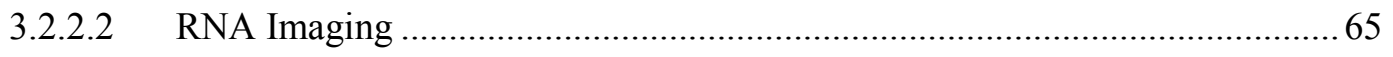

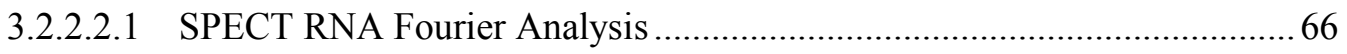

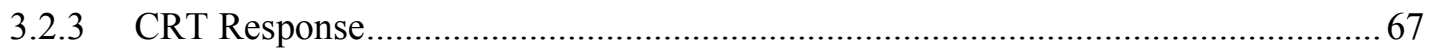

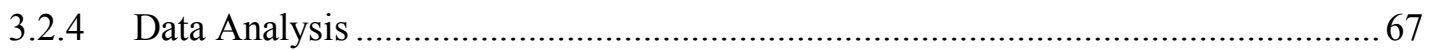

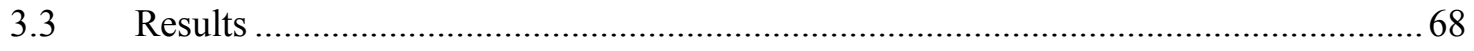

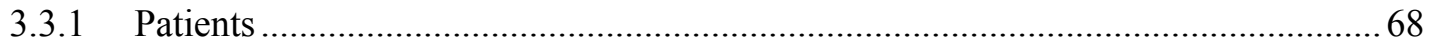

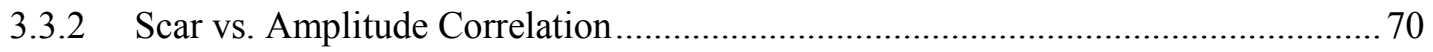

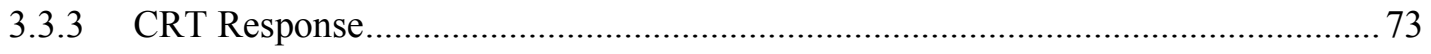




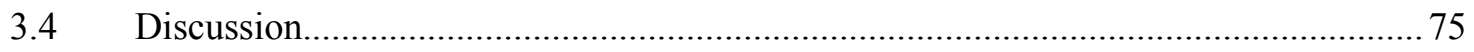

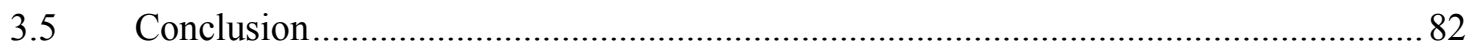

Chapter 4 Development of novel FA parameters...................................................... 83

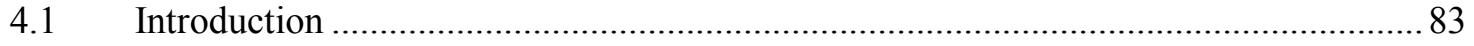

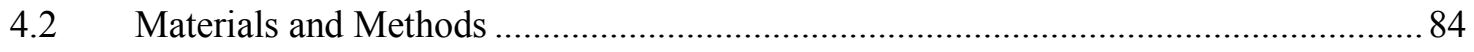

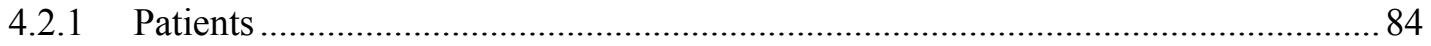

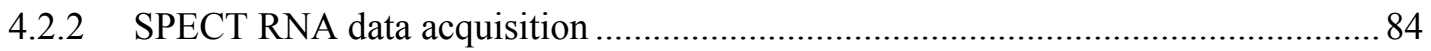

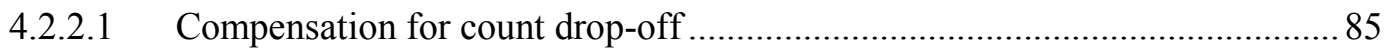

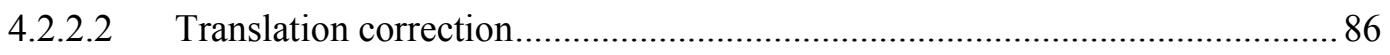

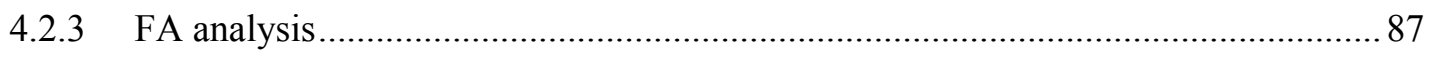

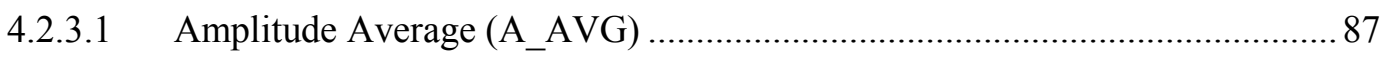

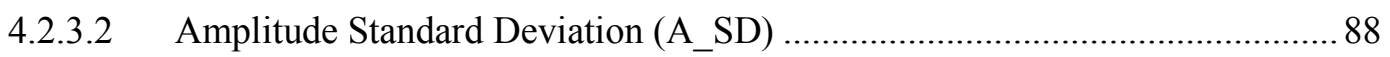

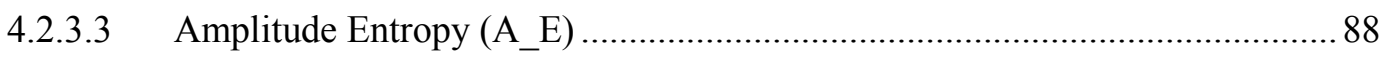

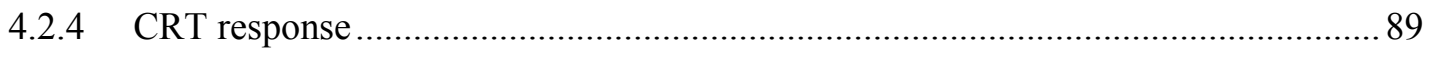

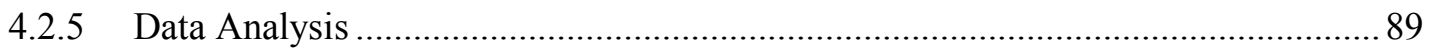

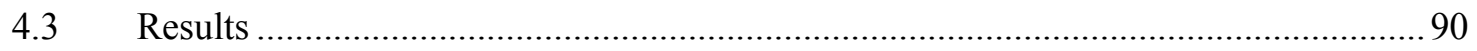

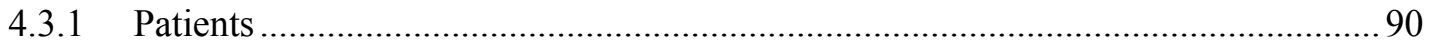

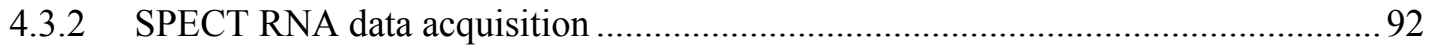

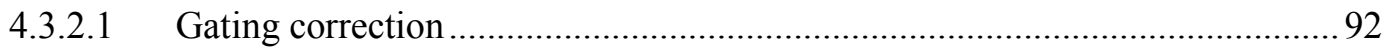

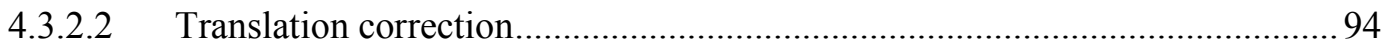

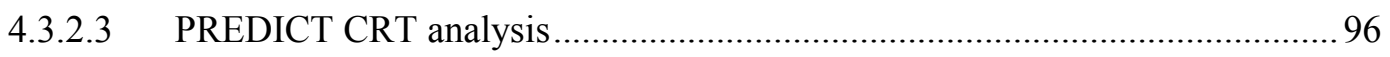

4.3.2.3.1 Impact of Translation Correction on prediction of CRT response .............97

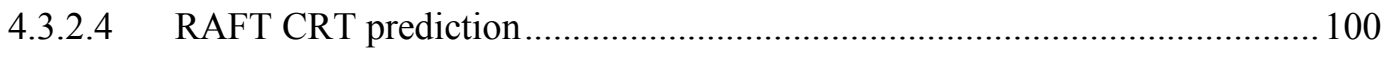

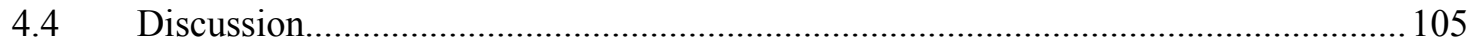

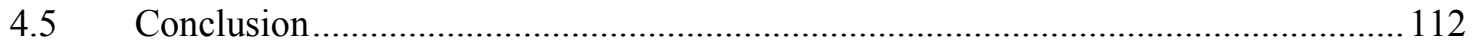


Chapter 5 Development of cluster analysis

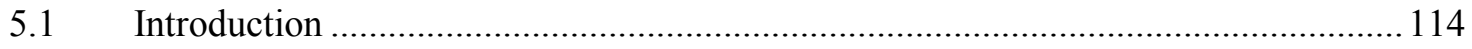

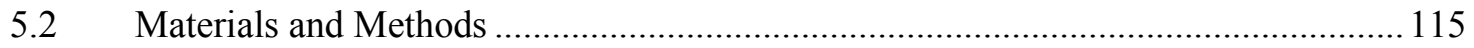

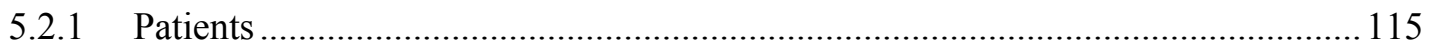

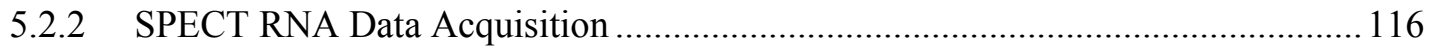

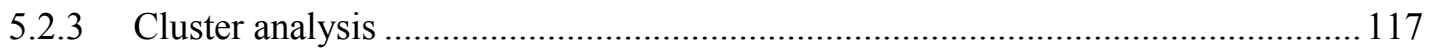

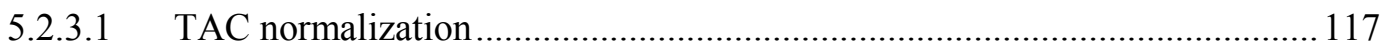

5.2.3.2 Development of a normal subject database ............................................... 118

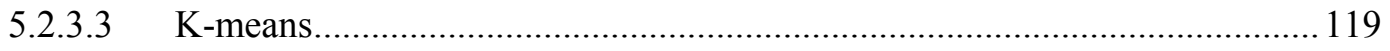

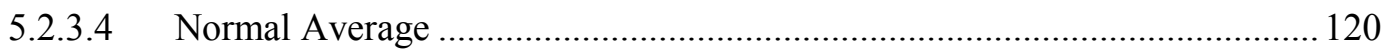

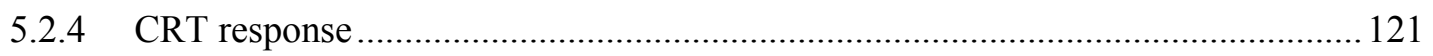

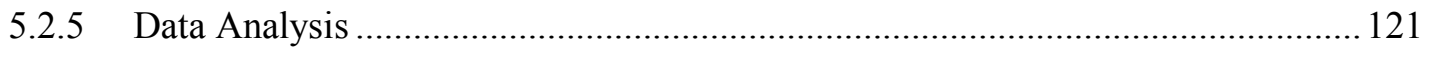

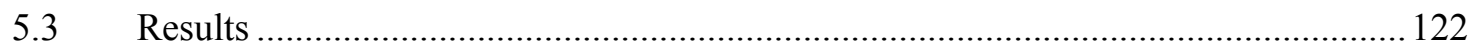

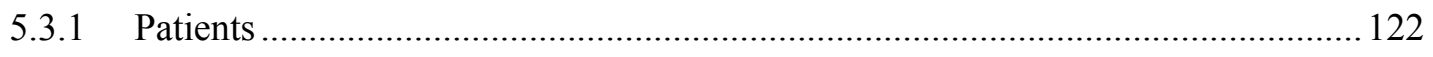

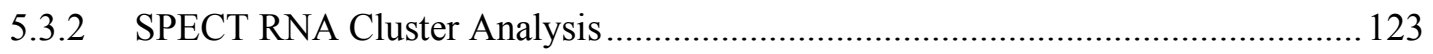

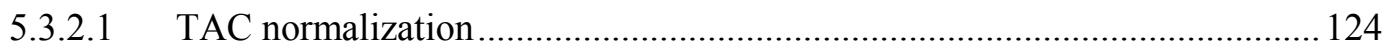

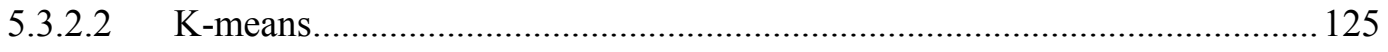

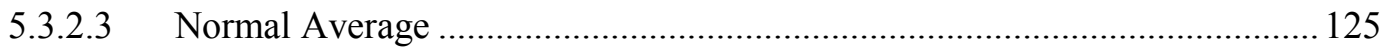

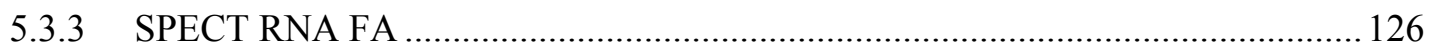

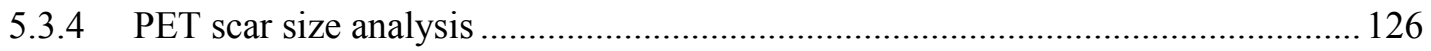

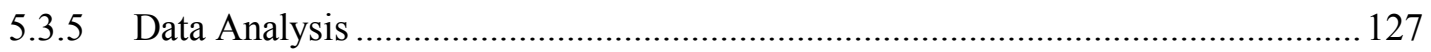

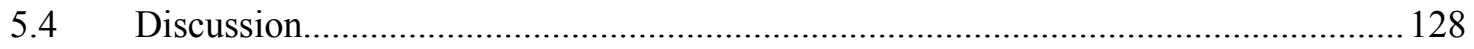

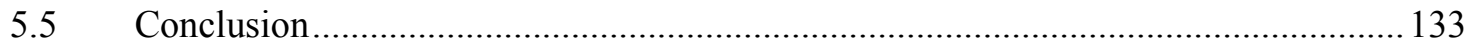

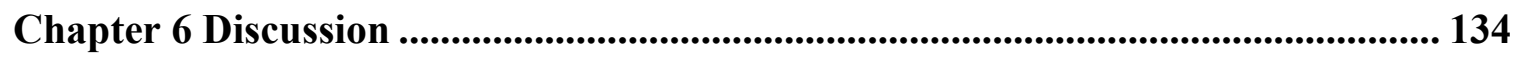

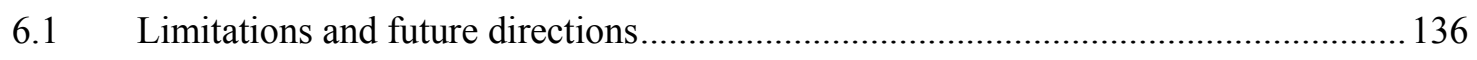

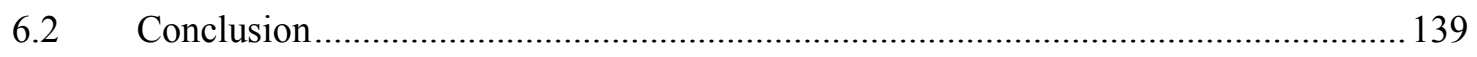


Appendix A Copyright Permission.................................................................................. 140

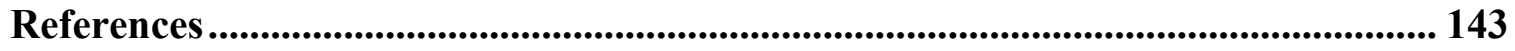




\section{List of Tables}

Table 1.1: Summary of the New York Heart Association heart failure classification ..... 13

Table 2.1: Properties of common radionuclides used in SPECT imaging....................... 25

Table 3.1 Patient demographics for the PREDICT population stratified by responder

status

Table 3.2: Patient demographics stratified by ischemic etiology and responder status.... 70

Table 3.3: Correlation coefficient between AmpSize and scar size in the lateral wall .... 71

Table 3.4: Correlation coefficient between AmpScore and scar score in the lateral wall 72

Table 3.5: Lateral wall ROC area under the curve values for CRT response prediction for AmpSize, AmpScore, scar size and scar score for each group.................................... 74

Table 4.1: Patient demographics for the RAFT ICD+CRT population stratified by

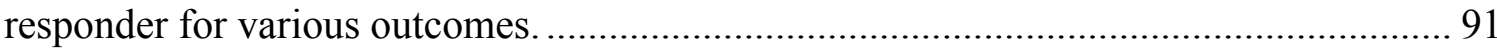

Table 4.2: Impact of gating artifacts corrections on FA parameters............................. 93

Table 4.3: Impact of translation correction on FA parameters …................................ 95

Table 4.4: Septal wall FA parameters differences between responders and non-responders

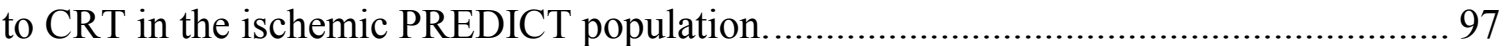

Table 4.5: p-values of various septal wall FA parameters between CRT responders and

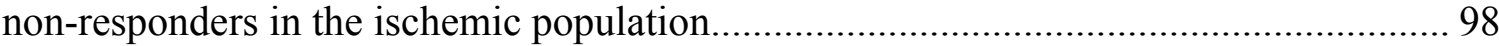


Table 4.6: ROC AUC values for the PREDICT ischemic population using the best

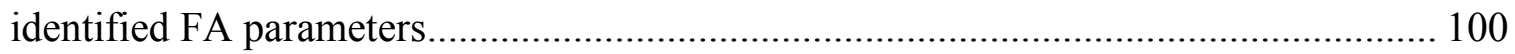

Table 4.7: Comparison of pre-selected FA parameters means between responders and non-responders to CRT in the RAFT ICD and CRT population............................... 101 Table 4.8: Comparison of responder septal A_AVG and septal A_SD values in the ICD and ICD+CRT RAFT population using death as non-response criteria. 103 Table 4.9: ROC AUC values for the RAFT ICD+CRT population using the best identified FA parameters. 105

Table 5.1: Corresponding score per number of outlier data points in a TAC for each cluster group.

Table 5.2: Patient demographics for the normal population, CRT responder and nonresponder groups. 123

Table 5.3: Intra- and inter- subject variability of the 3 different normalization techniques 124

Table 5.4: Global and segmental ROC AUC values for all populations using the K-means algorithm 125

Table 5.5: Best cluster analysis ROC AUC results for all populations. 126 


\section{List of Figures}

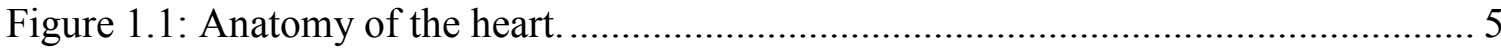

Figure 1.2: Sample electrocardiogram with waveforms $P$ to $T$ labeled.............................. 6

Figure 1.3: Anatomy of the conduction system of the heart........................................... 8

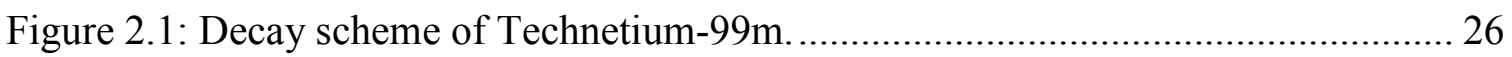

Figure 2.2: Schematic diagram of an Anger camera........................................................ 29

Figure 2.3: Sample R-R wave electrocardiogram divided into 8 time bins....................... 34

Figure 2.4: SPECT RNA reconstructed image at a) SA view, b) HLA view and c) VLA view at an arbitrary slice. d) A schematic view of the HLA orientation along with the

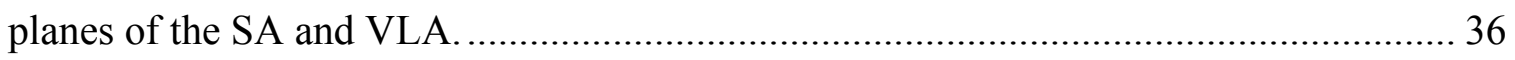

Figure 2.5: Sample 5-segment polar map representing a two dimensional view of the left ventricle, from apex (middle) to the basal slices (outer rings). ....................................... 37

Figure 2.6: Sample first order Fourier fit of actual measured counts ............................... 40

Figure 2.7: Sample phase histogram of a) a normal cardiac patient and b) a dyssynchrony

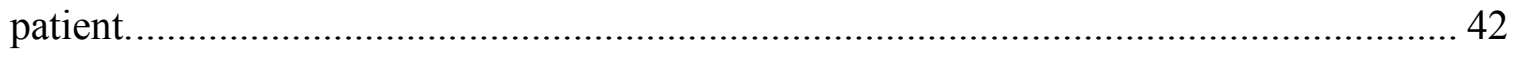

Figure 3.1: Correlation between lateral AmpSize and lateral wall scar size for: A) ischemic responders, B) ischemic non-responders, C) non-ischemic responders and D)

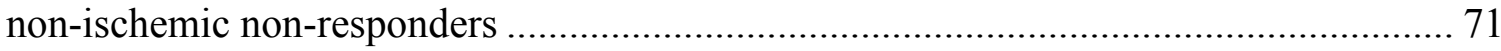


Figure 3.2: Correlation between lateral AmpScore and lateral wall scar score for: A) ischemic responders, B) ischemic non-responders, C) non-ischemic responders and D) non-ischemic non-responders

Figure 3.3: ROC Curves for a) Scar size and AmpSize and b) Scar Score and AmpScore in the lateral wall for the ischemic population 75

Figure 4.1: Normalized counts as a function of gate for a normal curve (green), a mildly abnormal curve requiring rescaling (yellow) and a severely abnormal curve requiring a drop gate and rescaling (red). 92

Figure 4.2: Average SA translation in the $\mathrm{x}$-axis (top) and $\mathrm{y}$-axis (bottom) as a function of gate number after applying translation correction

Figure 4.3: Change in septal and lateral wall a) S and P_SD and b) A_AVG and A_SD, after applying translation correction

Figure 4.4: Typical change in TAC shape following translation correction in the a) septal wall and b) lateral wall. 96

Figure 4.5: ROC curves for the PREDICT ischemic population for a) septal P_E, b)

Septal S, c) septal A_AVG and d) septal A_SD.

Figure 4.6: ROC curves for the RAFT ICD+CRT population for a) septal A_AVG, b) Septal A_SD using death as non-response criteria and, c) global S and d) global P_SD using CHF hospitalization as non-response criteria....... 104

Figure 5.1: Two sample unnormalized TACs (left) are each normalized to their own average (dashed line) to produce two individually normalized curves (right). 118 Figure 5.2: Inter- and intra-subject variability at each gate for the 3 different normalization techniques. 
Figure 5.3: Comparison of ROC curves for the best results from cluster analysis, FA

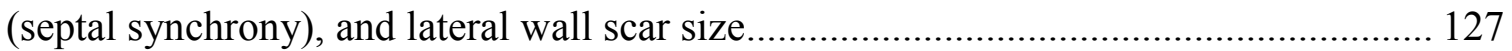




\section{List of Acronyms}

$\begin{array}{ll}\text { A } & \text { Amplitude } \\ \text { ACC } & \text { American College of Cardiology } \\ \text { AHA } & \text { American Heart Association } \\ \text { AUC } & \text { Area Under the Curve } \\ \text { AVG } & \text { Amplitude Average } \\ \text { BW } & \text { Bandwidth } \\ \text { CCS } & \text { Canadian Cardiovascular Society } \\ \text { CHF } & \text { Congestive Heart Failure } \\ \text { COM } & \text { Centre of Mass } \\ \text { CRT } & \text { Cardiac Resynchronization Therapy } \\ \text { E } & \text { Entropy } \\ \text { ECG } & \text { Electrocardiogram } \\ \text { ED } & \text { Electrical Dyssynchrony } \\ \text { EF } & \text { Ejection Fraction } \\ \text { EDV } & \text { End Diastolic Volume } \\ \text { ESV } & \text { End Systolic Volume } \\ \text { FA } & \text { Fourier Analysis } \\ \text { HLA } & \text { Horizontal Long Axis } \\ \text { IC } & \text { Internal Conversion } \\ \text { ICD } & \text { Implantable Cardioverter Defibrillator } \\ \text { ICD-10 } & \text { International Classification of Disease Version 10 } \\ \text { LBBB } & \text { Left Bundle Branch Block } \\ \text { LA } & \text { Left Atrium } \\ \text { LOE } & \text { Level of Evidence } \\ & \end{array}$




\begin{tabular}{ll} 
LV & Left Ventricle \\
MD & Mechanical Dyssynchrony \\
MPI & Myocardial Perfusion Imaging \\
MRI & Magnetic Resonance Imaging \\
NYHA & New York Heart Association \\
OMT & Optimal Medical Therapy \\
P & Phase \\
PET & Positron Emission Tomography \\
PMT & Photomultiplier Tubes \\
QOL & Quality of Life \\
RA & Right Atrium \\
RBBB & Right Bundle Branch Block \\
RBC & Red Blood Cells \\
RNA & Radionuclide Angiography \\
ROC & Receiver Operating Characteristic \\
ROI & Region of Interest \\
RV & Right Ventricle \\
S & Synchrony \\
SA & Short Axis \\
SD & Standard Deviation \\
SPECT & Single Photon Emission Computed Tomography \\
TA & Time Activity \\
TAC & Time Activity Curve \\
TD & Time Distance \\
TDI & Tissue Doppler Imaging \\
UOHI & University of Ottawa Heart Institute \\
VLA & Vertical Long Axis \\
& \\
\hline
\end{tabular}




\section{Overview}

Mechanical dyssynchrony (MD) reduces the heart's pumping efficiency and is a common occurrence in congestive heart failure (CHF). One of the existing treatments for CHF is cardiac resynchronization therapy $(\mathrm{CRT})$ in which a pacemaker is implanted into a patient in an attempt to resynchronize the patient's heart, thus reducing MD. However, CRT has been shown to provide no clinical benefit in a large proportion of recipients. Currently, MD is not used as a patient selection criterion for CRT despite evidence that links CRT response to improvements in MD. The development of novel methodologies (and improvement of existing ones) to characterize and quantify baseline MD could help improve CRT response rates.

In this thesis, new methodologies are developed and validated using single photon emission computed tomography (SPECT) radionuclide angiography (RNA) to improve wall motion analysis which may lead to improved quantification of MD. The ability of the MD parameters to predict outcome to CRT is also investigated. Chapters 1 and 2 cover a broad range of introductory material, beginning in chapter 1 with information on dyssynchrony, CHF, CRT, the problem of non-response and how they are all related. In Chapter 2, a review of SPECT physics is given. A literature review of what various imaging modalities, including SPECT RNA, have done to measure dyssynchrony and 
predict outcome of CRT follows. New SPECT methodologies are then broadly discussed.

Chapter 3 will discuss the possible correlation that exists between amplitude and scar values obtained from PET and compare their ability to predict response to CRT. Chapter 4 will evaluate the potential of novel Fourier analysis amplitude-based parameters to predict CRT outcome in a sample population, and further evaluate the ability to predict response in a large double blinded study population. Chapter 5 will show the development of cluster analysis methodologies and evaluate how well they can predict response to CRT using a small sample population. Finally, a discussion on the results of the work presented in this thesis is given in chapter 6 along with final conclusions.

The work presented in this thesis provides several contributions relevant to the topic of cardiac wall-motion analysis and prediction of CRT response. LV scarring has been previously identified as a key factor in predicting CRT response. It is shown that amplitude-based metrics correlate with those based on scar and, therefore, that wallmotion amplitude may be useful in predicting CRT response. Novel amplitude-based Fourier analysis parameters are developed and shown to be predictive of CRT response in two different clinical studies. A novel methodology called cluster analysis, is developed and optimized for wall-motion analysis and shown to perform at least as well as Fourier analysis approaches to CRT prediction. Finally, it is shown that segmental analysis, based on a 5-segment heart model, can detect significant effects that are diluted and not significant with global analysis of the same parameters. Current practice is to only 
evaluate global parameters and this suggests a new direction that can be pursued and that particular attention should be paid to the septal wall for the prediction of CRT response. 


\section{Chapter 1}

\section{Medical Background and Motivation}

\subsection{Cardiac Anatomy and physiology}

\subsubsection{Cardiac cycle}

The human heart is comprised of 4 chambers: the left (LA) and right atrium (RA) (for a complete list of acronyms, see page $\mathrm{xx}$ ), as well as the left (LV) and right ventricle (RV), as seen in Figure 1.1. The LV is the largest chamber and the strongest muscle of the heart and pumps blood to the body. As deoxygenated blood returns from the body to the heart, it is directed into the right atrium by the superior and inferior vena cava. Blood then flows passively into the RV during diastole. Near the end of diastole, the RA contracts, forcing additional blood into the RV. A subsequent contraction of the RV, during systole, pumps the blood through the pulmonary valve, the pulmonary arteries and towards the lungs for oxygen absorption. The newly oxygenated blood then returns to the heart via the pulmonary veins, entering in the LA. During diastole, the pressure difference between LA and LV increases, the mitral valve opens and blood flows passively into the LV. Near the end of diastole, the LA contracts, forcing additional blood into the LV. Finally, at systole, the LV contracts and the pressure difference between the 
LV and the arteries increases until the aortic semilunar valve opens and blood is ejected through the aorta and circulated through the entire body, including the heart muscle itself.

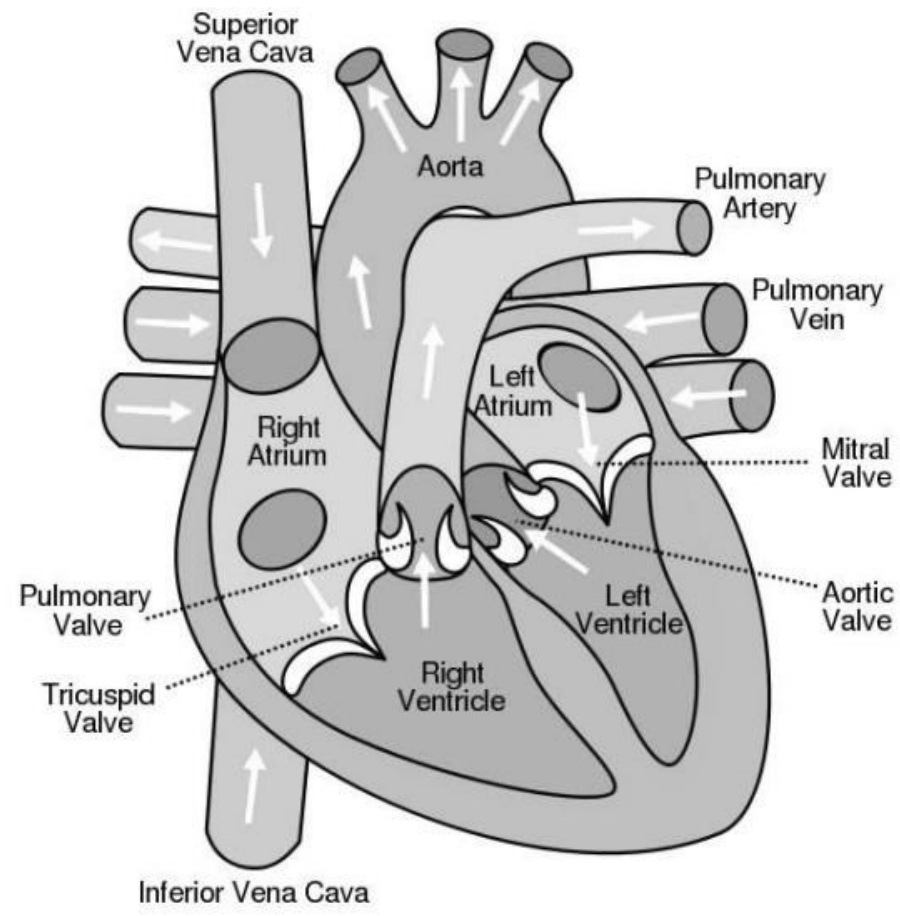

Figure 1.1: Anatomy of the heart outlining the major chambers, veins, arteries and valves. Image obtained from http://www.sciencekids.co.nz/ (4) (public domain).

Based on the cardiac cycle, three important functional cardiac parameters are end-systolic volume (ESV), end-diastolic volume (EDV) and ejection fraction (EF). LV ESV and EDV, typically expressed in milliliters, represent the volume of blood present in the left ventricle at systole and diastole respectively. ${ }^{*} \mathrm{EF}$ is the percent of the maximum blood volume that is ejected from the left (LVEF) or right (RVEF) ventricle during a cardiac cycle and is calculated using equation 1.1 below. The LVEF value of a healthy heart is greater than $50 \%$ whereas a LVEF less than $40 \%$ is considered to be impaired (5).

\footnotetext{
* Volumes for the right ventricle are also measured and may be indicated as RVESV and RVEDV to distinguish them from the LV values
} 


$$
E F(\%)=100 * \frac{E D V-E S V}{E D V}
$$

\subsubsection{Electrophysiology}

An electrocardiogram (ECG) is a graphical representation of the potential changes, due to depolarization of the heart, occurring over time as the heart beats. In order to view the potential changes, several electrodes are strategically positioned on a patient and connected to a monitor. An ECG is an extremely useful diagnostic tool because there are several characteristic waveforms, segments and intervals that can all be quantified and compared to a healthy heart pattern in normal sinus rhythm. Waveforms on an ECG are labeled alphabetically from $\mathrm{P}$ to $\mathrm{U}$ and prolonged intervals and irregularly shaped waves are indicators of conduction problems. The anatomical location of a conduction problem can often be pinpointed from the irregular ECG segment. An ECG can be used to assess a variety of medical issues, which include but are not limited to: conduction disturbances, injury or disease, artificial pacemaker function, ischemic damage and response to medication (5). A sample ECG is shown in Figure 1.2.

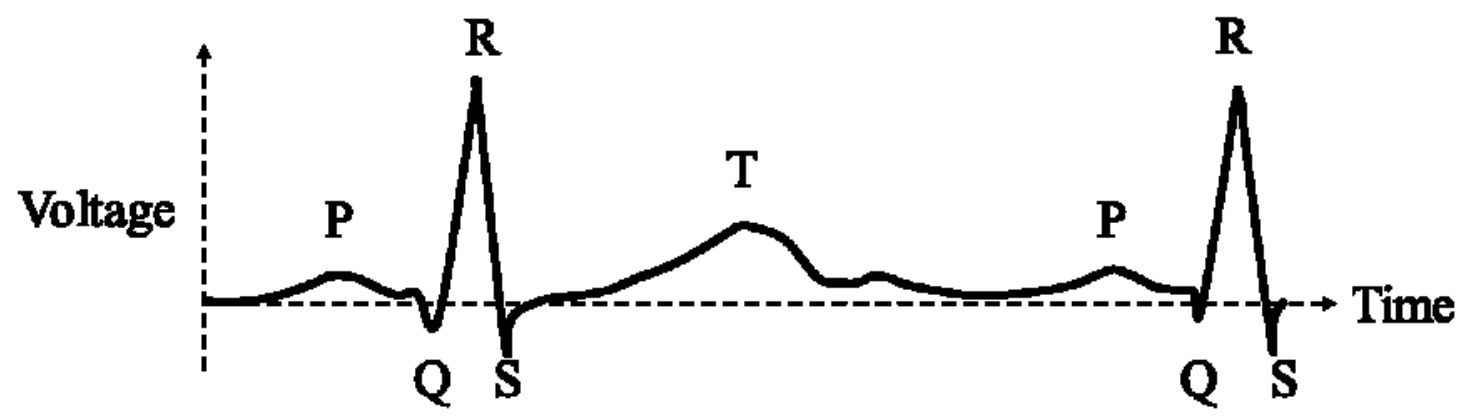

Figure 1.2: Sample electrocardiogram with waveforms $P$ to $T$ labeled 
The intrinsic conduction system of the heart is an electrical system that is responsible for ensuring a rhythmic contraction of the heart as well as providing a rapid conduction path for the electrical impulses generated. The cells in the intrinsic conduction system are called pacemaker cells and have the ability to conduct a signal rapidly. The remaining cardiac cells are called myocardial cells and possess the ability to contract following excitation. When there is no electrical activity present, the natural resting state of the cells is polarized. The physical path undertaken by an electrical impulse is summarized below and in Figure 1.3.

The heart's natural pacemaker, the sinoatrial node, is responsible for generating a rhythmic electrical signal by initiating an electrical impulse which will propagate through the heart muscle following specific conduction channels. As the impulse circulates, it causes nearby myocardial cells to depolarize, leading to contraction. A short time after, the cells repolarize as they relax and return to their natural resting state. After leaving the sinoatrial node, the impulse progresses through conduction channels in the atria, causing contraction in both the LA and RA. This is observed as a small bump on an ECG and is labeled as the $\mathrm{P}$ wave. The impulse then reaches the atrioventricular node where it branches off through the atrioventricular bundle into the left and right bundle branches which run along septum down to the apex and back up the lateral side of the left and right ventricles respectively. Along the bundle branches are several Purkinje fibers which further branch off onto the ventricular surfaces. As the impulse passes through all these channels, the ventricles depolarize (while the atria repolarize) and a large spike on the 
ECG (QRS complex) is observed. In normal sinus rhythm, the QRS duration is between 60 and $100 \mathrm{~ms}$. Finally, a $\mathrm{T}$ wave is observed as the ventricles repolarize a short time after. This entire process typically occurs in less than 1 second and in a healthy heart, the relative timing of contraction from the site of earliest activation to the site of latest activation within a ventricle is relatively homogeneous.

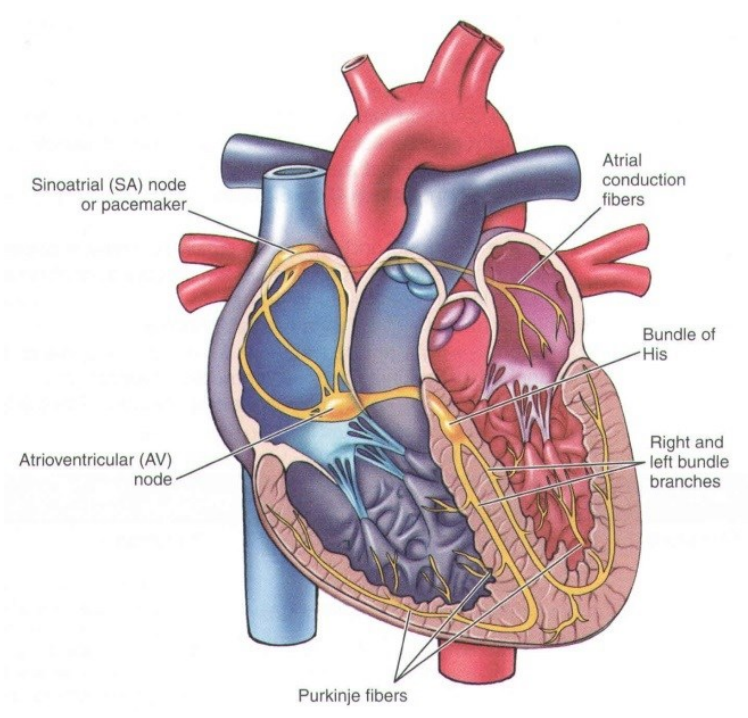

Figure 1.3: Anatomy of the conduction system of the heart. Image obtained from Aehlert (5) ${ }^{\dagger}$

\subsection{Ventricular Dyssynchrony}

In a normal heart, at systole, both ventricles contract simultaneously, (in synchrony). Inter-ventricular dyssynchrony occurs when there is a delay between the contractions of the two ventricles. Furthermore, in order to pump blood efficiently, the relative timing of contraction of various myocardial wall segments within a single ventricle must be synchronous. In certain cases, this synchrony is lost and is called intra-ventricular

\footnotetext{
${ }^{\dagger}$ Permission was granted to reprint this figure first published in ACLS Study Guide, $3^{\text {rd }}$ Ed., Aehlert, B., Title of article, Page 127, Copyright Mosby Jems Elsevier (2007). See Appendix A.
} 
dyssynchrony. It is the measurement of intra-ventricular dyssynchrony that is the focus of this thesis.

\subsubsection{Electrical vs. mechanical dyssynchrony}

During the conduction of the electrical impulse that stimulates contraction, it is possible that the signal is delayed or blocked from its typical conduction path. The impulse is then forced to propagate through 'slower' tissues, such as muscle cells, which can cause segments of a ventricle 'downstream' of the impulse to receive a delayed signal. On an ECG, the QRS complex is observed as the ventricles depolarize and contract. A broad QRS duration is a marker of electrical dyssynchrony (ED) as it indicates that the impulse is taking a longer time to reach certain segments of a ventricle. A QRS duration of 120 ms or greater is considered wide or abnormal. Thus, ED signifies a delay in the electrical impulse as it travels through the conduction system.

ED can be caused by left bundle branch block (LBBB), which is a condition in which the electrical signal is blocked or delayed in the left bundle branch. This condition is readily identified on an ECG and can be diagnosed using the American Heart Association (AHA) and American College of Cardiology (ACC) recommendations for the standardization and interpretation of LBBB (6), where one of the major criteria is a QRS duration greater than 120 ms. A blockage will cause inter-ventricular dyssynchrony as the LV signal propagation is delayed with respect to the RV signal. It can also cause intra-ventricular dyssynchrony if some segments of the LV were activated prior to the blockage. Under a normal conduction system, the electrical impulse reaches the LV lateral wall last and any 
blockage in the left bundle branch often leads to delayed lateral wall contraction. A blockage in the right bundle branch is also possible and is known as right bundle branch block (RBBB) and causes similar delays but in the RV.

Mechanical dyssynchrony (MD) represents the relative timing delay in the actual physical contraction of the ventricles and may refer to either inter- or intra-ventricular dyssynchrony, or both. Currently, there exists no gold standard measure of mechanical dyssynchrony, but there have been several metrics developed for the purpose of measuring $\mathrm{MD}$, using various imaging modalities. These are discussed further in chapter 2.

It is logical to conclude that the presence of $\mathrm{ED}$ will cause $\mathrm{MD}$, as an electrical delay can cause a delay in the timing of physical contraction of the segment with respect to other segments. However, studies have shown that this is not always the case as some patients with ED do not have significant MD (and vice versa). One condition does not necessarily lead to the other (7-9). A possible reason suggested by Kass (10) is that QRS duration takes into account the depolarization of both ventricles, while delayed physical contraction may be localized to part of a single ventricle. The author also indicates that delayed signal propagation in the LV could be offset by rapid signal propagation in the $\mathrm{RV}$, producing a normal QRS even though significant MD is present in the LV (10).

The presence of inter-ventricular dyssynchrony impacts the heart's physiology. For example, in an LBBB patient, the delayed onset of LV contraction causes RV diastole 
and systole to precede that of the LV (11). The increase in RV pressure during contraction can reverse the typical transseptal pressure and cause movement of the interventricular septum into the $\mathrm{LV}$, which will reduce the contribution of the septum to ejecting blood during LV systole $(11,12)$.

Intra-ventricular dyssynchrony also negatively impacts the heart's physiology. The uncoordinated contraction of various wall segments results in inefficient pumping. The ventricular pressure is low when the early segments contract resulting in relatively little blood ejection. Conversely, the areas of late contraction are overburdened as they compensate for the early wasted work. The end result is a reduction in ejection fraction and an increase in ESV as less blood exits the LV during contraction $(12,13)$. A study by Ghio et al. reported that no significant relation existed between inter- and intraventricular dyssynchrony (14).

\subsection{Congestive Heart Failure (CHF)}

Congestive heart failure is a serious medical condition in which the heart's pumping efficiency is severely reduced and therefore it is unable to circulate enough blood to meet demand of the body. As discussed in the previous section, the presence of a significant degree of dyssynchrony weakens the heart, and can lead to CHF.

Heart failure can occur in either the LV, RV, or both. When there is substantial dyssynchrony present, the heart attempts to regulate cardiac output and compensate for the poor pumping efficiency by modifying factors such as heart rate and stroke volume. 
However, over time, this will weaken the heart further and the increased stress of onesided heart failure often leads to heart failure of the other ventricle. In certain cases, to compensate for the lack of blood volume being ejected, the ventricle will gradually increase in size so that a larger volume of blood is pumped out for a constant EF. However, this dilation of the ventricle, known as dilated cardiomyopathy, weakens the heart muscle further, eventually worsening the CHF.

In LV heart failure, blood is not ejected from the LV efficiently and gets backed up in the pulmonary veins and the lung blood vessels. This leads to swelling and leaking of fluid into the lungs, causing pulmonary edema $(5,15)$. In RV heart failure, poor RV pumping causes blood to back up in the body's venous system. This leads to fluid congestion and swelling in the vascular system as well as in the extremities, such as ankles, feet and fingers $(5,15)$. Other common symptoms of CHF are fatigue and shortness of breath.

A common chain of $\mathrm{CHF}$ incidence is that atherosclerosis (formation of plaque in coronary arteries) leads to coronary heart disease. As plaque builds up, the artery narrows, reducing blood flow to the myocardium. The chronic lack of oxygen supply (ischemia) may cause impaired contractile function. A patient may also suffer a myocardial infarction if a plaque ruptures and occludes an artery. This acute event causes a lack of oxygen downstream of the occlusion and can lead to the death of myocardial tissue which, if blood supply is not recovered rapidly enough, is permanent. Consequently, scar tissue is formed; the heart weakens further and becomes impaired as the scar tissue loses contractile function. Hibernating tissue may also be present in the 
myocardium and is similar to scar tissue except that the functionality and contractile function of the hibernating tissue may be restored. The loss of viable tissue leads to dyssynchrony, as previously described, and a lowered pumping efficiency, causing heart failure.

In order to better categorize the severity of $\mathrm{CHF}$ and facilitate patient classification, the New York Heart Association (NYHA) (16) established four stages of CHF. A table summarizing the four different stages of the NYHA is provided below.

Table 1.1: Summary of the New York Heart Association heart failure classification (16)

NYHA Class Symptoms

\begin{tabular}{ll}
\hline I & No exercise limitations. Ordinary activity does not cause fatigue or \\
& pain. (No symptoms) \\
II & Slight exercise limitations. Activity results in fatigue or chest pain. \\
III & Significant exercise limitation. Less than ordinary activity causes \\
& fatigue or chest pain \\
IV & Unable to carry out exercise or activity without significant discomfort. \\
& Symptoms may occur at rest
\end{tabular}
$\overline{\mathrm{NYHA}}=$ New York Heart Association

Based on the World Health Organization's International Classification of Diseases version 10 (ICD-10), CHF (ICD-10 code I50) is one of the leading causes, behind only ischemic disease, for both hospitalization and mortality among cardiovascular diseases (17). In Canada, the latest data indicates that $2.2 \%$ of all hospitalizations classified CHF 
as the primary diagnosis (2005-2006), and that $1.8 \%$ of all deaths were due to CHF (2009) (18).

CHF hospitalization rates increase significantly in age groups above 65 years, considerably later than for ischemic heart disease hospitalizations which increase rapidly in patients in age groups over 45 (17). This supports the notion that ischemic heart disease is present years before congestive heart failure occurs.

Overall mortality rates for CHF have been decreasing since the year 2000 but not as rapidly as other cardiovascular diseases, such as ischemic heart disease which have been in decline since the 1970's (17). This may be explained by the improved survival and management of ischemic heart disease and myocardial infarction over the years which have led to increased incidence of $\mathrm{CHF}$ as an end-stage outcome. The recent decline of CHF mortality may be attributed to improved treatments such as optimal medical therapy and cardiac resynchronization therapy.

\subsubsection{Cardiac Resynchronization Therapy (CRT)}

CRT is a surgical procedure in which an artificial pacemaker is implanted into a patient in an attempt to resynchronize the patient's heart. A pacemaker is a sophisticated small battery powered device capable of sending out a current of electricity through the leads attached to it. The latest generation of pacemakers possesses three wires which are strategically attached to the LA, RV and LV respectively. The lead attached to the LA senses when the heart's sinoatrial node generates an impulse and relays the timing 
information to the pacemaker. Two separate electrical signals are then sent through the other two leads following pre-programmed delays, which will initiate contraction of myocardial tissue fused to the lead. The signal will then spread from that location following regular conduction channels. By sending artificial signals, the pacemaker is able to reduce the timing delays between (and within) the ventricles, caused by blockages or other conduction abnormalities. CRT aims to improve on the timing of the physical contraction, thus reducing $\mathrm{MD}$, but has also been shown to reduce $\operatorname{ED}(1,19)$.

CRT was officially approved by the United States Food and Drug Administration in 2001 but has been used since the 1990's (12). CRT has been investigated in various CHF populations to determine whether it produces clinical benefit and a summary of the major CRT trials is given below.

The MUSTIC (20) and MIRACLE (19) trials conducted in the early 2000's demonstrated that patients with moderate to severe CHF (LVEF $<35 \%$, QRS $>130 \mathrm{~ms}$, NYHA class III/IV, 6 minute walk test $<450 \mathrm{~m}$ ) receiving CRT showed an improvement in quality of life and exercise capabilities within a 6 month follow-up period. These findings were confirmed by another large multicentre trial, CARE-HF $(21,22)$, who demonstrated that CRT significantly improved quality of life, and also restored LV function and survival in moderate to severe CHF populations. These studies provide substantial proof of the clinical benefits of CRT in moderate to severe CHF patients. 
Further studies were designed to determine the benefits of CRT in less symptomatic patients (NYHA class I or II) as well as the impact of adding CRT to an implantable cardioverter defibrillator (ICD). The multicentre COMPANION study $(23,24)$ followed patients for $12+$ months to investigate, in a 3 -arm study, the effects of optimal medical therapy (OMT) alone, OMT $+\mathrm{CRT}$, and $\mathrm{OMT}+\mathrm{CRT}+\mathrm{ICD}$ on a population of moderate to severe CHF patients (LVEF $<35 \%$, QRS $>120 \mathrm{ms,} \mathrm{NYHA} \mathrm{class} \mathrm{III} \mathrm{or} \mathrm{IV).} \mathrm{The} \mathrm{study}$ demonstrated that CRT reduced mortality by $24 \%$ while CRT + ICD reduced the risk of death by $36 \%$, with respect to optimal medical therapy alone. In 2009 , results from the MADIT-CRT study $(25,26)$ found that in mild CHF patients (LVEF $<35 \%$, QRS $>130$ ms, NYHA class I or II), CRT+ICD significantly reduced the risk of future heart failure events in comparison to ICD alone. However, the overall risk of death was not significantly different between both groups. Finally, the RAFT study $(2,3)$, a large multicentre trial designed in Canada, investigated the benefits of adding CRT to ICD and OMT in mild to moderate CHF patients (LVEF $<30 \%$, QRS $>120 \mathrm{~ms}$, NYHA II or III). Published results of the RAFT study indicate there is a significant reduction in all-cause death as well as hospitalization for subsequent heart failure events. These major CRT studies provide conclusive evidence of the benefits of CRT in conjunction with an ICD in moderate to severe as well as in less symptomatic patients.

Following the results of these large trials, the AHA and ACC updated the guidelines for device-based therapies and appropriate use criteria. CRT for CHF patients received a class I recommendation in patients with LVEF $<35 \%$, sinus rhythm, LBBB, QRS $>150$ ms, following guideline directed medical therapy NYHA class II (level of evidence: B) or 
NYHA III/IV (LOE: A) $(27,28)$. Class IIa recommendations were established if the patient presented all the above criteria with exception of having a non-LBBB pattern (LOE: A) or a QRS duration between 120-149 ms (LOE: B) (27). Several other appropriate use criteria were established in the report and are not listed here.

Similarly, the Canadian Cardiovascular Society (CCS) recently published 8 recommendations on the use of CRT based on the evidence of the CRT trials (29). One major recommendation, categorized as strong recommendation, low-quality evidence, is that the patient selection for receiving CRT is: $\mathrm{LVEF}<35 \%$, QRS $>130 \mathrm{~ms}$, LBBB, NYHA class II-IV, sinus rhythm.

There is overwhelming evidence from large multicentre as well as smaller single studies that CRT improves quality of life, reduces mortality and hospitalization rates and enables reverse remodeling of the ventricle, re-establishing pumping efficiency, improving EF and reducing ESV in many CHF recipients $(2,19,20,22,24,26,30-32)$. However, several studies have shown that a minimum of $20 \%$, and possibly up to $50 \%$ of patients derive no benefit following CRT $(19,33-35)$. The elevated rate of non-response is of great concern not only to the patient for the risks and stress of surgery and whose condition may worsen without appropriate therapy, but also for the elevated costs associated with CRT.

The variation in non-response percentages can be partly explained by the different definitions of response used between studies. Non-response may be defined using hard clinical end-points (death, transplant), soft-clinical end-points (subsequent 
hospitalizations), LV remodeling measurements (improvement in LVEF and/or LVESV) and subjective capacity end-points (improvements in NYHA class or QOL score). Birnie et al. have summarized the various definitions of response explored in a variety of studies and remark that objective end-points are preferable to improve consistency between studies and minimize the placebo effect that subjective end-points might sustain (33). Hard clinical objective end-points are frequently observed in large multi-centre trials with sufficient statistical power and long follow-up times, such as the COMPANION (24), CARE-HF (22), RAFT $(2,3)$ and MADIT-CRT (26) which defined primary end-points either as death or subsequent hospitalization (all cause or cardiovascular). Yu et al. (36) conducted a study to investigate how short-term LV reverse remodeling and subjective capacity end points (NYHA class, QOL score and 6 minute walk test) relate to long-term prognosis following CRT. An LVESV reduction of $10 \%$ or more was found to be a strong predictor of long-term survival, while all subjective capacity end-points were not strong predictors (36).

There are several theories why the non-responder rate is elevated. Firstly, the current selection criteria for CRT, as outlined above, do not include any measure of MD despite evidence that MD is a key component of response $(37,38)$. QRS is a measure of ED, which often leads to MD, but MD may occur for other reasons and therefore a comprehensive evaluation (quantification) of MD is needed. Selection of patients based on an improved characterization and quantification of baseline mechanical dyssynchrony may help improve CRT response rates $(33,39-42)$. 
Secondly, the placement of the ventricular lead near the site of latest activation is thought to be crucial. Since CRT aims to reduce the timing delay in contraction, it is logical to position a pacemaker lead at the location of greatest delay (43). Following typical conduction systems, the greatest delay in the LV is often located in the posterolateral wall and less often in the septal wall $(44,45)$. Several studies have investigated the impact of the LV lead location and many have confirmed that LV MD and/or LV function improved the most with ideal lead placement at the site of latest activation $(33,44,46-49)$. Other studies have determined that the location of the RV lead did not significantly affect $\operatorname{LV} \operatorname{MD}(46,50)$.

Thirdly, the presence of scar tissue on the myocardium is associated with impaired wall motion. The rationale is that fibrotic scar tissue cannot be resynchronized since it is dead, and therefore no clinical benefit can arise from artificial pacing. Furthermore, placing a pacemaker lead directly at the site of latest activation would be impractical if that location is burdened with scar tissue. These are detailed further in section 2.2.

In summary, the heart is a complex organ with a unique dynamic interaction between the electrical and mechanical physiology. It is believed that MD plays a significant role in CHF and therefore, a way is needed to properly analyze and assess MD to better understand CHF. Despite not being included in current selection criteria for CRT, it is also believed that MD plays a significant role in CRT outcome. Non-invasive medical imaging modalities that can qualify and more importantly, quantify cardiac function are essential as they can help to determine cardiac abnormalities, scar, MD and the existing 
interaction between them and CRT. Various imaging modalities used to assess cardiac function in CHF patients are addressed in chapter 2. 


\section{Chapter 2}

\section{Imaging techniques for quantification of}

\section{dyssynchrony}

Medical imaging is believed to have a role to play in the treatment of CHF and the use of CRT. Non-invasive imaging can safely provide information on the quality of heart function, the presence of scar, hibernating tissues, contraction abnormalities and remodeling. It is not yet clear what the best method is for predicting response to CRT. Several imaging modalities, such as magnetic resonance imaging (MRI), echocardiography, planar scintigraphy and single photon emission computed tomography (SPECT), have attempted to establish methods that provide a substantial improvement in

patient selection for CRT. SPECT RNA imaging is one possibility and is the focus of this thesis. This chapter provides background on the physics of SPECT and also discusses what the other imaging techniques have accomplished upon tackling the problem of CRT non-response. 


\subsection{Physics of SPECT}

\subsubsection{Gamma-ray emission}

Nuclear medicine consists of three imaging modalities: planar scintigraphy, single photon emission computed tomography and positron emission tomography (PET). Nuclear medicine relies on the emission of radiation from inside a body to be detected and analyzed by external detectors.

As its name implies, SPECT utilizes the emission of photons to produce images. Photonemitting radionuclides possess an unstable nucleus that will release its excess energy in the form of a gamma-ray. Often, the release of a gamma-ray is preceded by a beta emission which leaves the nucleus in an excited state. If the half-life following the beta decay is relatively long, the atom is called an isomer and is said to be in a metastable state. A typical decay scheme for a beta decay and subsequent isomeric transition is depicted below:

$$
{ }_{Z}^{A} X \rightarrow{ }_{Z+1}^{A} Y^{*}+\beta^{-}+\bar{v} \rightarrow{ }_{Z+1}^{A} Y+\gamma
$$

where $\mathrm{X}$ the radioactive parent decays into $\mathrm{Y}^{*}$ (the radioactive daughter in an excited state), an electron $(\beta-)$ and an anti-neutrino $(\bar{v})$. The radioactive daughter then releases a gamma-ray $(\gamma)$ to fall to a ground state, $Y$. 
As it travels, a gamma-ray photon is susceptible to interaction with its surrounding medium via three processes: pair production, photoelectric effect and Compton scattering.

Pair production occurs in the vicinity of an atom when the photon is converted into an electron-positron pair. For this process to occur the photon energy must be greater than $1.022 \mathrm{MeV}$ and so this interaction occurs significantly only at high gamma-ray energies. In SPECT imaging, the radionuclides used typically emit photons with energy on the order of hundreds of $\mathrm{keV}$ or less and thus pair production is not a factor.

The photoelectric effect occurs when the photon's energy is completely transferred to a nearby atom, thereby releasing an electron from the atom. The electron escapes with energy of the incident photon minus the binding energy required to liberate the electron. Photoelectrons will not contribute to the detected signal as they will likely not reach the detector because the range of an electron in tissues is only a few millimeters.

Compton scattering occurs when a photon scatters upon collision with an electron. As opposed to the photoelectric effect, only a part of the photon's energy is transferred to the electron and as such both the photon and electron are scattered in different directions in order to satisfy conservation of momentum and energy laws. Several instances of Compton scattering may occur for a single photon. Many Compton scattered photons will deflect away from the detector but some will still be detected with an incorrect direction of incidence due to the scatter event. However, as will be discussed in section 
2.1.3.3, the number of Compton scatter events detected can be reduced based on the lower energy signal that they will create.

\subsubsection{Radiotracers}

In SPECT imaging, a gamma-ray emitting radionuclide is bound, or tagged, to a desired pharmaceutical compound, creating a radiotracer which is distributed inside the body, following the physiological function of the compound. The radiotracer is chosen with the purpose that the uptake of the compound accumulates in a desired localized region, such as an organ, and the properties of the compound are undisturbed by the presence of the radionuclide. The radiotracer emits gamma-rays but only a small fraction of them will reach the detector due to the isotropic emission, attenuation and the inverse square law.

The choice of radionuclides for SPECT depends on several factors, such as, half-life, emission energy, availability and cost as well as its ability to attach to a specific compound. There are several hundred radionuclides which can be used in nuclear medicine, yet only a fraction of them are suitable for SPECT imaging (51). Table 2.1 below summarizes common SPECT radionuclides as well as their uses and properties. 
Table 2.1: Properties of common radionuclides used in SPECT imaging along with typical clinical usage. Data obtained from Prince and Links (51).

\begin{tabular}{llll}
\hline Radionuclide & $\begin{array}{l}\text { Half-life } \\
\text { (hours) }\end{array}$ & $\begin{array}{l}\text { Photon energy } \\
(\mathbf{k e V})\end{array}$ & Imaging usage \\
\hline Technetium-99m & 6.01 & 140 & Heart, bone, kidney, lung \\
Gallium-67 & 79.2 & $92,184,296$ & Tumor, infection, inflammation \\
Indium-111 & 67.2 & 173,247 & Infection, inflammation \\
Iodine-123 & 13.3 & 159 & Thyroid, kidney \\
Iodine-131 & 193.0 & 364 & Thyroid \\
Xenon-133 & 127.2 & 81 & Lungs \\
Thallium-201 & 73 & 135,167 & Myocardium \\
\hline
\end{tabular}

An ideal SPECT radiotracer must possess a relatively long half-life (minutes to hours) so that there is enough activity present to produce a measurable signal following production, injection, and duration of the acquisition. However, the radiotracer half-life must be short enough so that a) sufficient counts are acquired in a reasonable time frame and b) no significant amount of radioactivity remains in the patient for long periods following the medical procedure.

The radiotracer emissions must ideally be a high yield monoenergetic gamma-ray so that there is no mixture of particles detected which would reduce image quality. The energy of the gamma-rays must be high enough to escape attenuation of the body, limiting patient dose and maximizing the signal that reaches the detector. However, the energy 
must be low enough to ensure that the gamma-ray a) interacts with the detector and b) does not penetrate the collimator septa (see section 2.1.3.1), blurring the image (52).

By far the most common radionuclide used in nuclear medicine is Technetium-99m, or Tc-99m (52). The decay scheme of Tc-99m is given below.

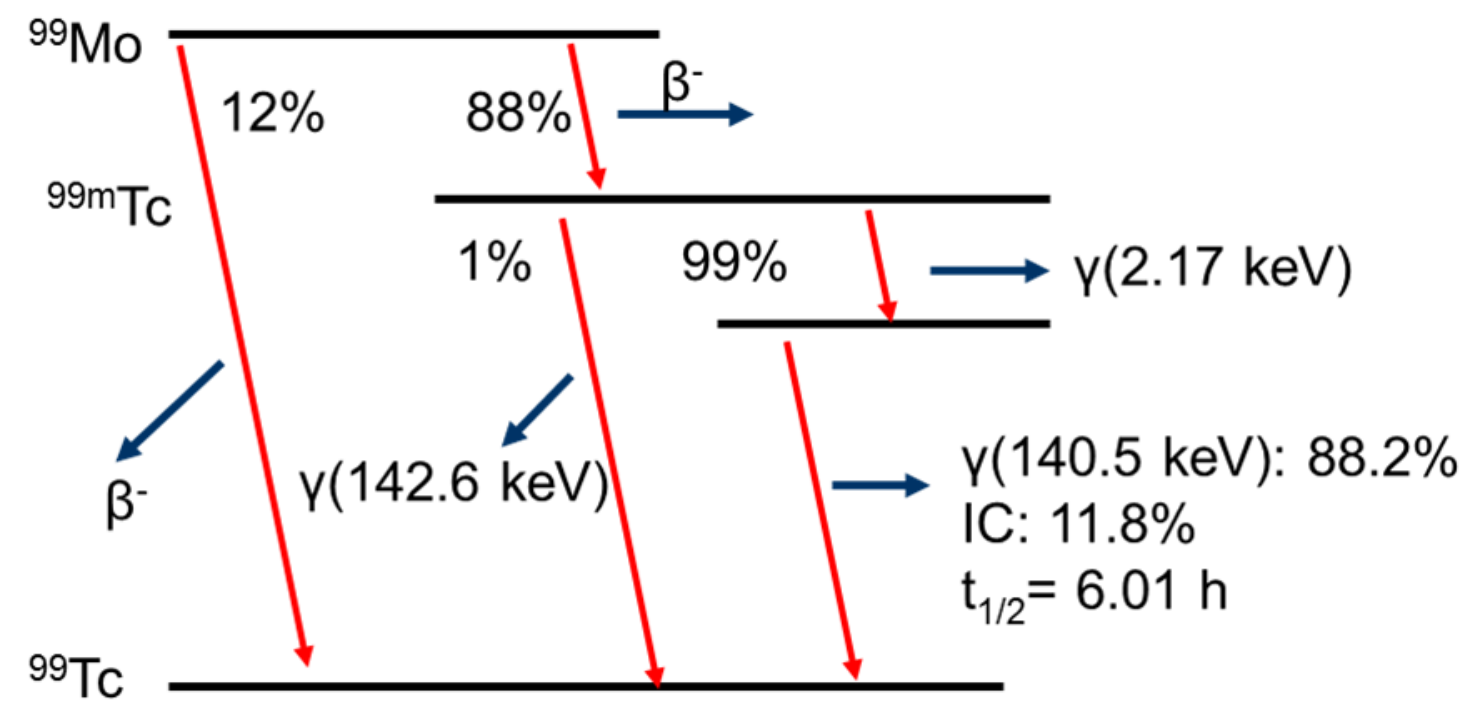

Figure 2.1: Decay scheme of Technetium-99m. The parent isotope, Molybdenum-99, decays with a yield of $88 \%$ via $\beta$ - emission into Tc-99m, which then decays via gamm-ray $(\gamma)$ emission $(99 \%)$ to another excited state. Finally, Tc-99m decays to Tc-99 either by gamma-ray emission (140.5 keV, yield of $88.2 \%$, or by internal conversion (IC) (11.8\%). Decay scheme adapted from National Nuclear Data Centre (53)

As discussed above, Tc-99m possesses an ideal half-life and gamma-ray energy for use in nuclear medicine. Molybdenum-99 (Mo-99), the radioactive parent of Tc-99m, is a fission product of U-235, possesses a half-life of 66 hours and decays via beta minus emission with an $88 \%$ yield to Tc-99m (53) (Figure 2.1). There exist several compounds 
to which Tc-99m can be attached, depending on the desired physiological function to be investigated. In cardiac imaging, Tc-99m can be bound to red blood cells (RBC) to perform SPECT radionuclide angiography (RNA) (or SPECT gated blood pool imaging); it is then possible to follow the circulation and accumulation of blood within a body, such as in blood vessels and in the chambers of the heart. Other Tc-99m radiotracers can be used in cardiac imaging for myocardial perfusion, thereby tracking the flow of blood in the heart muscle.

In this thesis, images are created following the injection of Tc-99m labeled RBCs. There are three common techniques currently used to label RBCs with Tc-99m; in-vitro, in-vivo and modified in-vivo. In each technique, the RBCs are mixed with $\mathrm{Sn}^{2+}$ ions prior to the addition of ${ }^{99 \mathrm{~m}} \mathrm{TcO}_{4}{ }^{-}$which enables the stannous compound to reduce the Tc-99m from +7 to a lower oxidation state that is more reactive which will readily bind to RBCs with high efficiency. In the in-vitro method (54), a blood sample is first extracted from the patient and put in a centrifuge to separate the RBCs from the other blood constituents. A few minutes later, the $\mathrm{Sn}^{2+}$ solution is added to the $\mathrm{RBCs}$, followed later by the introduction of the ${ }^{99 \mathrm{~m}} \mathrm{TcO}_{4}{ }^{-}$solution. The in-vitro technique provides very high efficiency RBC labeling ( $>97 \%$ ), but the procedure is time consuming and the required equipment is not readily available in all clinics (54). In the in-vivo injection technique (54), a stannous solution is first injected in the patient intravenously. After the stannous solution has distributed in the blood, the ${ }^{99 \mathrm{~m}} \mathrm{TcO}_{4}{ }^{-}$solution is intravenously injected and is allowed time to distribute, reduce and bind to RBCs. The labeling efficiency of the invivo method is lower than the in-vitro method (approximately $80-90 \%$ ). Though the 
technique is simpler to implement, there is a significant amount of undesired radiotracer uptake in extravascular bodies. The modified in-vivo technique, initially suggested by Callahan (55), first introduces a stannous compound by intravenous injection. Following a 20-30 minute period, a small blood sample is extracted from the patient where it is mixed with the ${ }^{99 \mathrm{~m}} \mathrm{TcO}_{4}{ }^{-}$solution for labeling. The radiotracer-labeled blood sample is then re-injected into the body, where the radiotracer is given another 20-30 minutes to distribute and metabolize in the body. The advantage of the modified in-vivo is that the Tc-99m is given time to bind to RBCs in isolation before competing body compartments, such as the gastric mucosa and thyroid, take up the radiotracer. The modified in-vivo technique improves on the labeling efficiency of the in-vivo technique while remaining relatively simple to perform. It is especially useful for reducing background activity and ensuring maximal activity in the heart.

\subsubsection{Gamma Camera}

Once the radiotracer is injected and sufficient time is allowed for uptake, the patient will be placed under an Anger gamma camera, as depicted in Figure 2.2. The photons are emitted isotropically and may undergo scatter or attenuation in the body before reaching the Anger camera. Therefore, only a small fraction reaches the detector. The Anger camera consists of a lead collimator, a large scintillation crystal, an array of photomultiplier tubes (PMT) and circuitry to analyze the position, pulse height and timing of each detected event. 


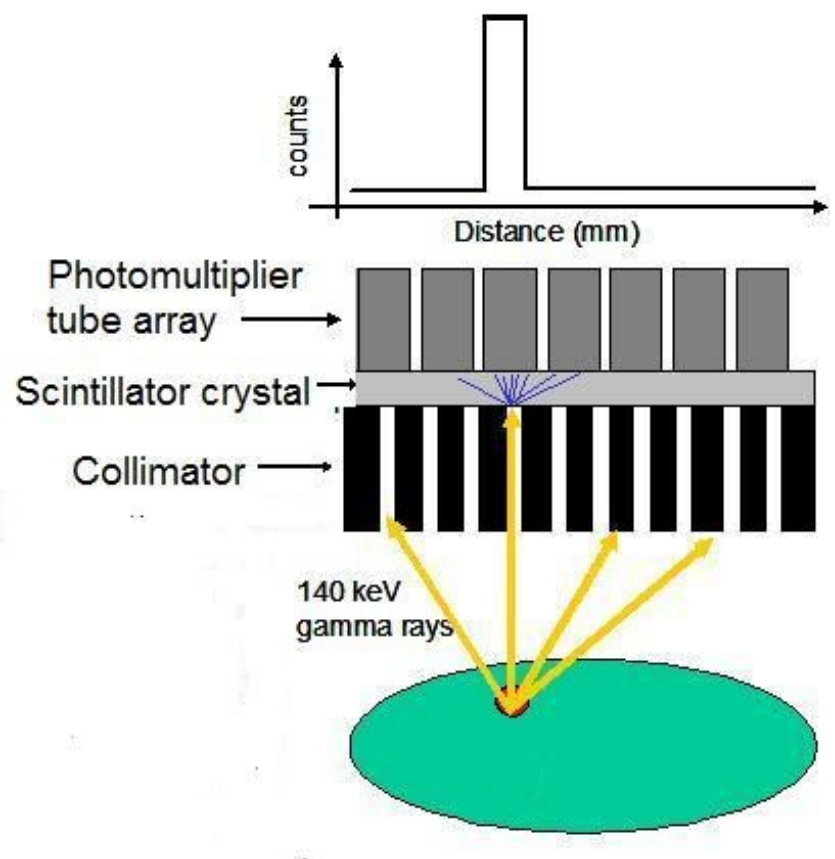

Figure 2.2: Schematic diagram of an Anger camera (not to scale). The collimator system helps with directionality as it absorbs all gamma-rays that are not perpendicular to the scintillation crystal. The scintillator will absorb incident-gamma-rays and emit scintillation photons that will be interact with the photomultiplier tube array and produce a measurable signal used to determine the pulse height and location.

\subsubsection{Collimator}

A single lead septa separating each hole is approximately $30-40 \mathrm{~mm}$ in length, $0.20 \mathrm{~mm}$ wide while the holes are 1-3 mm in width (51). Collectively, they span across the entire underlying scintillation crystal (Figure 2.2) and work to allow only certain incident angle photons (those not absorbed by the collimators) to reach the underlying scintillation crystal. Usually, parallel hole collimators are used for which the holes are perpendicular to the crystal. Converging, diverging and pinhole collimators can also be used. 
Collimators limit the direction of travel of detected photons which ensures that they can properly be traced back to the field of view.

\subsubsection{Scintillation crystal}

Incident photons that successfully travel through the collimator holes are incident upon a scintillation crystal. The most commonly used type of scintillation crystal for photon detection in nuclear medicine is a thallium-doped sodium iodide crystal $(\mathrm{NaI}(\mathrm{Tl}))$. Dimensions of the crystal vary, but a thickness ranging from 5-25 mm, with the most common thickness being $9 \mathrm{~mm}$, and a rectangular surface of $40 \times 50 \mathrm{~cm}$ are typical (51). Thicker crystals will increase interaction probabilities, and thus efficiency, but provide inferior spatial resolution.

A $\mathrm{NaI}(\mathrm{Tl})$ crystal is an inorganic scintillator that possesses excellent light output, a relatively high density of $3.7 \mathrm{~g} / \mathrm{cm}^{3}$ and a light decay time constant of $230 \mathrm{~ns}(52,56,57)$. The relatively high density of $\mathrm{NaI}(\mathrm{Tl})$ ensures a high efficiency at stopping the incident photons while the time constant represents the time required from absorption to fluorescence which plays into the dead time of the system. In an inorganic crystal, the electrons are located in bands separated by energy gaps. The uppermost bands are called the valence band, which is generally full of weakly bound electrons, and the conduction band, which is empty. Doping a NaI crystal with Thallium introduces impurities in the crystal, which create activator energy states (traps) in the energy gap between the valence and conduction bands in the crystal lattice. The incident gamma-ray photons interact with the scintillator via Compton scattering and the photoelectric effect, causing 
electrons in the valence band of the crystal lattice to be raised to excited states, landing either into traps or into the conduction band. The excited electrons then return to valence band emitting a photon of wavelength of near-visible to visible light. Those returning to the valence band from the conduction band are energetic enough to re-excite the crystal and thus do not reach the PMTs. The electrons returning to the valence band from a trap are said to be transparent to the crystal as they each release a photon whose energy is below the amount needed to re-excite the crystal. These photons will then reach the PMTs for conversion and amplification into an electrical signal. A single incident gamma-ray photon can produce several thousand scintillation photons.

\subsubsection{Photomultiplier tubes (PMT)}

An array of PMTs is positioned behind the scintillator to detect the scintillation photons. Each PMT tube is comprised of a photocathode, a series of dynodes, each set at a progressively higher voltage and an anode. Most photons incident on a PMT interact by the photoelectric effect with the photocathode. The liberated electrons are then accelerated to the first dynode. Upon colliding with the dynode, the electron's kinetic energy is transferred liberating 3-6 additional electrons, amplifying the signal. These electrons are accelerated to the next dynode and the signal is further amplified at each subsequent dynode as this process is repeated until it reaches the anode where the final pulse signal with a total amplification of $10^{6}-10^{8}$ is recorded $(51,56)$. An array of PMTs is necessary for two reasons. First, to detect photons across the full area of the scintillator because the dimensions of a single PMT is significantly smaller than the scintillation crystal. Second, with an array of PMTs, the several thousand scintillation photons 
produced by a single gamma-ray absorption are distributed over many PMTs. Thus the PMTs can be used for event positioning logic as well as total pulse height measures. The signals measured in each PMT can be summed in order to obtain a total signal, called a zpulse, which is proportional to the energy of the incident gamma-ray (equation 2.2). Incident gamma-rays that were scattered in the body can be rejected if it is determined that their z-pulse (i.e. energy of the incident photon) is outside a pre-set energy window. Finally, the centre of mass of the PMT signals is calculated and used to determine the position of the incident gamma-ray on the detector. Each accepted event produces a relative $\mathrm{X}$ and $\mathrm{Y}$ position (equations 2.3 and 2.4), and a count of one is added to a 2dimensional histogram at that particular location. The gamma-ray is then determined to have originated along the volume defined by the pixel size extending in a direction parallel to the collimators.

$$
\begin{gathered}
Z=\sum_{k=1}^{K} a_{k}, \\
X=\frac{1}{Z} \sum_{k=1}^{K} x_{k} a_{k}, \\
Y=\frac{1}{Z} \sum_{k=1}^{K} y_{k} a_{k},
\end{gathered}
$$

where $\mathrm{Z}$ is total pulse height, $\mathrm{a}_{\mathrm{k}}$ is the pulse height measured in the $\mathrm{k}^{\text {th }} \mathrm{PMT}$ and $\mathrm{x}_{\mathrm{k}}$ and $\mathrm{y}_{\mathrm{k}}$ are the positions of the $\mathrm{k}^{\text {th }}$ PMT. 
A single static $2 \mathrm{D}$ image is created by summing the counts over the course of the whole scan. However, if the activity distribution or concentration is expected to vary during the course of the scan, a dynamic scan may be required.

Using advanced circuitry, the timing of an event, $t$, can be recorded along with its $\mathrm{X}, \mathrm{Y}$ and $\mathrm{Z}$ values. This is especially useful in cardiac imaging. Using the time interval between subsequent R-waves of an ECG to denote a single heartbeat, it is possible to divide one cardiac cycle into several gates, or time frames (Figure 2.3). During the first time interval following an R-wave (gate 1), all events are recorded in a matrix. Once the time interval for a single bin has elapsed, all events recorded are then assigned to a second matrix representing gate 2 . This process is repeated for the number of frames that the cardiac cycle is divided into. Once the R-wave re-occurs on the ECG, data are once again assigned to the first matrix, and the process repeats itself. This is known as a multiple gated-acquisition (MUGA) and enables the production of images from each individual time-interval or gate. From this time series of images it is also possible to produce a cine movie of the beating heart. MUGA imaging is advantageous over static frame imaging because the data will not be averaged over the cardiac cycle and information about the changes in heart size or activity distribution during contraction and relaxation can be obtained. 


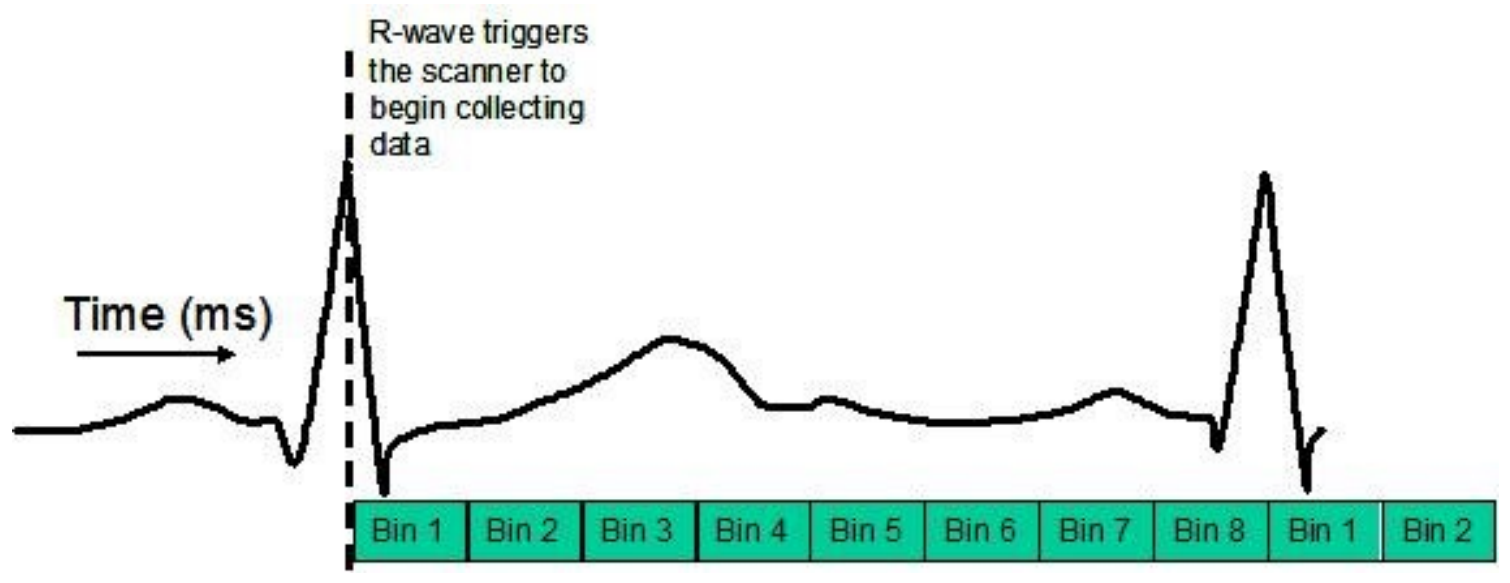

Figure 2.3: Sample R-R wave electrocardiogram divided into 8 time bins, or gates.

\subsubsection{SPECT Acquisitions}

Tomography refers to the creation of a 3D image and, in the case of SPECT, to a 3D image of the radiotracer distribution in the patient. Up until now, all the physics described have been limited to a single static Anger camera obtaining a single planar twodimensional image. In order to obtain a true SPECT acquisition, it is necessary to acquire several projection images, which will then undergo reconstruction into several transverse slices, producing a 3D image. In SPECT systems, the hardware includes a gantry designed to rotate the Anger camera, allowing acquisition of projections each at different angles. Several SPECT systems also incorporate a second Anger camera, often placed at a $90^{\circ}$ orientation with respect to the first camera, to cut acquisition times in half by acquiring two projections simultaneously. Each projection corresponds to a standard planar image and is acquired for a fixed period of time to obtain sufficient counts. The gantry is then rotated and a new projection is acquired. 
After all projections have been acquired (complete rotation of Anger cameras across $180^{\circ}$ ), the data are reconstructed into a collection of transverse slices image using filtered backprojection or iterative reconstruction. The reconstructed image becomes a threedimensional representation of the activity distribution within the field of view. Many different reconstruction algorithms have been developed and are available on commercial SPECT camera systems. The details of image reconstruction are not the focus of this thesis, but the interested reader will find good reviews in the following references $(51$, $58-60)$.

\subsubsection{Cardiac Orientation}

In cardiac imaging, the typical body reference axes (transverse, coronal, sagittal) are reoriented to better represent the orientation of the heart inside the body. The volume is re-oriented so that the LV long axis is defined by the longest dimension from LV apex to central mitral valve plane $(61,62)$. This orientation helps with proper visualization of the cardiac chambers and coronary blood flow (61). Slices obtained along the short axis perpendicular to the LV long axis are called short axis (SA) slices (63). Based on the short axis reference view, slices cut in the vertical plane are defined as vertical long axis (VLA) while slices in the horizontal plane, perpendicular to the septal wall between the ventricles are along the horizontal long axis (HLA) (63). A sample SPECT RNA reconstructed image displayed in all 3 reference axes is shown in Figure 2.4. 

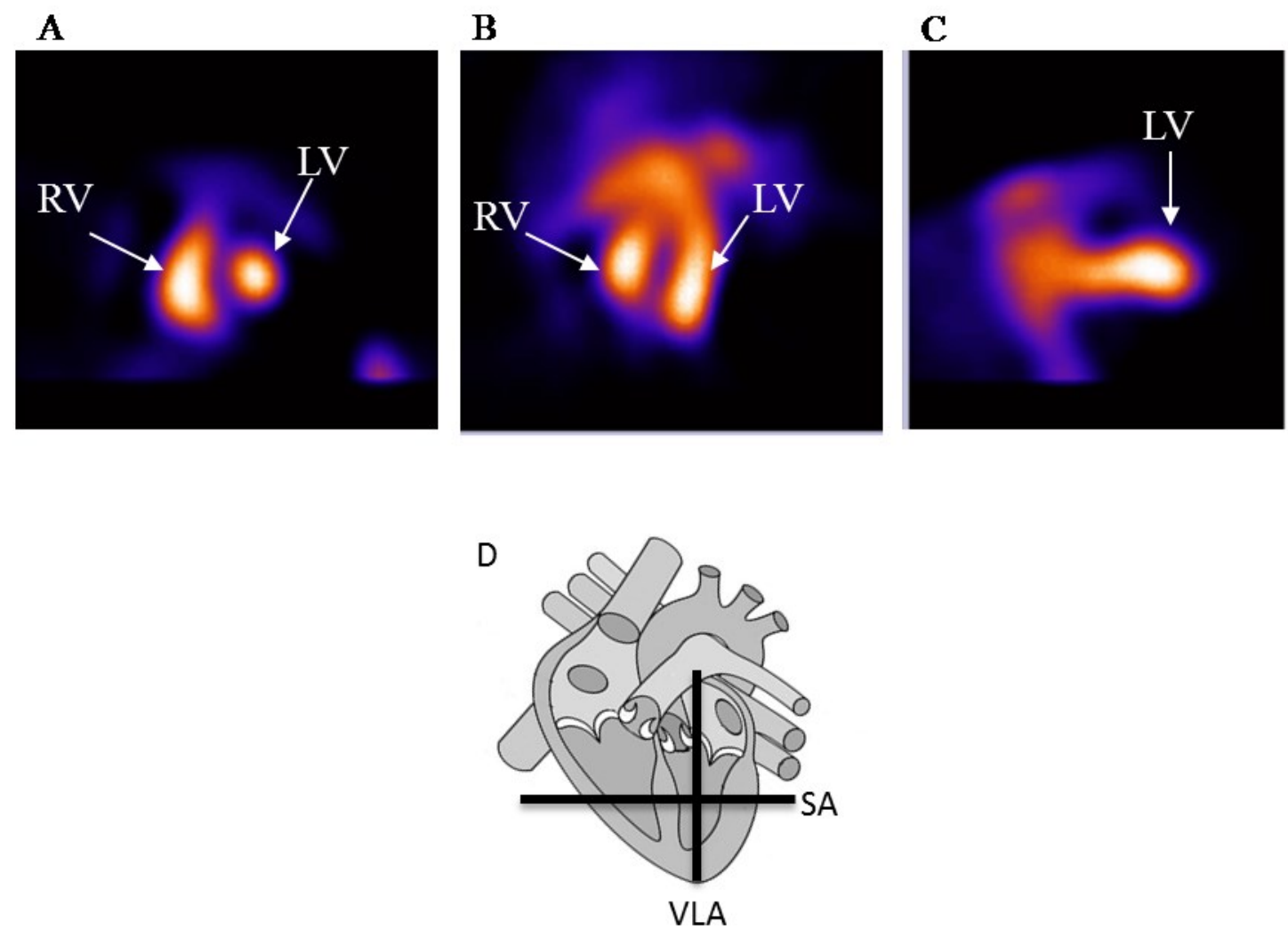

Figure 2.4: SPECT RNA reconstructed image at a) SA view, b) HLA view and c) VLA view at an arbitrary slice. The HLA and VLA images were obtained by a cut through the center of the LV in the SA. d) A schematic view of the HLA orientation along with the planes of the SA and VLA. Image d) modified from image obtained from http://www.sciencekidz.co.nz/ (4)

In comparing the cardiac reference frame to the typical body frame, the SA is analogous to the transverse plane in the body, while the HLA and VLA are analogous to the coronal and sagittal planes respectively.

The LV of the heart is often depicted in polar map format, a two dimensional representation of the LV (Figure 2.5) where the center of the polar map represents the LV apex and the outer rings represent basal slices. Polar maps are often used for segmental analysis of the $\mathrm{LV}$. 


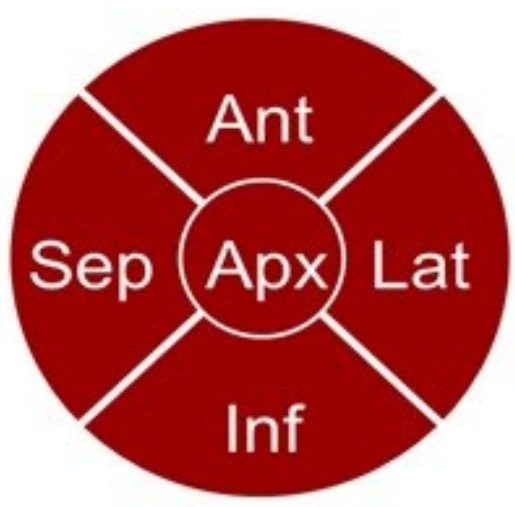

Figure 2.5: Sample 5-segment polar map representing a two dimensional view of the left ventricle, from apex (middle) to the basal slices (outer rings). Ant $=$ Anterior, Lat $=$ Lateral, Inf $=$ Inferior, Sep $=$ Septal and Apx $=$ Apex.

\subsection{RNA MD evaluation}

As discussed in chapter 1 , there is a need to establish quantifiable MD parameters to better understand why certain patients do not respond to CRT, but also, ultimately, to improve the patient selection criteria for CRT.

MD causes the poor pumping efficiency observed in CHF patients and can be expressed by examining the physical contraction of the LV. This can be achieved by analyzing wall motion. The presence of abnormal wall motion will adversely affect the pumping efficiency. Wall motion quantification can be achieved by Fourier analysis (FA) which produces parameters to characterize MD.

RNA images the distribution of blood as a function of time within blood vessels and chambers, such as the LV, facilitating wall motion tracking, thus making it an ideal modality for FA purposes. SPECT RNA is superior to planar RNA as it allows the LV 
wall to be defined from many slices rather than a single planar view, increasing the amount of data points available for FA. The 3D nature of SPECT RNA minimizes the effects of shadowing due to overlapping structures that occurs with planar RNA. Because the acquired data are divided over multiple projections, fewer gates are usually recorded with SPECT RNA compared to planar RNA to obtain sufficient counting statistics in a reasonable time frame. Chen et al. (64) have shown that performing FA on data acquired at 8 gates could still resolve phase differences up to $5.6^{\circ}$.

\subsubsection{Fourier Analysis}

In FA, two possible wall motion analysis techniques are possible, time-distance (TD) and time-activity (TA). Both techniques were evaluated by Lalonde et al. (65), and it was determined that the TA method is superior to the TD method. In the TD method, the LV wall position is directly tracked at each gate, and thus LV segmentation must be performed at each gate. This leads to very noisy estimates of motion because the magnitude of movement during contraction is on the order of the pixel size, and therefore measuring the actual displacement of the LV wall remains a challenge. In the TA method, wall motion is indirectly measured by observing the change in counts in fixed locations as the heart beats. Thus, it is only necessary to perform LV segmentation at a single time-frame, usually at end-diastole. This reduces the chances of incorrect or indistinct edge detection. In the TA method, partial volume averaging causes the counts in a fixed region of interest (ROI) placed at the LV wall to vary proportionally as the LV wall contracts. The TA method is usually preferred since it can better estimate sub-pixel movement, with partial volume averaging. 
In order to establish MD parameters for SPECT evaluation, the traditional approach has been to perform FA, which consists of approximating a time-activity curve (TAC) as a combination of sinusoidal functions. It is described by Links et al. (66) and summarized below. The general equation for a $\mathrm{K}^{\text {th }}$ Fourier harmonic is given in equations 2.5 to 2.7 . A first order $(\mathrm{K}=1)$ Fourier fit on a TAC, fits the data to a single sinusoidal curve, described in equation 2.8, and the fitted parameters can be calculated to obtain both a phase (P) (equation 2.9) and an amplitude parameter (A) (equation 2.10) representative of the fitted TAC. A first order Fourier fit is typically used for FA as TACs are fit reasonably well. A sample TAC and fit are shown in Figure 2.6 below. The first order phase parameter represents the relative timing of the onset of contraction while the first order amplitude is representative of the extent (magnitude) of contraction. To date, the phase component has been extensively investigated for representing the homogeneity of onset of contraction. This is referred to as phase analysis. Conversely, amplitude analysis has been largely ignored.

$$
\begin{gathered}
F_{K}=\sum_{t=1}^{N}\left(R_{K}-i I_{K}\right), \\
R_{K}=\sum_{t=1}^{N} f(t) \cos 2 \pi K t / N, \\
I_{K}=\sum_{t=1}^{N} f(t) \sin 2 \pi K t / N,
\end{gathered}
$$


where $R_{K}$ and $I_{K}$ represent the real and imaginary components of $F_{K}, f(t)$ is the pixel count at gate $\mathrm{t}$, while $\mathrm{N}$ represents the amount of gates and $\mathrm{K}$ is the order of the harmonic.

$$
F(t)=A \cos (2 \pi t / N-P)
$$

$$
A=\left(R_{1}^{2}+I_{1}^{2}\right)^{1 / 2}
$$

$$
P=\tan ^{-1}\left(I_{1} / R_{1}\right)
$$

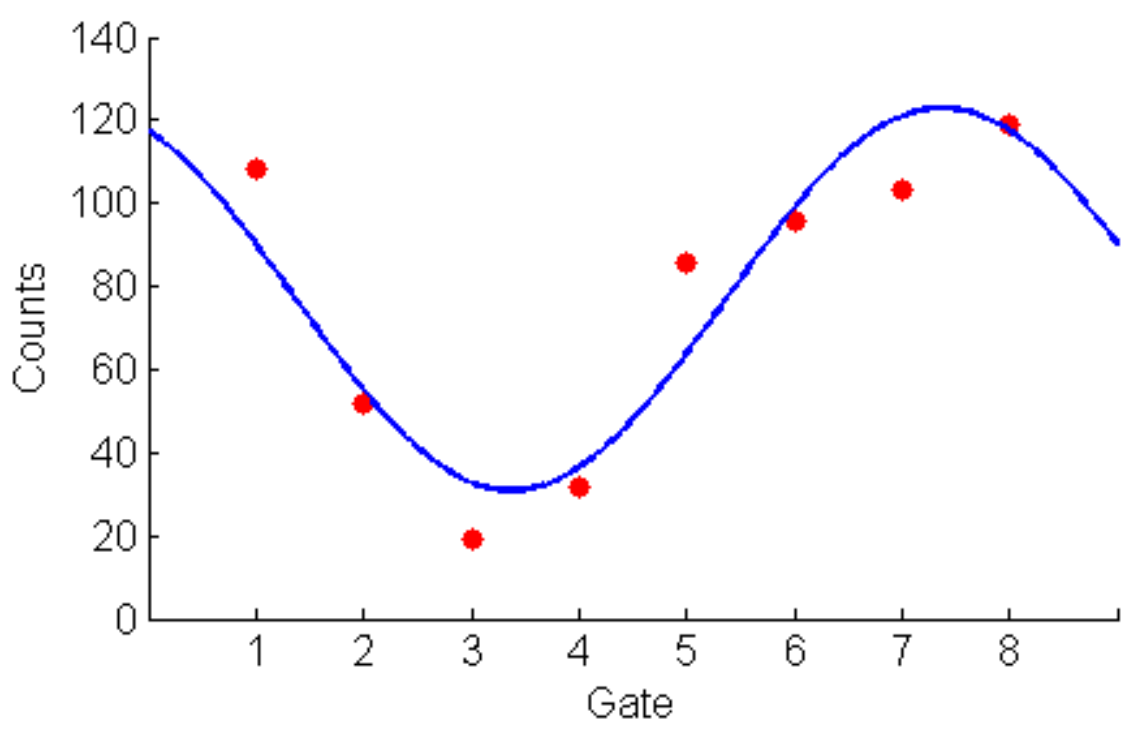

Figure 2.6: Sample first order Fourier fit (blue curve) of actual measured counts (red circles) 


\subsubsection{Phase Histogram}

Several ROIs are typically used to segment the LV and thus several TACs can be

obtained, one for each ROI, making it possible to define statistical parameters for wall motion analysis. A phase histogram, representing the phase distribution, can be plotted for qualitative assessment of dyssynchrony as it depicts the various phase angles (time to onset of contraction) occurring all around the LV wall. The phase histogram of a healthy cardiac patient will produce a single narrow peak (Figure $2.7 \mathrm{a}$ ) as the contraction of the LV is homogeneous, while the phase histogram of a dyssynchrony patient will likely show a broad distribution with one or more peaks (Figure 2.7b). Several statistical parameters have been previously defined for use as MD parameters, such as phase average $\left(\mathrm{P}_{-} \mathrm{AVG}\right)$, phase standard deviation $\left(\mathrm{P}_{-} \mathrm{SD}\right)$, phase-bandwidth $\left(\mathrm{P}_{-} \mathrm{BW}\right)$, phaseentropy $\left(\mathrm{P}_{-} \mathrm{E}\right)$ and synchrony $(\mathrm{S})$. Phase average is not considered a relevant phase analysis parameter as it has been shown that only severe abnormal wall motion produces a perceptible phase change with respect to normal LV wall motion (67). The other FA parameters are used and are described in greater detail in sections 2.2.1.2 to 2.2.1.5 

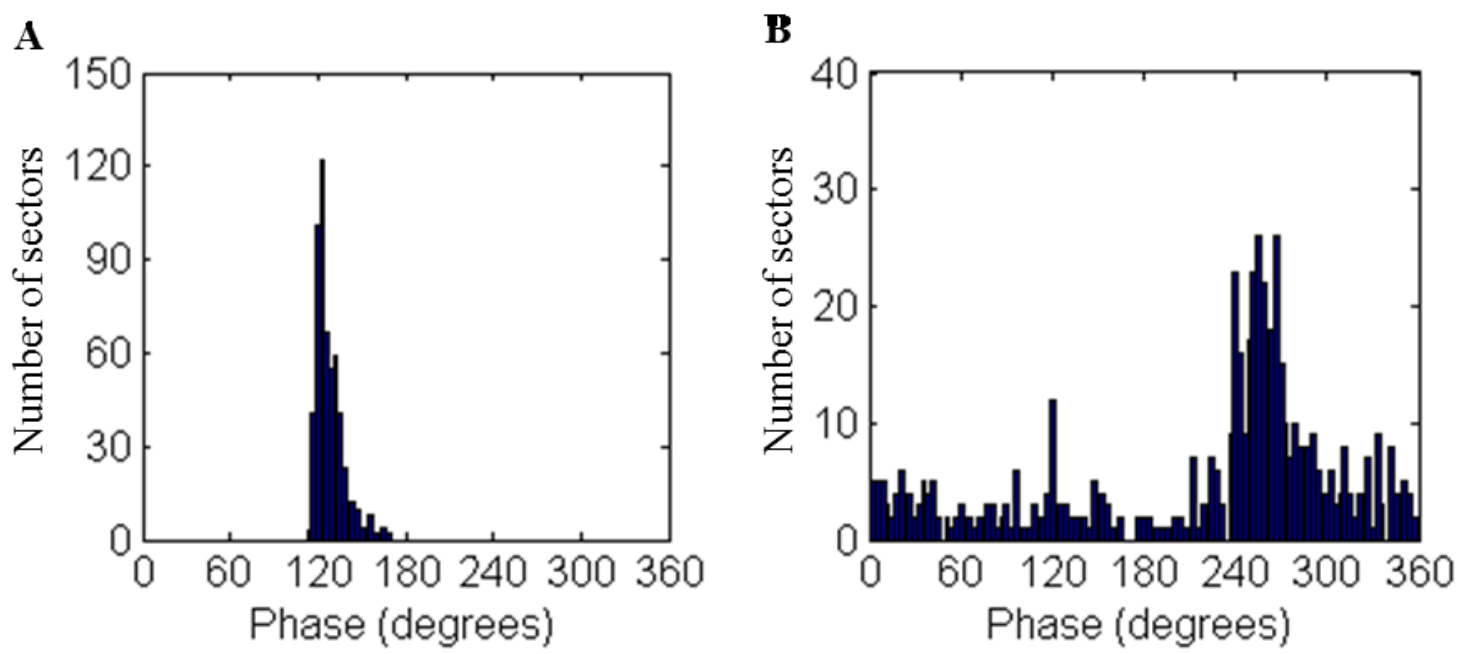

Figure 2.7: Sample phase histogram of a) a normal cardiac patient and b) a dyssynchrony patient.

\subsubsection{Phase Standard Deviation (P_SD)}

P_SD is defined as:

$$
P_{-} S D=\sqrt{\frac{1}{N} \sum_{i=1}^{N}\left(P_{i}-P_{-} A V G\right)^{2}},
$$

where $\mathrm{N}$ is the number of data points, and $\mathrm{P} \_\mathrm{AVG}$ is the average of the phase values, and $P_{i}$ is the phase value of the $i^{\text {th }}$ data point. $P_{-}$SD represents the temporal dispersion of the various phases in the LV and is expressed in degrees from 0 to 360 . P_SD can equivalently be expressed in time units by dividing the phase value by $\left(360^{\circ} *\right.$ heart rate). The spread of the phase values corresponds to the uniformity of contraction timing within the $\mathrm{LV}$ and is a standard measure of wall motion timing. A larger P_SD corresponds to abnormal wall motion and thus greater MD. 


\subsubsection{Phase Bandwidth (P_BW)}

P_BW provides a similar measure of the spread of the data to P_SD. The major difference is that $\mathrm{P} \_\mathrm{BW}$ does not assume the data is normally distributed. It is defined as the difference in the range of phase values which contains $95 \%$ of the data, as defined in equation 2.12 below:

$$
P_{-} B W=P_{97.5}-P_{2.5},
$$

where $P_{i}$ represents the phase value of $i^{\text {th }}$ percentile.

Under most circumstances $\mathrm{P} \_\mathrm{BW}$ is expected to strongly correlate with $\mathrm{P}$ SD. In situations where a near-uniform or a multi-peak distribution is present, $\mathrm{P}$ BW may provide useful information.

\subsubsection{Phase Entropy (P_E)}

P_E, first proposed by O'Connell et al. (68), is based on the Shannon information theorem and is defined in equation 2.13. $\mathrm{P}_{-} \mathrm{E}$ represents the uniformity of movement of the LV and is described as the degree of disorder present in the data. The $\mathrm{P}_{-} \mathrm{E}$ value ranges from 0 to 1 , where 0 is representative of complete order and 1 denotes complete disorder. Complete order is obtained when all phase values are located in a single bin, suggesting that the probability of the next phase value to be placed in that bin is certain.

On the other hand, complete disorder is obtained when the phase values are uniformly 
distributed between all bins, indicating that a subsequent value could fall in any bin with equal probability.

$$
\text { P_E }=\frac{\sum_{i=1}^{M} U_{i} \log _{2} U_{i}}{\log _{2} M}
$$

where $\mathrm{M}$ is the total number of bins and $\mathrm{U}_{\mathrm{i}}$ is the probability of a phase value being placed in bin i. The probability, $\mathrm{U}_{\mathrm{i}}$, for each bin is the fraction of the total data points located in the bin. Wassenaar et al. (69) determined that the ability of P_E to differentiate between normal and dyssynchrony populations was insensitive to bin width. A bin width of $1^{\circ}$ was adopted for this study. A high degree of entropy is indicative of non-uniform wall motion and represents increased MD. P_E may produce similar results to P_SD and P_BW as they all are measures of a histogram shape, more specifically the histogram spread. Of note, $P_{-} E$ is not affected by bin location (i.e. two high probability bins will produce equal P_E whether adjacent or $180^{\circ}$ apart), and therefore $\mathrm{P}_{-} \mathrm{E}$ will indicate a lesser degree of MD for a multi-peak distribution than P_SD.

\subsubsection{Synchrony (S)}

Synchrony is a MD parameter first proposed by O Connell et al. (68) based on both the amplitude and phase parameter obtained from the first order Fourier fit. It represents a normalized vector sum of all data points as described in equation 2.14.

$$
S=\frac{\left|\sum_{i=1}^{N} \vec{v}_{i}\right|}{\sum_{i=1}^{N}\left|A_{i}\right|},
$$


where $A_{i}$ is the amplitude of the $i^{\text {th }}$ data point and $\mathbf{v}_{i}$ represents a vector of magnitude $A_{i}$ and direction given by the phase, $\mathrm{P}_{\mathrm{i}}$. The denominator normalizes the vector sum produced by the numerator to the maximum possible amplitude. Thus, $\mathrm{S}$ ranges from 0 to 1 , where 0 is representative of complete dyssynchrony and 1 denotes complete synchrony. Complete dyssynchrony occurs if the vector sum cancels out (i.e. isotropic distribution or multiple peaks $180^{\circ}$ apart), while complete synchrony occurs if all vectors possess the same phase value.

\subsubsection{Inter-ventricular dyssynchrony}

Inter-ventricular dyssynchrony is usually represented by calculating the average phase difference between the RV and LV. Some studies (70-72), but not all $(73,74)$, have shown that inter-ventricular dyssynchrony is predictive of CRT response. It is possible that the relative difference in timing of contraction between ventricles may provide some insight into the issues of non-response to CRT, but it is believed that intra-ventricular dyssynchrony is the major key factor in MD analysis. In this thesis, the focus is on intraventricular dyssynchrony analysis and measures of inter-ventricular dyssynchrony are not explored.

\subsubsection{SPECT RNA Software Analysis}

In this study, in-house software developed and described by Lalonde et al. (65), was used to perform LV segmentation and subsequent FA. The user helps define the general location of the LV by positioning a box around the LV in all orientations (SA, HLA, 
VLA). The software then proceeds automatically to resample the LV into 18 SA slices, from apex to base, as well as approximate the center of mass (COM) of the LV. From the COM, at each SA slice at end-diastole, the software searches radially outwards, at pre-set angles, until the counts fall below a pre-defined threshold value with respect to the maximum pixel count inside the LV, or until the search encounters a local minimum. In either case, a marker is placed at that location to define the LV edge. In total, the LV wall is marked by 568 points and each of these points is then used to create a rectangular region of interest (ROI) 3 pixels long by 2 pixels wide. Each ROI is centered at the LV wall and is rotated so that the long side is oriented towards the LV COM. The location of each ROI is then fixed with respect to the camera field of view as the heart beats, and the total counts in each frame is recorded and used to plot a TAC. An ROI larger than a pixel enables the time-activity based method to be less sensitive to small variations in LV segmentation. Each of the 568 TACs is then fit to a first Fourier harmonic for FA as described above. Due to the semi-automatic nature of the segmentation algorithm, the inter- and intra-patient reproducibility of FA parameters are excellent, producing Pearson correlation coefficients of 0.96 or greater for both inter- and intra-observer variability (65). The software was also validated by differentiating MD patients from normal cardiac patients with a receiver operating characteristic (ROC) area under the curve (AUC) $\geq$ 0.93 for P_SD, P_E and S (65). A brief overview of ROC analysis is given in section 2.2.3. 


\subsubsection{ROC Analysis}

ROC analysis is a tool for evaluating the performance of a diagnostic test. The ROC curve plots out the sensitivity of a test versus one minus its specificity as a function of the test-value used to distinguish true (positive) from false (negative) test results. For any given cutoff test value, sensitivity is defined as the ratio of positive test results that have correctly identified positive cases over the total number of actual positive cases. Similarly, specificity is defined as the ratio of correctly identified negative test results over the total number of actual negative cases.

The area under an ROC curve (AUC) is often used as a measure of the strength of the test. Ideally, a test will produce perfect sensitivity and specificity and, therefore, will produce an ROC AUC of 1. Conversely, a test with no predictive power will correctly classify data in a binary system (positive or negative) $50 \%$ of the time producing an ROC AUC of 0.5 , and this ROC curve is known as the chance line. The ROC AUC is an important parameter as it has been shown to correspond to the probability that the test correctly classifies a patient (75).

For the interested reader, the following references by Zweig et al. (76) and Fawcett (77) provide more detailed information on ROC analysis.

\subsubsection{Dyssynchrony evaluation}

Both planar and SPECT RNA studies have demonstrated that phase analysis parameters could assess MD by characterizing abnormal cardiac $(67,78-81)$ patterns and be a 
predictor of cardiac events $(74,82)$. Other studies have also shown that phase analysis could differentiate between normal and MD patients with high accuracy $(65,68,69,83)$.

Badhwar et al. (84) studied the correlation between improvement in NYHA class (pre- to post-CRT and change $(\Delta)$ in LVEF, $\Delta \mathrm{P}_{-} \mathrm{SD}, \Delta \mathrm{P}_{-} \mathrm{E}$ and $\Delta \mathrm{S}$ assessed by planar RNA following CRT in $46 \mathrm{CHF}$ patients. The study showed that $\Delta \mathrm{P} \_\mathrm{E}$ and $\Delta \mathrm{S}$ correlated best with clinical improvement (84). In the same study population, Botvinick et al. (73) determined that a combination of baseline planar RNA $\mathrm{P} \_\mathrm{E} \geq 0.69$ and $\mathrm{S} \leq 0.87$ predicted NYHA class improvement in $86 \%$ of patients. Baseline LVEF and P_SD did not predict CRT outcome (73).

Badhwar et al. (85) investigated whether SPECT RNA and planar RNA produced equivalent phase analysis parameters. They determined that SPECT RNA values produced statistically significantly greater values in $\mathrm{P} \_\mathrm{E}(\mathrm{p}<0.01), \mathrm{P} \_\mathrm{SD}(\mathrm{p} \leq 0.05)$ and a smaller value of $S(p<0.01)$ compared to planar RNA (85). All these results indicate that planar RNA may underestimate the severity of MD by analyzing data from a single projection.

\subsection{Other Imaging Modalities}

Every imaging modality has investigated the problem of CRT non-response in an attempt to better understand the underlying mechanisms. The majority of the imaging modalities have mainly focused on establishing MD parameters, but some modalities, including SPECT MPI, PET and MRI have also investigated the effect that the presence of LV scar 
may have on CRT response. For each relevant imaging modality, a summary of the studies conducted concerning dyssynchrony and scar assessment is given below.

\subsubsection{SPECT MPI}

The underlying physics in SPECT perfusion imaging are identical to RNA imaging. The major difference occurs from the radiotracers used, which are taken up in the blood vessels (including the major coronary arteries that supply blood to the heart muscle itself. Therefore, in MPI imaging, the distribution of radiotracer as a function of time permits measurement of wall motion through the change in myocardial wall thickness. FA analysis can then be performed on the data in an identical manner to RNA imaging.

It is possible to quantify scar with MPI techniques as reduced uptake of the radiotracer (i.e. reduced or absent perfusion) in localized regions of the LV is indicative of fibrotic scar tissue.

\subsubsection{Dyssynchrony evaluation}

The majority of SPECT MPI studies use the Emory Cardiac Toolbox (86) for wall motion analysis. The software performs FA using the TA method and can output basic FA parameters such as those described previously. Trimble et al. (87) validated the technique, demonstrating that FA parameters measure MD by showing that various phase analysis parameters can differentiate between various dyssynchrony patient groups. The study found that all phase analysis parameters investigated were able to differentiate between normal cardiac patients, LBBB, RBBB, LV dysfunction (LVEF < 40\%) and RV 
paced rhythms (87). Van Kriekinge et al. (88) also showed that both global (P_E, P_SD, P_BW) and regional phase analysis (phase difference between septal and lateral wall) parameters could successfully differentiate between normal cardiac patients and those with LBBB.

Henneman et al. (89) investigated the ability of several phase analysis parameters to predict response to CRT in a group of 42 severe CHF patients. In this population, $\mathrm{P}_{-} \mathrm{SD}$ and P_BW produced good results and were significant predictors of response to CRT. P_SD produced an ROC AUC of 0.78 and an optimal sensitivity and specificity of $74 \%$ at a chosen cutoff value of $43^{\circ}$, while $\mathrm{P}_{-} \mathrm{BW}$ produced an ROC AUC of 0.78 and a $74 \%$ sensitivity and specificity at a $135^{\circ}$ cutoff (89). It is worth noting however, that patients were classified as responders using a subjective end point $(\geq 1$ improvement in NYHA class), which may not be a true measure of response, as was discussed in section 1.3. Boogers et al. (90) produced similar results using a different software package, and indicated that both $\mathrm{P}_{-} \mathrm{SD}$ and $\mathrm{P} \_\mathrm{BW}$ were able to predict CRT response $(\geq 1$ improvement in NYHA class) in $40 \mathrm{CHF}$ patients. The study claimed a sensitivity/specificity combination of $83 \% / 81 \%$ for P_SD and $\mathrm{P}_{-} \mathrm{BW}$ at a cutoff of $19.6^{\circ}$ and $72.5^{\circ}$ (90). Interestingly, the cutoff values varied considerably between the studies suggesting that there may be significant variability between software packages.

\subsubsection{Scar assessment}

The impact that the extent of LV scar has on CRT response has been investigated in a population of 190 ischemic cardiomyopathy CHF patients by Adelstein et al. (91). 
Results indicated that a greater extent of LV scar significantly reduced the chance of event free survival (death, heart transplant, or necessary circulatory support) following CRT (91). It was also shown that ischemic patients with low extent of LV scarring had outcomes similar to non-ischemic patients (91). Ypenburg et al. (92) determined that a direct relation exists between response to CRT ( $\geq 1$ improvement in NYHA class) and the extent of scar tissue. In a population of 51 ischemic patients, responders had significantly lower scar scores compared to non-responders ( $10 \%$ vs. $25 \%$, $<<0.001)$.

SPECT MPI measures wall motion through the change in the myocardial wall thickness as the heart beats. In regions of low perfusion, it is possible that the reconstructed heart wall appears faint or is missing which can make it difficult to segment the LV. Thus SPECT MPI may not be ideal for applications where proper LV segmentation is essential, such as FA. Nevertheless, recently, Cheung et al. (93) addressed this issue in a simulation study and determined that FA could accurately measure phases in regions where perfusion uptake was as low as $10 \%$ of uptake in normal regions.

\subsubsection{Echocardiography}

Echocardiography is an inexpensive and widely available imaging modality that presents no ionizing radiation risks to the patients or staff. It is capable of imaging cardiac anatomy as well as functionality with excellent temporal resolution. The basis of echocardiography consists of transmitting a sound wave into the body in the $\mathrm{MHz}$ range using a transducer. In echocardiography, the waves target the cardiac system and will reflect and scatter off of cardiac tissue surfaces. Echo strength and time from the 
emission to recapture of the sound wave represent some of the information obtained. Echocardiography is a versatile imaging modality and several different acquisition techniques are possible, such as A-mode, M-mode, B-mode, tissue Doppler imaging (TDI) and speckle tracking.

\subsubsection{Dyssynchrony evaluation}

In A-mode, or amplitude mode, the transducer sends out repetitive pulses and plots the signal amplitudes of the reflected waves as a function of time (proportional to depth), as they are detected (51). M-mode, or motion mode, consists of stacking each A-mode signal side-by side horizontally, while the vertical axis represents depth (51). Therefore, each captured echo provides information on the reflection depth while the signal strength is characterized by the brightness at that particular depth. A M-mode study by Pitzalis et al. (94) investigated the time interval between the displacement of the septal and posterior wall during contraction as a marker of dyssynchrony and obtained a sensitivity/specificity

combination of $100 \% / 63 \%$ in a small single study for predicting outcome to CRT. A recent study by Petrovic et al. (95) investigated over 40 echocardiographic and identified two M-mode parameters, left ventricular stroke index and left ventricular fractional shortening, as the best parameters for CRT response prediction (ROC AUC $\approx 0.70$, $\mathrm{p} \leq 0.05)$

TDI enables the analysis of blood flow by using the Doppler Effect to determine the velocity at which low-frequency, high-amplitude, tissue is moving $(96,97)$. It is also possible to obtain total displacement by integrating velocity over time (97). TDI enables 
the measurement of important clinical variables which can be used for MD analysis such as regional peak-systolic velocity and relative timing of contraction (12). Unlike TDI which is limited to longitudinal motion analysis, speckle tracking can also measure cardiac motion in radial and circumferential directions by following speckles over time that appear due to the interaction of the sound waves and myocardial fibers (98). Several small single centre echocardiographic studies have investigated the ability to predict response to CRT using TDI derived parameters (99-106) or speckle tracking parameters with good results (106-109). Of note, Bax et al. identified the time delay to reach peach systolic velocity between the septal and lateral wall as a significant predictor of CRT response ( $\triangle \mathrm{LVESV}>15 \%)$ (99), producing $92 \%$ sensitivity and specificity at a cutoff of $65 \mathrm{~ms}(100)$. Yu et al. (106) reported that the SD of time to peak myocardial contraction in a 12 segment-model represented the best MD parameter for predicting reverse LV remodeling ( $\triangle \mathrm{LVESV}>15 \%$ ) following $\mathrm{CRT}$. Following the positive results of many smaller single centre studies, the PROSPECT trial set out to investigate the ability of a dozen pre-selected echocardiographic parameters, based on published literature, to predict response to CRT in a large multicentre study (110). Results of the PROSPECT study were not favorable as no single parameter produced ROC AUC results above 0.62 for predicting CRT outcome (34). The study also indicated that the majority of the parameters produced moderate to high inter- and intra-observer variability leading to the conclusion that no single echocardiographic parameter may be recommended for use for CRT patient selection (34). 


\subsubsection{Magnetic resonance imaging (MRI)}

MRI consists of placing a patient in a strong magnetic field in order to align the magnetic spins of all tissues in the body along the external field. Localized temporary magnetic fields pulses produced by RF coils then proceed to alter the magnetization vector of the affected tissues, causing it to precess around the main magnetic field as it returns to its equilibrium position. Following this disturbance, the properties of various spin systems such as transverse and longitudinal relaxation (i.e. dampening of the precession) times, are measured as they are characteristic of various tissues. Measurement of these properties enables high resolution and high contrast anatomical imaging. MRI is noninvasive, and is considered a safe procedure with no side-effects.

\subsubsection{Dyssynchrony evaluation}

MRI is capable of three-dimensional cardiac motion analysis and as such can measure LV dyssynchrony using tagging techniques (111) to define longitudinal, radial and circumferential dyssynchrony or by velocity encoded techniques which allows for direct wall motion measurements (112). Few MRI studies have investigated solely dyssynchrony parameters producing comparable results to other modalities using echocardiography defined MD parameters $(113,114)$.

\subsubsection{Scar Assessment}

Several MRI studies have investigated the relation between non-response to CRT and the presence of scar, as assessed by delayed enhancement (DE) MRI (115-119). In DE MRI, 
a gadolinium contrast agent is injected in the patient prior to scanning. The uptake of the contrast agent has been shown to accumulate in areas of irreversible scar tissue, and can therefore be used to quantify LV scar. In one study, Chalil et al. (116) found that the presence of scar in the posterolateral wall in CRT recipients was associated with a poor clinical outcome while in another study, Chalil et al. (117) determined that total LV scar size greater than $33 \%$ was associated with suboptimal responses to CRT. In a small study of 23 patients, White et al. (115) determined that a global LV scar cutoff of $15 \%$ provided a sensitivity/specificity combination of $85 \% / 90 \%$ while a septal scar cutoff of $40 \%$ provided $100 \%$ sensitivity and specificity for predicting CRT outcome. Several other studies indicated that combining a dyssynchrony marker with the presence of scar improves the prediction of CRT outcome (120-123).

MRI has shown promise at predicting CRT outcome but unlike other modalities, is expensive and not readily available in every clinic. Furthermore, MRI imaging is not feasible in all patients, notably in follow-up CRT scans due to the metallic components of the pacemaker device. Consequently, MRI may not be an ideal modality for CRT patient selection.

\subsubsection{Positron emission tomography (PET)}

PET is a nuclear medicine technique similar in operation to SPECT but uses positron emitting radionuclides, instead of gamma-ray emitting radionuclides, to bind to radiotracers. This enables different compounds to be used and thus different functional information to be assessed. Positrons are highly unstable particles which travel a limited 
range before annihilating with an electron to produce two co-linear gamma-rays of 511 $\mathrm{keV}$ each. Using a ring of detectors, the two gamma-rays are detected within a short-time interval of each other. The coincident detection of the two gamma-rays enables a line of response to be traced between the two detectors involved, which crosses the location where the annihilation event occurred. The millions of counts detected in a scan allow the machine to pinpoint the location of the radiotracer with good resolution.

\subsubsection{Dyssynchrony evaluation}

To date, limited work on dyssynchrony has been performed with PET. Pazhenkottil et al. (124) performed phase analysis using the Emory Cardiac Toolbox on both FDG PET scans and SPECT MPI scans of the same patients. A significant correlation existed between FDG PET and SPECT MPI for evaluating P_SD $(\mathrm{r}=0.88, \mathrm{p}<0.001)$ as well as P_BW $(\mathrm{r}=0.88, \mathrm{p}<0.001)$, while there was a 93\% clinical agreement in identifying severe LV dyssynchrony (patients with both $\mathrm{P} \_\mathrm{BW}>135^{\circ}$ and $\mathrm{P} \_\mathrm{SD}>43^{\circ}$ ) between both modalities. Uebleis et al. (125) investigated the ability of global FDG PET phase analysis parameters $\mathrm{P}_{-} \mathrm{SD}, \mathrm{P} \_\mathrm{BW}$ and $\mathrm{P} \_\mathrm{E}$ to predict response to $\mathrm{CRT}$ in a small 14 patient study. Results indicated that only $P_{-} E$ was significantly different $(\mathrm{p} \leq 0.05)$ between responders $(\mathrm{N}=7)$ and non-responders.

\subsubsection{Scar Assessment}

In the same study as described above, Uebleis et al. (125) also determined that LV scar burden was significantly greater in non-responders than responders $(p \leq 0.05)$. Using 
PET/CT hybrid imaging, the study also showed that the pacemaker LV lead was positioned over non-viable myocardium in 4 patients; all non-responders (125).

Birnie et al. (1) investigated the impact of scar on CRT outcome in the PREDICT population. Scar burden was evaluated using a 5 segment model and it was determined that responders had significantly less lateral wall scar than non-responders $(\mathrm{p}<0.01)$. Global and other segmental regions did not possess significantly different scar between the two groups. The statistical significance remained when investigating the subgroups of ischemic and non-ischemic cardiomyopathy patients. Lateral wall scar scores were investigated for their ability to predict response to CRT in subgroups of ischemic (78\% sensitivity and 67\% specificity) and non-ischemic etiology (69\% sensitivity and $78 \%$ specificity) (1). Results from this study were the first to demonstrate that a significant relation exists between lateral wall scar and CRT response in an exclusively nonischemic population.

\subsubsection{Summary of Imaging Modalities}

In summary, all imaging modalities discussed have shown promising results at predicting response to CRT by quantifying either MD or scar or both. However, no single modality can be considered to be the gold standard as each present different drawbacks and none have been able to show definitive and consistent positive results. The presence of LV scar has been shown to cause adverse effects to CRT recipients and can be assessed by MRI, PET and SPECT MPI. Several MD parameters have been developed across all imaging modalities in order to better understand the mechanics behind the non-response 
to CRT. Echocardiography is widely available and is perhaps the most extensively used modality for dyssynchrony evaluation but is highly operator dependent resulting in poor reproducibility. MRI and PET remain mostly underdeveloped in dyssynchrony analysis. FA is a simple technique employed by nuclear imaging modalities which helps to quantify MD by analyzing wall motion. Several studies from planar RNA, SPECT RNA and MPI have shown that FA is a reliable, reproducible technique that is ideally suited to investigate MD. However, further development of novel parameters may be required to improve MD assessment. SPECT RNA may be in the best position to further develop wall motion analysis as it provides an accurate $3 \mathrm{D}$ representation of the heart's chambers.

\subsection{Quantifying dyssynchrony}

Several parameters have been proposed and evaluated for MD quantification, yet no single parameter has been widely adopted for clinical application. In FA, several parameters have been investigated (see section 2.2.1) and produced promising results at quantifying MD and predicting CRT response. However, there are several unexplored directions that are needed to better analyze wall motion.

Firstly, to date, mostly global FA parameters have been investigated for MD quantification and the potential of segmental FA remains undetermined. Several scar studies (described in section 2.3) have remarked that segmental LV analysis (i.e. lateral wall) produced the best results for predicting response to CRT. Current global MD FA parameters may be diluting significant results that segmental analysis may provide. There is a need to investigate FA parameters in different LV segments. In this study, a 5 
segment model, as defined in 2.1.4.1 and Figure 2.5 is adopted to investigate segmental analysis.

Secondly, FA parameters have almost exclusively focused on phase analysis, while amplitude analysis been largely ignored despite being derived in the first order fit. Synchrony is one of the few MD parameters that incorporates amplitude values and has shown potential as a MD parameter $(68,73)$. This suggests that amplitude may provide additional information and there is a need to investigate the value of amplitude parameters obtained in first order FA.

Thirdly, the TACs analyzed in FA are not perfect sinusoidal curves and therefore a first

order Fourier fit that is commonly used in FA is not representative of the true shape of the curve. This may lead to the loss of valuable information concerning wall motion. A novel technique which analyzes the full shape of the TACs may provide additional information not provided by FA. Cluster analysis techniques may offer advantages in this regard and will be described further in chapter 5.

\subsection{Study Populations}

In this thesis, three patient populations are used for analysis: a normal cardiac population and two diseased populations: PREDICT and RAFT. 


\subsubsection{Normal cardiac population}

The normal cardiac population consists of 50 subjects who have undergone SPECT RNA scans for baseline cardiotoxicity assessment prior to undergoing chemotherapy. All 50 patients have normal electrical and mechanical cardiac function (LVEF $>50 \%$, QRS $<120$ $\mathrm{ms}$ ). This population is used in chapter 5 to define normal cardiac wall motion TACs.

\subsubsection{PREDICT population}

The "PET to assess reduction in end systolic volume following CRT" (PREDICT) population (1) consists of $49 \mathrm{CHF}$ patients selected for CRT (LVEF $<30 \%$, QRS $>120$ ms, NYHA class II or III) who were referred to the University of Ottawa Heart Institute (UOHI). This group received both a planar and SPECT RNA scan, as well as PET perfusion and viability scans prior to CRT. All patients underwent RNA scans 3 months after CRT for functional improvement assessment. All patients filled written consent forms and the PREDICT study was approved by the University of Ottawa Research Ethics Board. It is used in chapter 3 to investigate the correlation between amplitude parameters and the presence of scar. In chapter 4, the population is used to identify the best phase and amplitude parameters which predict CRT outcome. Finally, in chapter 5, the PREDICT population serves to optimize the cluster analysis algorithm for CRT prediction outcome.

\subsubsection{RAFT Population}

The Resynchronization-Defibrillation for Ambulatory Heart Failure Trial (RAFT) population $(2,3)$ is a large, multi-centre study spearheaded by the UOHI, which enrolled 
nearly 1800 patients CHF patients with LVEF $\leq 30 \%$, QRS $\geq 120 \mathrm{~ms}$, NYHA class II/III. The study is randomized trial which investigated the benefits, if any, of adding CRT to an ICD in mild to moderate CHF patients. A sub-population $(\mathrm{N}=214)$ of the RAFT group underwent SPECT RNA scans prior to undergoing surgery for an ICD or ICD and CRT. This population is used in chapter 4 to further evaluate the best predictive parameters identified by the PREDICT population.

\subsection{Objective and Hypothesis}

The goal of this research is to enhance the quantification of cardiac wall motion using novel SPECT RNA methodologies for improved mechanical dyssynchrony evaluation of patients. Using the baseline scans of patients selected for CRT, the following hypotheses will be tested:

An improvement in the assessment of mechanical dyssynchrony will help predict the outcome of CRT patients alone. The incorporation of segmental mechanical dyssynchrony parameters will provide valuable information that may be lost or diminished in single, global dyssynchrony parameters. Novel amplitude based parameters obtained from FA will correlate with scar and provide valuable information

that may be used as a surrogate for predicting CRT response. Lastly, cluster analysis will provide a novel way to assess mechanical dyssynchrony patterns which may improve on existing FA techniques to predict CRT response because it includes the full shape of the TACs in its analysis. 


\section{Chapter 3}

\section{Evaluation of the correlation between scar}

\section{and amplitude}

\subsection{Introduction}

Myocardial scar size in the lateral wall has been shown to be predictive of CRT response (1). It has not been previously verified whether FA parameters and scar values are independent or if there exists a degree of interrelation between them. Scar is associated with weakened contractile function which impairs regional LV wall motion. A reduction in the magnitude of contraction in a scarred segment may cause a reduction in the corresponding amplitude values derived from FA. Thus the size of the myocardial region with abnormal amplitude, as obtained from FA, may correlate with scar size and could serve as a surrogate to scar size for predicting CRT response. The two main objectives of this study were to determine whether novel amplitude parameters could serve as a surrogate marker for scar in predicting response to CRT and, secondly, to determine the correlation between amplitude in the lateral wall as assessed by SPECT RNA and lateral wall scar as assessed by FDG PET imaging. 


\subsection{Material and Methods}

\subsubsection{Patients}

The PREDICT population (section 2.5.2) was used for this analysis which included 49 patients (LV ejection fraction $(\mathrm{LVEF})<30 \%$, QRS $>120 \mathrm{~ms}$, New York Heart Association (NYHA) class II or III) who underwent baseline PET scans as well as a SPECT RNA scan prior to pacemaker implantation. Of the 49 patients, 39 had left bundle branch block (LBBB) and 10 were diagnosed with another non-specific LV intraventricular conduction delay.

The patient population was further stratified into subgroups of ischemic $(n=27)$ and nonischemic $(\mathrm{n}=22)$ etiology as follows. Patients with a history of myocardial infarction and evidence of coronary disease (at least 1 stenosis $\geq 70 \%$ in at least 2 major arteries) were classified as ischemic. Patients without history of infarction or coronary disease were classified non-ischemic $(1,126)$.

\subsubsection{Scar / Amplitude Data Acquisition}

\subsubsection{PET Imaging}

Each patient underwent both a Rubidium-82 PET perfusion scan and a F-18 fluorodeoxyglucose (FDG) viability PET scan under resting conditions. PET studies were performed on a Discovery Rx PET/CT scanner (GE Healthcare) following our standard clinical protocols (127-130). Briefly, a low-dose CT transmission scan was performed first for attenuation correction. Intravenous doses of approximately 10 
$\mathrm{MBq} / \mathrm{kg}$ of $\mathrm{Rb}-82$ and $5 \mathrm{MBq} / \mathrm{kg} \mathrm{F}-18$ FDG were then administered for the perfusion (8 min) and viability (30 min) scans respectively.

\subsection{PET Analysis}

Both PET scans were used in conjunction to assess the extent of scar in the LV, following methods established in our lab $(128,131)$. Transverse images were reconstructed using Fourier rebinning and filtered back projection with $12 \mathrm{~mm}$ Hann window of the Ramp filter, and then analyzed automatically by the FlowQuant $(\mathrm{C}$ software $(127,128,132,133)$ (University of Ottawa Heart Institute). In brief, the LV was sampled into a polar map with 460 sectors. Following the automatic normalization and scaling process $(127,128)$, each sector was assigned a perfusion value equal to its percentage of maximum uptake. Any sector with less than $80 \%$ normalized perfusion and less than $80 \%$ normalized FDG uptake was considered to have scar $(127,128)$. Scar size was defined as the number of sectors (expressed as a percent of the LV) which fit these criteria.

For each scar sector, a scar score was calculated (equation 3.1) by subtracting the normalized FDG (or perfusion value, whichever is greater) from the maximum normalized perfusion value, (i.e. 1 - FDG) (128). Total scar score was calculated by summing the scar scores across all the sectors in the desired segment, dividing by the number of sectors and multiplying by 100 to obtain a percent score.

$$
\operatorname{Scar} \operatorname{Score}(\%)=100 * \sum_{i=1}^{n} \frac{\left(1-F D G_{i}\right)}{n}
$$


where $F D G_{i}$ is the normalized uptake of FDG in the ith sector and $n$ is the number of polar map sectors in the lateral wall. The lateral wall was defined based on a 5 segment heart model, as shown in Figure 2.5.

\subsubsection{RNA Imaging}

Prior to the RNA scan, patients were administered $1417 \pm 212 \mathrm{MBq}$ of Tc-pertechnetate using a modified in vivo red blood cell labeling method (see section 2.1.2). A planar RNA scan was acquired at the $45^{\circ}$ left anterior oblique orientation and electrocardiogram (ECG)-gated into 24 time frames. SPECT RNA imaging was then performed using a dual head gamma camera $\left(90^{\circ}\right.$ orientation) with low energy high resolution collimator (CardiaL gamma camera (GE Healthcare)). Counts were recorded at 60 different projections for 25 seconds per projection over a span of $180^{\circ}$ and were ECG-gated to 8 time frames.

The SPECT data were reconstructed into transverse slices using filtered back projection. Images were smoothed using a fifth order 3D Butterworth filter (0.35 Nyquist frequency cutoff), reoriented to short axis slices and resized from 64 X 64 to 128 X 128 using cubicspline interpolation for a final pixel size of $3.44 \mathrm{~mm}$ X $3.44 \mathrm{~mm}$ X $6.88 \mathrm{~mm}$. 


\subsection{SPECT RNA Fourier Analysis}

SPECT RNA data were analyzed using in-house FA software described in section 2.2.2. For each patient, all FA amplitude values were normalized to the patient's maximum amplitude. This reduced the inter-patient variability by minimizing the effect of factors such as the amount of activity administered and allowed for more accurate comparisons between patients.

For this analysis, an amplitude size and an amplitude score parameter were developed analogous to scar size and scar score definitions. A sector was defined as abnormal if its normalized amplitude was less than a certain threshold. To determine the amplitude threshold which would produce the best prediction of CRT response, cut-off values from $10 \%$ to $90 \%$ in increments of $1 \%$ were investigated. Abnormal amplitude size (AmpSize) was defined as the number of sectors, expressed as a percentage, which had abnormal amplitude values.

For each abnormal sector, an abnormal amplitude score (equation 3.2) was calculated by subtracting the sector's normalized amplitude value from 1, (i.e. 1 - amplitude). Total abnormal amplitude score (AmpScore) was calculated by summing the amplitude scores across all the sectors in the lateral wall, dividing by the number of sectors and multiplying by 100 to obtain a percent score.

$$
\operatorname{AmpScore}(\%)=100 * \sum_{i=1}^{n} \frac{\left(1-\text { amplitude }_{i}\right)}{n}
$$


where amplitude $e_{i}$ is the normalized amplitude in the $i^{\text {th }}$ segment and $n$ is the number of sectors in the lateral wall. The lateral wall was defined based on a 5 segment heart model, as shown in Figure 2.5.

\subsubsection{CRT Response}

Response to CRT was based on reverse remodeling of the left ventricle assessed at a 3month post-implant follow-up. At the 3 month follow-up, both planar and SPECT RNA scans were acquired following the same protocols used for the pre-implant acquisitions. The pre-defined response criteria were either an improvement in LVEF $\geq 5 \%$ and/or a reduction (improvement) in LV end-systolic volume (LVESV) by $10 \%$ or more $(1,36)$. Planar RNA data were used to calculate LV ejection fraction as well as end-systolic and end-diastolic volumes using FUGA (FUnctional Gated Analysis) software from Hermes Medical Solutions. LV segmentation was performed automatically by FUGA software using a second order derivative technique established by Forrester et al. (134) in which the segmented LV region of interest (ROI) obtained at end-diastole was fixed across each frame. The total counts in the ROI obtained at end-diastolic and end-systolic frames are individually corrected for background counts and then used to calculate the LV ejection fraction. End-systolic and end-diastolic volumes were then calculated using the technique defined by Massardo (135).

\subsubsection{Data Analysis}

A Pearson correlation coefficient ( $r$ ) was used to determine the correlation between AmpSize and scar size, as well as AmpScore and scar score, in the lateral wall. A 
Wilcoxon rank sum test was used to determine if any significant differences existed between the means of two sample data sets. This test was performed on the whole population as well as in the two subgroups: ischemic and non-ischemic. A Fisher exact test was used for comparisons of categorical variables and a Fisher z-transform was used

to compare the Pearson correlation coefficients. A p-value of $\leq 0.05$ was considered statistically significant for all tests.

ROC AUC values were computed using ROCKIT software (University of Chicago, IL) to determine the ability of FA parameters to predict response to CRT. Cut-off values were chosen so that the specificity would be similar to those presented in the Birnie paper (1) for scar size. Sensitivity, specificity and cut-off value were reported for each parameter.

\subsection{Results}

\subsubsection{Patients}

Of the 49 patients analyzed, 31 (63\%) were identified as responders compared to 18 nonresponders. In the ischemic subgroup $(\mathrm{N}=27), 18(65 \%)$ were responders, whereas in the non-ischemic subgroup $(\mathrm{N}=22), 13(59 \%)$ were identified as responders.

Patient demographics stratified by responder status and divided in sub-groups of ischemic and non-ischemic etiology are shown in Table 3.1 and Table 3.2. None of the baseline variables were significantly different $(\mathrm{p}>0.05)$ between responders and non-responders in any group. 
Table 3.1 Patient demographics for the PREDICT population stratified by responder status

\begin{tabular}{|c|c|c|c|}
\hline & \multicolumn{2}{|c|}{ Responders } & Non-Responders \\
\hline $\mathrm{N}$ & \multicolumn{2}{|l|}{31} & 18 \\
\hline Age & \multicolumn{2}{|l|}{$68 \pm 9$} & $67 \pm 10$ \\
\hline LVEF $(\%)$ & \multicolumn{2}{|l|}{$21 \pm 7$} & $23 \pm 8$ \\
\hline LVESV (mL) & \multicolumn{2}{|l|}{$232 \pm 77$} & $231 \pm 136$ \\
\hline QRS duration (ms) & \multicolumn{2}{|l|}{$172 \pm 25$} & $162 \pm 27$ \\
\hline Gender $(\mathrm{M} / \mathrm{F})$ & \multicolumn{2}{|l|}{$24 / 7$} & $17 / 1$ \\
\hline NYHA Class (II/III) & \multicolumn{2}{|l|}{$10 / 21$} & $3 / 15$ \\
\hline$\Delta \mathrm{EF}(\%)$ & $10 \pm 9$ & * & $-1 \pm 5$ \\
\hline$\Delta \mathrm{ESV}(\%)$ & $-30 \pm 23$ & * & $11 \pm 26$ \\
\hline \multicolumn{4}{|c|}{$\mathrm{LVEF}=$ left ventricular ejection fraction; LVESV = left ventricular } \\
\hline
\end{tabular}


Table 3.2: Patient demographics stratified by ischemic etiology and responder status

\begin{tabular}{|c|c|c|c|c|c|c|}
\hline & \multicolumn{3}{|c|}{ Ischemic population } & \multicolumn{3}{|c|}{ Non-Ischemic population } \\
\hline & \multicolumn{2}{|c|}{ Responders } & Non-Resp. & \multicolumn{2}{|c|}{ Responders } & Non-Resp. \\
\hline $\mathrm{N}$ & \multicolumn{2}{|l|}{18} & 9 & \multicolumn{2}{|l|}{13} & 9 \\
\hline Age & \multicolumn{2}{|l|}{$73 \pm 7$} & $70 \pm 8$ & \multicolumn{2}{|l|}{$62 \pm 8$} & $64 \pm 11$ \\
\hline LVEF (\%) & \multicolumn{2}{|l|}{$20 \pm 6$} & $25 \pm 5$ & \multicolumn{2}{|l|}{$22 \pm 8$} & $21 \pm 11$ \\
\hline LVESV (mL) & \multicolumn{2}{|l|}{$227 \pm 68$} & $186 \pm 51$ & \multicolumn{2}{|l|}{$239 \pm 90$} & $278 \pm 179$ \\
\hline QRS duration (ms) & \multicolumn{2}{|l|}{$173 \pm 25$} & $154 \pm 30$ & \multicolumn{2}{|l|}{$169 \pm 25$} & $171 \pm 22$ \\
\hline Gender (M/F) & \multicolumn{2}{|l|}{$17 / 1$} & $9 / 0$ & \multicolumn{2}{|l|}{$7 / 6$} & $8 / 1$ \\
\hline NYHA Class (II/III) & \multicolumn{2}{|l|}{$6 / 12$} & $3 / 6$ & \multicolumn{2}{|l|}{$4 / 9$} & $0 / 9$ \\
\hline$\Delta \mathrm{EF}(\%)$ & $7 \pm 7$ & $*$ & $3 \pm 11$ & $14 \pm 10$ & $*$ & $-2 \pm 6$ \\
\hline$\Delta \mathrm{ESV}(\%)$ & $-22 \pm 23$ & $*$ & $20 \pm 35$ & $-39 \pm 21$ & $*$ & $20 \pm 35$ \\
\hline $\mathrm{LVEF}=$ left ventricular ejecti & raction; LVES & & ventricular end- & -systolic volur & ;NY & $\mathrm{IA}=\mathrm{New}$ \\
\hline
\end{tabular}

\subsubsection{Scar vs. Amplitude Correlation}

Correlation results between lateral wall scar and amplitude size are shown in Table 3.3 and Figure 3.1, whereas correlation between lateral wall scar and amplitude score are shown in Table 3.4 and Figure 3.2. 
A

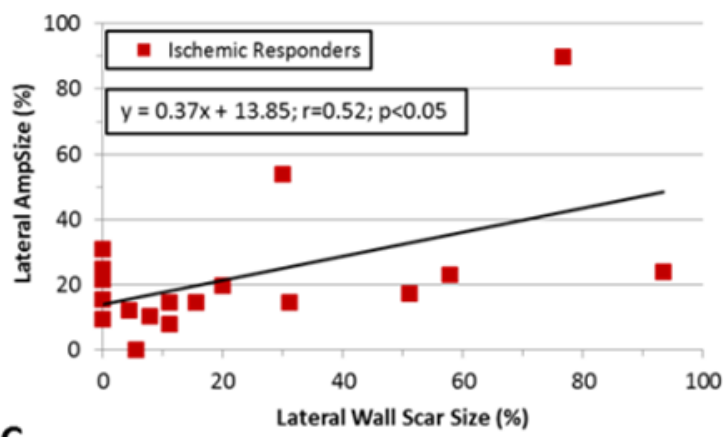

C

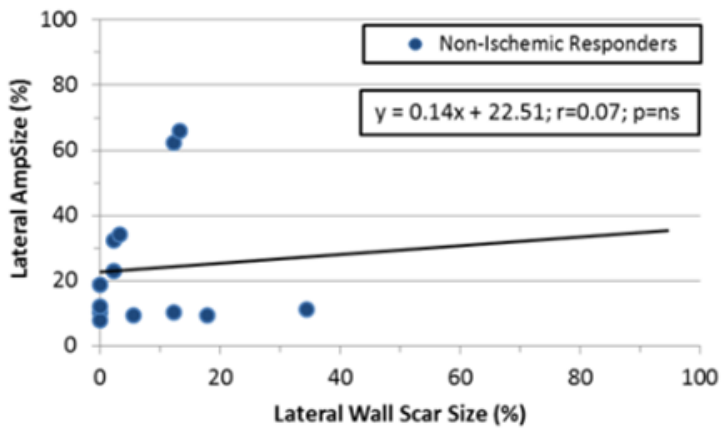

B
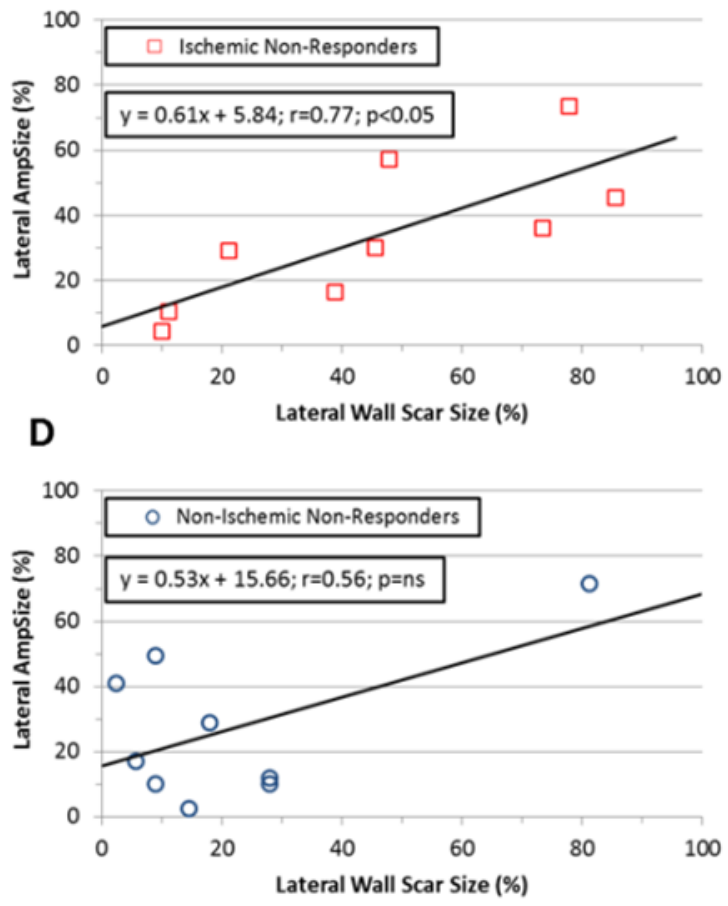

Figure 3.1: Correlation between lateral AmpSize and lateral wall scar size for: $A$ ) ischemic responders, B) ischemic non-responders, C) non-ischemic responders and D) non-ischemic nonresponders

Table 3.3: Correlation coefficient between AmpSize and scar size in the lateral wall

\begin{tabular}{cccc}
\hline Population & $\mathrm{r}$ & Population & $\mathrm{r}$ \\
\hline Ischemics & $0.64^{*}$ & Ischemic Responders & $0.52^{*}$ \\
& & Ischemic Non-Responders & $0.77^{*}$ \\
Non-Ischemics & 0.36 & Non-Ischemic Responders & 0.07 \\
& & Non-Ischemic Non-Responders & 0.56 \\
Whole & $0.51 *$ & & \\
\hline * statistically significant $(\mathrm{p} \leq 0.05)$ & &
\end{tabular}


A

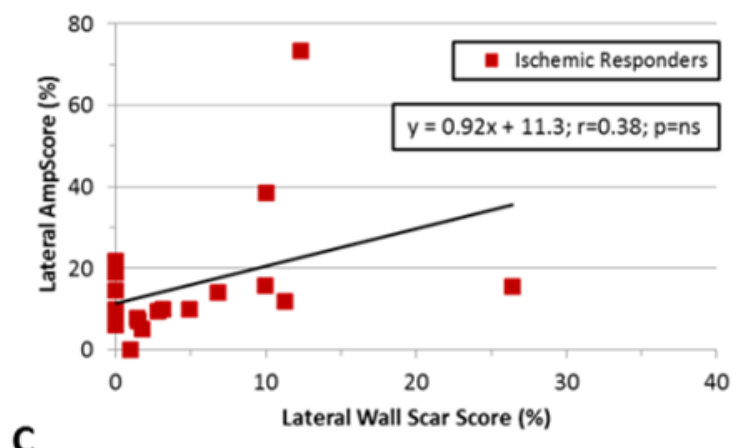

C

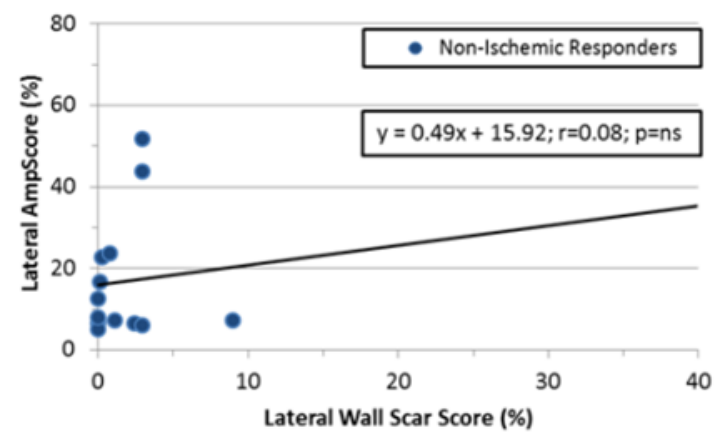

B
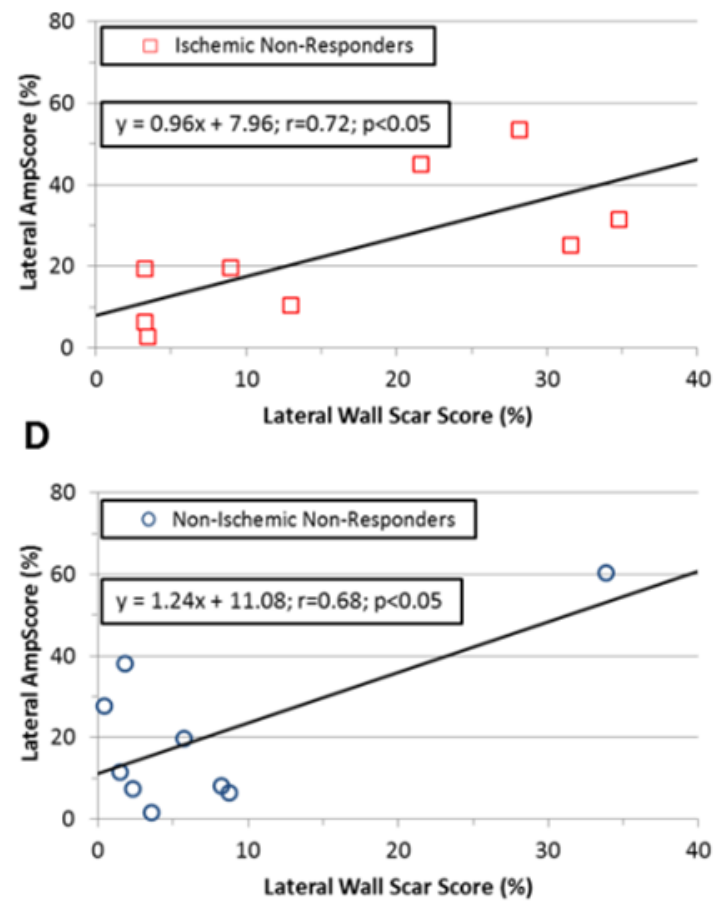

Figure 3.2: Correlation between lateral AmpScore and lateral wall scar score for: A) ischemic responders, B) ischemic non-responders, C) non-ischemic responders and D) non-ischemic nonresponders

Table 3.4: Correlation coefficient between AmpScore and scar score in the lateral wall

\begin{tabular}{llll}
\hline Population & $\mathrm{r}$ & Population & $\mathrm{r}$ \\
\hline Ischemic & $0.55^{*}$ & Ischemic Responders & 0.38 \\
& & Ischemic Non-Responders & $0.72^{*}$ \\
Non-Ischemic & $0.47^{*}$ & Non-Ischemic Responders & 0.08 \\
& & Non-Ischemic Non-Responders & $0.68^{*}$ \\
Whole & $0.50 *$ & & \\
& & &
\end{tabular}


Lateral wall AmpSize and AmpScore showed a significant positive correlation $(\mathrm{p} \leq 0.05)$ with lateral wall scar size $(r=0.51)$ and lateral wall scar score $(r=0.50)$, respectively, in the whole patient cohort. In both cases, the correlation was further strengthened in the ischemic sub-population $(\mathrm{r}=0.64$ and $\mathrm{r}=0.55)$ for AmpSize and AmpScore respectively $(\mathrm{p} \leq 0.05)$

All subset combinations of ischemia/non-ischemia and responders/non-responders showed positive correlation between lateral wall AmpSize and lateral wall scar size, though only ischemic responders and ischemic non-responder sub-groups were significant $(\mathrm{p} \leq 0.05)$. The correlation between AmpScore and scar score was significant $(\mathrm{p} \leq 0.05)$ in the ischemic non-responder group as well as in the non-ischemic nonresponder sub-group.

\subsubsection{CRT Response}

An amplitude threshold of $41 \%$ was determined to give the best AmpSize and AmpScore results for ROC analysis. All amplitude-based results presented in this study were obtained using a $41 \%$ cutoff. Table 3.5 shows ROC AUC, and the chosen sensitivity and specificity for AmpSize, AmpScar, scar size and scar score in the lateral wall. ROC AUC results indicated that scar-based parameters were predictive of CRT outcome $(\mathrm{p} \leq 0.05)$

Scar-based parameters tended to produce greater ROC AUC values than amplitude-based parameters but the differences were not statistically significant in any of the groups $(p>$ 
0.05). ROC AUC values for both scar and amplitude were greater in the ischemic group for all parameters. Amplitude based parameters had a sensitivity of $83 \%$ and specificity of $67 \%$ in the ischemic population, while scar size had a sensitivity of $78 \%$ and specificity of $67 \%$, and scar score had $72 \%$ sensitivity and $67 \%$ specificity at the chosen cutoff values. Figure 3.3 compares the ROC curves of amplitude and scar the ischemic population. However, in the non-ischemic population, scar size results suggest improved performance over the other amplitude parameters, producing a sensitivity of $69 \%$ and specificity of $78 \%$.

Table 3.5: Lateral wall ROC area under the curve values for CRT response prediction for AmpSize, AmpScore, scar size and scar score for each group. Comparable sensitivity, specificity and cut-off values are also presented. Scar size data previously published by Birnie et al. (1).

\begin{tabular}{lllll}
\hline Population & Amp Size & Amp Score & Scar Size & Scar Score \\
\hline Whole Population & $0.60 \pm 0.08$ & $0.60 \pm 0.08$ & $0.72 \pm 0.07^{*}$ & $0.78 \pm 0.07^{*}$ \\
AUC & 77 & 77 & 74 & 77 \\
Sensitivity (\%) & 76 & 56 & 56 \\
Specificity (\%) & 56 & 56 & $19.0 \%$ & $5.6 \%$ \\
Cut-off & $29.0 \%$ & $19.0 \%$ & & \\
\hline Ischemic Population & & & & \\
AUC & $0.68 \pm 0.11$ & $0.67 \pm 0.11$ & $0.75 \pm 0.10^{*}$ & $0.83 \pm 0.08^{*}$ \\
Sensitivity (\%) & 83 & 83 & 78 & 72 \\
Specificity (\%) & 67 & 67 & 67 & 67 \\
Cut-off & $25.0 \%$ & $19.0 \%$ & $31.2 \%$ & $8.4 \%$ \\
\hline
\end{tabular}




\begin{tabular}{lllll}
\hline \multicolumn{2}{l}{ Non-Ischemic Population } & & & \\
AUC & $0.56 \pm 0.13$ & $0.58 \pm 0.12$ & $0.75 \pm 0.10^{*}$ & $0.78 \pm 0.10^{*}$ \\
Sensitivity (\%) & 54 & 54 & 69 & 62 \\
Specificity (\%) & 56 & 56 & 78 & 67 \\
Cut-off & $12.5 \%$ & $10.0 \%$ & $7.0 \%$ & $2.0 \%$
\end{tabular}

AUC , area under the curve; ${ }^{*}$ ROC AUC values statistically significantly $(\mathrm{p} \leq 0.05)$
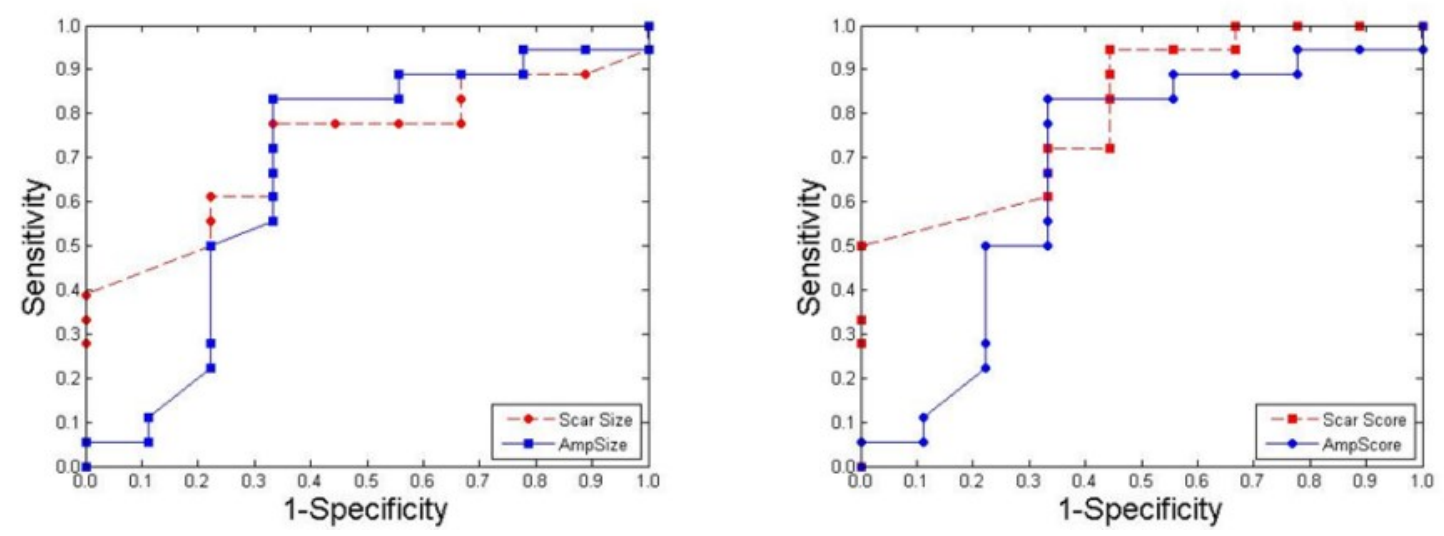

Figure 3.3: ROC Curves for a) Scar size and AmpSize and b) Scar Score and AmpScore in the lateral wall for the ischemic population. ROC AUC values for AmpSize and AmpScore were 0.67 and 0.68 respectively ( $p=n s)$ while ROC AUC values of 0.75 and $0.83(p \leq 0.05)$ were obtained for scar size and score respectively. None of the AUC values were significantly different from each other $(p>0.05)$

\subsection{Discussion}

A moderate positive correlation was observed between lateral wall amplitude based parameters obtained from SPECT RNA FA and the corresponding lateral wall scar parameters as assessed from perfusion and FDG PET scans. By definition, scar size and AmpSize were binary evaluators of scar, identifying sectors of low perfusion/viability or low amplitude (i.e. extent or spread of abnormal values), but they did not provide 
information on the severity of the abnormality in those regions. On the other hand, scar score and AmpScore indicated the total amount of perfusion/viability or amplitude present (i.e. severity of the abnormal value) in the lateral wall, but provided no information on the size of the region affected.

Both AmpSize and AmpScore produced similar correlation coefficients with respect to scar size and scar score which were not statistically different ( $p>0.05$ ) from one another. Although there is considerable variability in individual sector scores, once the total score sum is obtained from all sectors in the lateral wall, there exists a strong correlation between score and size for both amplitude and scar based parameters. This suggests that the average score per sector is relatively constant across patients.

To separate normal from abnormal normalized amplitude, a range of cut-off values between 10 and $90 \%$ were investigated. It was determined that a cut-off of $41 \%$ produced the largest ROC AUC for predicting response to CRT in the PREDICT population for the AmpSize and AmpScore metrics. In another population, a different abnormal amplitude threshold value may provide improved ROC AUC results as the size of the PREDICT population was relatively small and thus may not represent the general population. Nevertheless, the ROC AUC values were not very sensitive to the choice of cut-off as similar AUC values were found for a wide range of cut-off values. Additionally, changing the threshold cut-off value of AmpSize and AmpScore may also impact the correlation with scar parameters. However, the impact is believed to be minimal as the direct correlation of the normalized amplitude (without using any cut-off 
values) to the scar-based parameters varied little from the ones presented in Tables 3.3 and 3.4. The correlation differences were all less than 0.06 and none were significant.

Using lateral wall scar size as a measure of scar, a correlation coefficient of $r=0.51$ was observed when comparing to lateral wall AmpSize. This correlation was further strengthened when investigating the ischemic population exclusively $(\mathrm{r}=0.64)$ but was not significant in the non-ischemic population $(\mathrm{r}=0.36)$. It was expected that ischemia would induce necrosis in myocardial tissue which would be observed as scar and decreased contractility, leading to reduced wall motion and thus greater AmpSize. Ischemic patients presented a greater range of wall motion abnormalities relative to nonischemic patients which may have helped improve correlation coefficients. Furthermore, scar formation in ischemic cardiomyopathy patients is characteristically transmural scar, whereas for non-ischemic cardiomyopathy patients the mechanism of scar formation is not as clear and is often restricted to the epicardium and midwall (136-138). These differences in scar formation and location could explain differences in significance of the correlation values between the two sub-groups. With scar score and AmpScore, where the severity of scar or amplitude reduction is included in the metric, the results are similar for the two sub-populations. The correlation in the lateral wall was found to be significant in both sub-populations: $\mathrm{r}=0.55$ in the ischemic population and $\mathrm{r}=0.47$ in the non-ischemic population.

The moderate correlation results suggest that there is some independence between the two metrics, and that factors other than scar impact LV contraction amplitude. This is 
also demonstrated by the wide spread of amplitude values observed in patients with low scar sizes, as shown in Figure 3.1.

Non-ischemic cardiomyopathy patients form a heterogeneous group in which heart failure can be attributed to a variety of causes that can affect the myocardium differently. This may cause greater variability in the magnitude of contraction of the LV unrelated to the presence of scar. Furthermore, the different location and mechanisms of scar formation due to the various types of non-ischemic cardiomyopathy may also affect the contraction of the heart differently. Both of these factors might alter the correlation between amplitude and scar in non-ischemic populations.

Lateral wall scar score produced the greatest ROC AUCs of all parameters: 0.78 in the whole population, 0.83 and 0.78 in the ischemic and non-ischemic populations respectively ( $\mathrm{p} \leq 0.05$ compared to chance). Previously, our group identified that lateral wall scar size was shown to be predictive of CRT outcome $(\mathrm{p} \leq 0.05)$ in this same study population, and ROC values were found to be $0.72,0.75$ and 0.75 in the whole, ischemic and non-ischemic populations respectively (1). In all cases, values below the cutoff denoted the responder group, indicating that CRT recipients are more likely to respond if they have a low amount of lateral wall scar or a low lateral wall AmpSize/AmpScore value (i.e. patient possesses normal amplitude).

While ROC AUC values for AmpSize and AmpScore did not achieve statistical significance $(p>0.05)$ with respect to chance, ROC AUC and the optimal values of 
sensitivity and specificity were not significantly different $(p>0.05)$ in both the whole population and ischemic population groups from those of scar size and scar score. Furthermore, AmpSize and AmpScore successfully identified 3 responders and 1 nonresponder that both scar based parameters incorrectly identified in the ischemic population whereas scar size correctly identified 2 responders and 1 non-responder in contrast to AmpSize and AmpScore. This fact, combined with similar ROC results and the only moderate correlation results of amplitude-based parameters versus scar-based parameters in the ischemic population suggest that amplitude-based parameters may be imperfect surrogate markers of scar but may provide additional complementary information to scar-based parameters for detecting CRT response.

Responders had significantly less lateral wall scar than non-responders and all patients $(\mathrm{N}=9)$ without lateral wall scar were responders. Furthermore, 16 of the 20 patients with lateral scar less than $10 \%$ were responders, confirming that a lack of scar or minimal scar is highly indicative of response. However, the variability of lateral wall amplitude values observed in these same low scar patients was large, as observed in Figure 3.1 and Figure 3.2, causing a large overlap of lateral wall AmpSize (AmpScore) values between the responder and non-responder groups and reducing the statistical significance of lateral wall amplitude parameters as a predictor of CRT. As can be observed in Figure 3.3 for the ischemic population, at low scar values $(\sim<12 \%)$, scar size (score) provides excellent specificity, but due to the large variability in amplitude values for patients who possess low scar, the ROC curve for AmpSize (AmpScore) performed considerably worst. For higher scar values $(>12 \%)$, both amplitude based and scar based parameters performed 
equivalently. This explains the smaller ROC AUC values reported for lateral wall AmpSize and AmpScore despite the equivalence of the selected sensitivity and specificity values with respect to scar size and score.

A limitation of the study is that the polar maps were generated independently for the PET and SPECT RNA scans and thus may not have been perfectly aligned. A misregistration could cause some amplitude values to be assigned to a different segment than the scar value of that sector. However, the polar maps were split into 5 large segments and misregistration of a few sectors would have limited impact on total value measured in the lateral wall. Furthermore, the cutoff values of $80 \%$ normalized perfusion and $80 \%$ normalized FDG uptake, used to denote the presence of scar, are specific to our institution. Different cutoff values are possible and the effect of varying these values is unclear and is beyond the scope of this study.

A further limitation of the SPECT RNA FA is that the ROI's positioned around the LV wall are fixed as the LV beats. Consequently, all types of cardiac wall motion (contraction, rotation, longitudinal motion) will affect the counts recorded in an ROI. However, global translation and rotation of the LV wall may be independent of regional scar. For example, it is possible for a section of the LV to not be contracting due to presence of scar, but for the counts in a fixed ROI to change due to a translation of the LV. Further investigation is warranted to establish the magnitude of effect that rotational and translational heart motion have on measured amplitudes. 
The relatively small population size limited the statistical power for determining significant differences in amplitude between responders and non-responders, especially when subdividing into ischemic and non-ischemic groups. However, the population size was sufficiently large to establish a correlation between amplitude and scar in most subgroups.

This study is the first to examine the correlation between scar and magnitude of contraction as assessed by amplitude analysis. In the ischemic population, amplitude based parameters provided similar sensitivity and specificity to that determined using scar values. However the correlation was only moderate and different specific responders / non-responders were identified with the two metrics. This suggests that amplitude should be further developed and utilized in FA to better quantify mechanical dyssynchrony, and consequently predict CRT outcome. Three MRI studies have reported that adding a dyssynchrony marker provides incremental information to scar for the prediction of CRT response $(120,123,139)$. A recent SPECT myocardial perfusion imaging study showed that combining mechanical dyssynchrony, myocardial scar and site of latest activation resulted in a positive predictive value of $>90 \%$ for identifying CRT responders (140). All of these results suggest that a combination of several parameters may be required to be able to significantly improve the ability to predict response to CRT. Further investigation is warranted to determine if a combination of scar size and a mechanical dyssynchrony FA parameter may improve patient selection for CRT. 


\subsection{Conclusion}

Lateral wall amplitude based parameters obtained from SPECT RNA FA produced an overall accuracy in predicting CRT response in ischemic patients that was not significantly different to that predicted by PET lateral wall scar parameters. A significant correlation existed between amplitude size and scar size in the lateral wall but an $\mathrm{r}=0.51 \quad(\mathrm{r}=0.64$ for ischemic patients) suggested that amplitude may provide complementary information to scar for identifying CRT responders. 


\section{Chapter 4}

\section{Development of novel FA parameters}

\subsection{Introduction}

In the previous chapter, AmpSize and AmpScore were evaluated exclusively in the lateral wall for their ability to predict CRT response. That evaluation showed promising results and so in this chapter, the investigation of segmental wall-motion analysis was expanded to include all of the 5 segments. As discussed previously, amplitude has not been investigated to the same extent as phase-based parameters and could potentially provide valuable information on MD. Therefore, several novel amplitude-based FA parameters were developed and evaluated for their ability to predict response to CRT. The amplitude-based parameters were compared to established phase-based FA parameters in the context of a small study population (PREDICT). The most promising candidates for both phase- and amplitude-based parameters were then carried forward for evaluation in a larger blinded study (RAFT) to assess their respective abilities to predict response to CRT therapy.

Two potential confounding factors were identified as part of the previous study (chapter 3): global cardiac motion and the count drop-out in the latter time-bins due to variable R- 
R ECG gating intervals. Novel methods to incorporate compensation for these factors and thereby assess their impact were also included in the studies presented in this chapter.

\subsection{Materials and Methods}

\subsubsection{Patients}

The PREDICT study (section 2.5.2) was used to identify FA parameters that have the potential to predict response to CRT. A total of 49 patients were evaluated and a total of 27 patients were classified as ischemic, as established in chapter 3.

The RAFT study (section 2.5.3) was used to validate selected FA parameters, identified by the PREDICT population, that were significantly different between responder and non-responder groups. A total of 214 CHF patients (LVEF $\leq 30 \%$, QRS $\geq 120 \mathrm{~ms}$, NYHA class II/III) in the RAFT study received SPECT RNA scans. Of these, 103 were placed in the ICD control arm and 111 received both an ICD and CRT. The majority of the analysis focused on the ICD+CRT group because the main objective was to better understand why some CRT recipients do not respond.

\subsubsection{SPECT RNA data acquisition}

The imaging acquisition protocol used was identical, with the exception of temporal resolution, in both the RAFT and PREDICT studies and was detailed in the previous chapter (see section 3.2.2.2). 
Each SPECT RNA scan obtained for the PREDICT population was ECG gated into 8 time frames. In the RAFT population, the SPECT RNA scans of 168 patients were gated into 8 time frames while the RR interval was divided into 16 gates for the 46 remaining patients.

\subsubsection{Compensation for count drop-off}

Gating artifacts can occur in SPECT RNA images and a common artifact is for the last gates in the cycle to contain abnormally low count densities with respect to the other gates. Images are acquired by summing over several hundred cardiac cycles, thus variations in the heart beat (R-R interval) during the course of a scan can cause the data acquisition during the latter gates to be truncated prematurely. As long as the R-R interval is within the pre-defined accepted beat window range, the counts from the cardiac cycle will be included. The shorter duration of the last gates causes a reduction in the counts acquired, creating a lower intensity image in that gate.

The presence of low-count gates affects the shape of a TAC which will in turn affect the accuracy of the first order Fourier fit. Only one study has investigated the impact of gating artifacts on FA and they determined that the presence of low count gates caused an underestimation of P_SD (141). To compensate for this effect, all SPECT RNA patient scans in our study were verified for low count density gating artifacts by determining the total counts at each gate. All gates with intensity less than $90 \%$ of the patient's maximum intensity gate were scaled up to $90 \%$ while severely low count gates under $60 \%$ of maximum intensity were dropped. In patients with gating artifacts present, FA 
parameters before and after implementation of the gating correction were compared to determine if the differences were significant.

\subsubsection{Translation correction}

To compensate for global translation of the heart during cardiac contraction, a modification was made to the LV segmentation algorithm of the SPECT RNA analysis code described in 2.2.2. As was discussed previously, in each of the 18 resampled slices, the LV COM is used at the end-diastolic gate as a starting point for the LV segmentation. Each of the 568 ROI's defining the LV were then fixed as the heart beats. To correct for translation, first, the LV COM coordinates were calculated at each gate of every slice. Second, at each gate, t, a single COM value was retained from all the slices by determining the median COM. Third, at each gate, $t$, the distance between the median LV COM and the end-diastolic (gate 1) COM was calculated (Equation 4.1). Fourth, all the ROIs across all slices at gate $t$ were shifted by the rounded integer difference in COM (Equation 4.2).

$$
\operatorname{shift}(x, y)_{t, z}=\operatorname{COM}_{1, z}-\operatorname{median}\left(\operatorname{COM}_{t, z}\right),
$$

where $\mathrm{x}$ and $\mathrm{y}$ are Cartesian coordinates of the $z^{\text {th }}$ SA slice at gate $\mathrm{t}$.

$$
\text { shiftedROI } I_{i, t, z}=R O I_{i, t, z}-\operatorname{shift}(x, y)_{t, z}
$$

where $i$ represents the $i^{\text {th }}$ ROI at slice $z$ and gate $t$. 
For example, if there were 36 ROI's positioned around the LV at the $3^{\text {rd }}$ gate of the $5^{\text {th }}$ slice, each of the 36 ROI's were shifted by the same amount, based on the COM difference between the $1^{\text {st }}$ gate and the $3^{\text {rd }}$ gate.

A TAC was created for each of the 568 ROIs based on the total counts as a function of time. Since the ROI's may have shifted at any or all gates, the TAC shape will differ from the original non-translated $\mathrm{TAC}$, affecting subsequent $\mathrm{FA}$ analysis. In order to directly evaluate the effects of translation and eliminate variability, patient data were analyzed with and without translation correction in a single step.

\subsubsection{FA analysis}

Using the PREDICT population, 7 different FA parameters were investigated for their ability to predict CRT as a global value and also for each segment in a 5 segment model. Thus, a total of 42 parameters were investigated. Of the 7 parameters, 4 have been previously described in 2.2.1 : P_SD, P_BW, P_E and S. The remaining 3 were novel parameters based on amplitude analysis. They were defined analogous to phase-based parameters and are described below. As discussed in chapter 3, all amplitude values were normalized to a patient's maximum amplitude to reduce inter-patient variability.

\subsubsection{Amplitude Average (A_AVG)}

A_AVG is a straightforward but novel FA parameter that is easily calculated by determining the average of all amplitude values in a region (global or segmental). Since the values are normalized for each patient, this value is relative, which indicates that if 
the whole segment of interest is contracting poorly, the A_AVG will appear normal. This is true of all amplitude-based parameters. However, without normalization, inter-patient variability would be too great, making it difficult to detect patients with abnormal amplitude.

\subsubsection{Amplitude Standard Deviation (A_SD)}

A_SD, defined below in equation 4.3, is analogous to P_SD (Equation 2.11):

$$
A_{-} S D=\sqrt{\frac{1}{N} \sum_{i=1}^{N}\left(A_{i}-A_{-} A V G\right)^{2}} \text {, }
$$

where $\mathrm{N}$ is the number of data points in the segment. A large A_SD value indicates a wide range of magnitude of contraction exists within the various locations in a segment. Therefore, a larger A_SD value represents an increase in MD. A_BW was not investigated as it is similar in definition to A_SD and phase-analysis results have not shown a large difference between $\mathrm{P}_{-} \mathrm{SD}$ and $\mathrm{P}_{-} \mathrm{BW}(87,89,90,125)$.

\subsubsection{Amplitude Entropy (A_E)}

The equation used to calculate A_E is analogous to $P_{-} E$ (Equation 2.13) and is repeated below for convenience (equation 4.4). A_E values range from 0 (complete order) to 1 (complete disorder) and a high degree of entropy is indicative of non-uniform wall motion and represents increased MD. 
However, the probability, U(A), is based on the amplitude distribution instead of the phase distribution.

$$
\mathrm{A} \_\mathrm{E}=\frac{\sum_{i=1}^{M} U(A)_{i} \log _{2} U(A)_{i}}{\log _{2} M}
$$

where $\mathrm{M}$ is the total number of bins and $\mathrm{U}(\mathrm{A})_{i}$ is the probability of an amplitude value being placed in bin $i$. The probability, $\mathrm{U}(\mathrm{A})_{i}$, for each bin is the fraction of the total data points located in the bin. $\mathrm{P}_{-} \mathrm{E}$ was shown to be largely insensitive to bin size and thus bin size for A_E calculations was arbitrarily chosen to be 0.01 .

\subsubsection{CRT response}

Response to CRT in the PREDICT population was defined as an improvement in LVEF $>5 \%$ and/or reduction in LVESV $>10 \%$ following the procedure outline in section 3.2.3. For the larger RAFT population, CRT response was based on having no adverse outcome, defined as subsequent hospitalization or death. Time to event was also recorded to allow for advanced statistical analysis. For this analysis, hospitalization and death were individually investigated as non-response criteria but a hospitalization and/or death combination outcome was also considered.

\subsubsection{Data Analysis}

Paired or unpaired Wilcoxon rank sum tests were used, where appropriate, to identify significant differences between two continuous variables. A Fisher exact test was used for categorical variables. Translation corrected parameters $\mathrm{p}$-values were compared to non-translation corrected parameters $\mathrm{p}$-values to determine if any parameters gained or 
lost statistical significance. ROC analysis was performed on select FA parameters in the PREDICT and RAFT population and ROC AUC values were compared using a z-score test. Youden's index was used to determine the cutoff value of optimal sensitivity and specificity. In all cases, a p-value of 0.05 or less was considered significant.

\subsection{Results}

\subsubsection{Patients}

The demographics of the PREDICT population have been given previously in Table 3.1. Using the pre-defined CRT response criteria, there were 31 responders $(63 \%)$ and 18 non-responders in the whole population. None of the baseline demographic variables presented significant differences between responders and non-responders.

Of the 214 RAFT patients with SPECT RNA data, 103 were assigned to the ICD only group, while 111 received both an ICD and CRT. The patient demographics for the RAFT population receiving both ICD and CRT are given in Table 4.1 below. For both CRT non-response criteria, CHF hospitalization and death, there were 85 responders (77\%) and 26 non-responders. In the death or CHF hospitalization combination group, $69(62 \%)$ were identified as responders, while 42 had suffered at least 1 of the adverse outcomes. QRS duration was greater $(p=0.04)$ in responders in the CHF hospitalization outcome group, but not in the other outcome groups while in the death outcome group, age was a significant predictor of death $(\mathrm{p}=0.02)$. In both the death and combination group, patients with a pre-implant NYHA class of II had significantly better chance of response than class III patients $(\mathrm{p}<0.01)$. No other baseline variables were significant. 
Table 4.1: Patient demographics for the RAFT ICD+CRT population stratified by responder for various outcomes.

\begin{tabular}{|c|c|c|c|}
\hline \multicolumn{4}{|c|}{ Non-Response criteria: CHF Hospitalization } \\
\hline & Responder & Non-responder & p-value \\
\hline $\mathrm{N}$ & 85 & 26 & \\
\hline Age & $66.4 \pm 9.4$ & $63.5 \pm 9.5$ & 0.12 \\
\hline LVEF $(\%)$ & $21.7 \pm 5.5$ & $24.0 \pm 4.7$ & 0.06 \\
\hline QRS duration (ms) & $162.3 \pm 25.0$ & $151.8 \pm 29.1$ & 0.04 \\
\hline Gender $(\mathrm{M} / \mathrm{F})$ & $71 / 14$ & $23 / 3$ & 0.76 \\
\hline NYHA Class (II/III) & $67 / 18$ & $19 / 7$ & 0.60 \\
\hline \multicolumn{4}{|c|}{ Non-Response criteria: Death } \\
\hline & Responder & Non-Responder & p-value \\
\hline $\mathrm{N}$ & 85 & 26 & \\
\hline Age & $64.6 \pm 9.5$ & $69.5 \pm 8.3$ & 0.02 \\
\hline LVEF (\%) & $22.2 \pm 5.2$ & $22.2 \pm 5.9$ & 0.92 \\
\hline QRS duration (ms) & $159.6 \pm 25.6$ & $160.7 \pm 28.9$ & 0.95 \\
\hline Gender $(\mathrm{M} / \mathrm{F})$ & $73 / 12$ & $21 / 5$ & 0.54 \\
\hline NYHA Class (II/III) & $73 / 12$ & $13 / 13$ & $<\mathbf{0 . 0 0 1}$ \\
\hline \multicolumn{4}{|c|}{ Non-Response criteria: Death or CHF hospitalization } \\
\hline & Responder & Non-responder & p-value \\
\hline $\mathrm{N}$ & 69 & 42 & \\
\hline Age & $65.5 \pm 9.5$ & $66.2 \pm 9.5$ & 0.73 \\
\hline LVEF $(\%)$ & $21.8 \pm 5.4$ & $22.8 \pm 5.3$ & 0.36 \\
\hline QRS duration (ms) & $162.1 \pm 25.0$ & $156.1 \pm 28.0$ & 0.17 \\
\hline Gender $(\mathrm{M} / \mathrm{F})$ & $58 / 11$ & $36 / 6$ & 1.00 \\
\hline NYHA Class (II/III) & $60 / 9$ & $26 / 16$ & 0.004 \\
\hline
\end{tabular}




\subsubsection{SPECT RNA data acquisition}

\subsubsection{Gating correction}

In the PREDICT population, none of the patients met the criteria to require rescaling of count intensities. In total, 17 RAFT patients had at least 1 gate rescaled and 4 patients had a single gate dropped. All patients' scans that required rescaling and/or gate drops received a 16 gate ECG gated scan. Sample total normalized count curves as a function of gate are given in Figure 4.1 below.

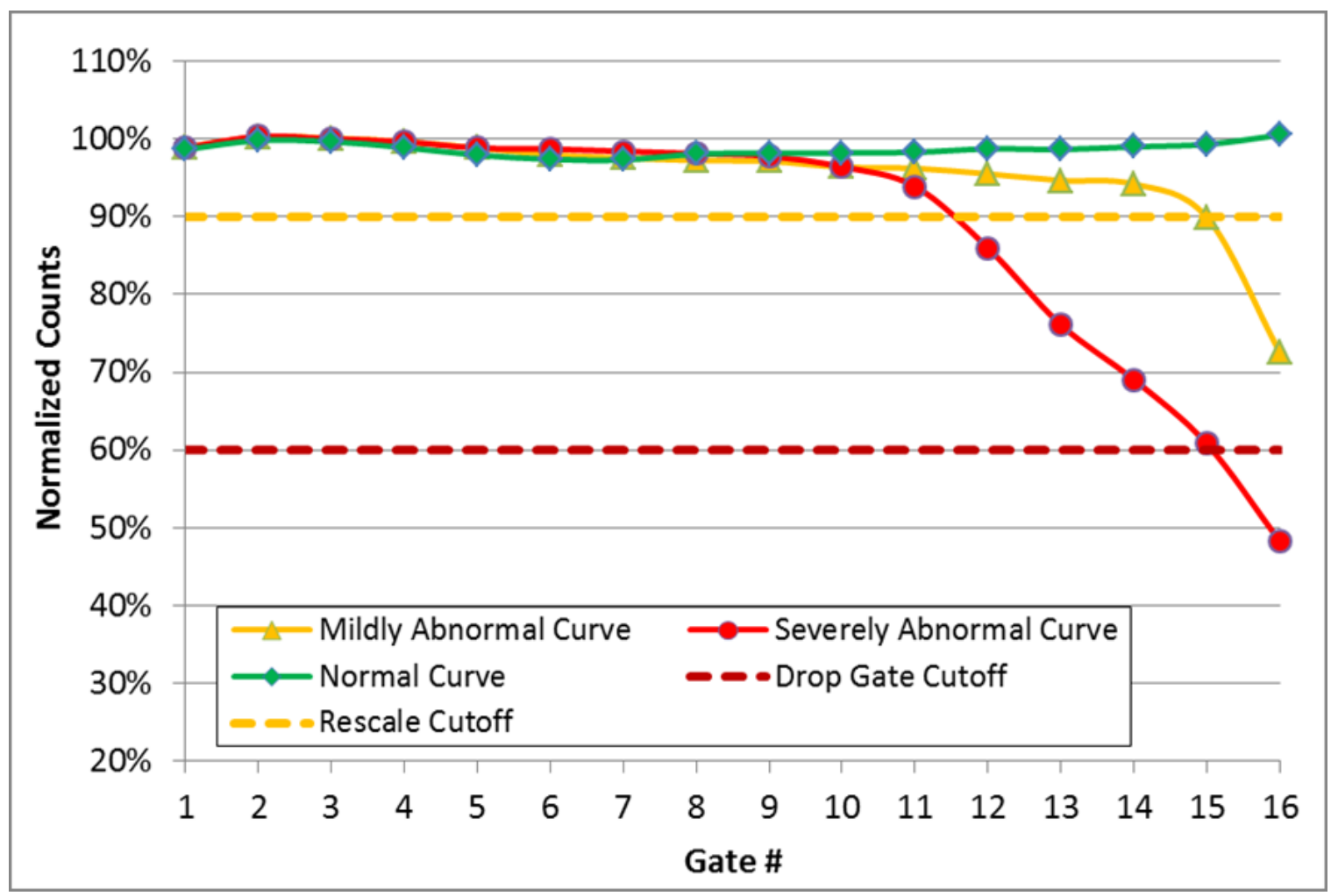

Figure 4.1: Normalized counts as a function of gate for a normal curve (green), a mildly abnormal curve requiring rescaling (yellow) and a severely abnormal curve requiring a drop gate and rescaling (red). The dashed lines represent the threshold cut-offs before rescaling (yellow) and drop gate (red) occur 
The low counts obtained in the late gates of some patients caused irregular TAC shapes and affected FA parameters. All phase-based parameters as well as S produced a lower MD value following rescaling/dropping while amplitude-based parameters produced a higher MD value, though not all values were significant (Table 4.2). Each gate of a gated SPECT acquisition image was expected to acquire approximately the same total counts; therefore a significantly lower count total must be corrected to allow for accurate analysis of the TAC shape. Once corrected, the affected TACs followed a more traditional wall motion curve shape. All patient studies affected by gating artifacts were corrected for the analysis presented in this chapter.

Table 4.2: Impact of gating artifacts corrections on FA parameters

\begin{tabular}{lccc}
\hline & Without gating correction & With gating correction & p-value \\
\hline P_SD & $60 \pm 23$ & $53 \pm 18$ & $\mathbf{0 . 0 5}$ \\
P_BW & $213 \pm 76$ & $211 \pm 83$ & 0.94 \\
P_E & $0.79 \pm 0.06$ & $0.78 \pm 0.06$ & $\mathbf{0 . 0 1}$ \\
S & $0.67 \pm 0.24$ & $0.80 \pm 0.16$ & $<\mathbf{0 . 0 0 1}$ \\
A_AVG & $0.37 \pm 0.05$ & $0.35 \pm 0.04$ & 0.09 \\
A_SD & $0.20 \pm 0.03$ & $0.21 \pm 0.03$ & 0.06 \\
A_E & $0.71 \pm 0.03$ & $0.72 \pm 0.02$ & 0.31 \\
\hline
\end{tabular}




\subsubsection{Translation correction}

On average, applying translation correction caused a shift in the negative $\mathrm{x}$-axis of a SA slice (towards the RV) of 1 pixel $(3.44 \mathrm{~mm})$, and approximately 0.5 pixels in the positive y-axis. The average shift as a function of gate is depicted in Figure 4.2 below:
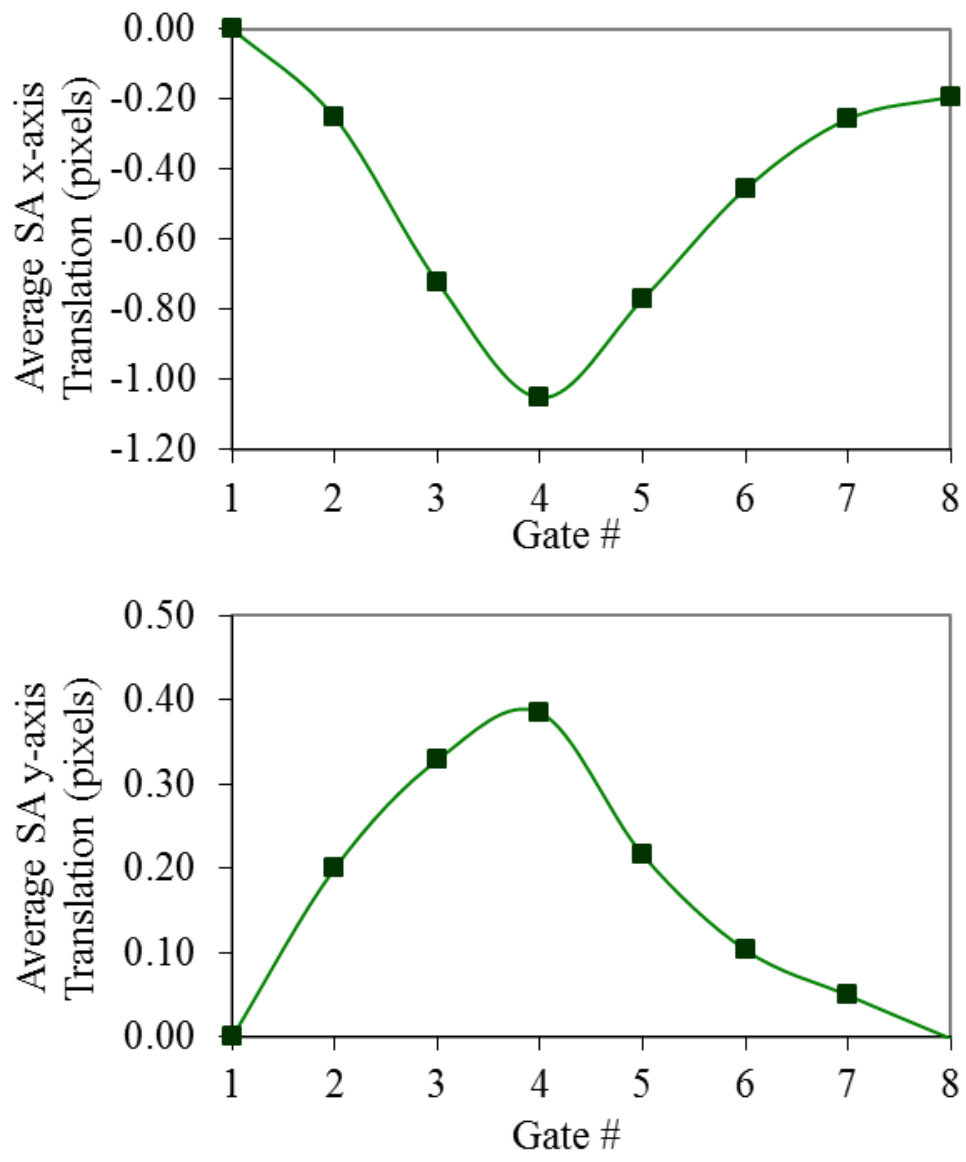

Figure 4.2: Average SA translation in the $x$-axis (top) and $y$-axis (bottom) as a function of gate number after applying translation correction

Applying translation correction had a significant impact on S, A_SD and A_E as shown in Table 4.3. With the exception of A_SD, all FA parameters decreased in MD in the septal wall while increasing in $\mathrm{MD}$ in the lateral wall. Boxplots comparing the differences between septal and lateral wall differences of 4 FA parameters are shown in 
Figure 4.3 while Figure 4.4 depicts the typical change in TACs due to translation correction applied in the septal and lateral wall.

Table 4.3: Impact of translation correction on FA parameters

\begin{tabular}{llll}
\hline & Without translation & With translation & p-value \\
\hline P_SD & $59 \pm 20$ & $56 \pm 19$ & 0.82 \\
P_BW & $230 \pm 79$ & $233 \pm 79$ & 0.71 \\
P_E & $0.78 \pm 0.05$ & $0.77 \pm 0.06$ & 0.17 \\
S & $0.81 \pm 0.13$ & $0.77 \pm 0.17$ & $\mathbf{0 . 0 0 3}$ \\
A_AVG & $0.41 \pm 0.07$ & $0.40 \pm 0.07$ & 0.37 \\
A_SD & $0.24 \pm 0.03$ & $0.20 \pm 0.02$ & $<\mathbf{0 . 0 0 1}$ \\
A_E & $0.73 \pm 0.02$ & $0.72 \pm 0.02$ & $<\mathbf{0 . 0 0 1}$ \\
\hline
\end{tabular}

A

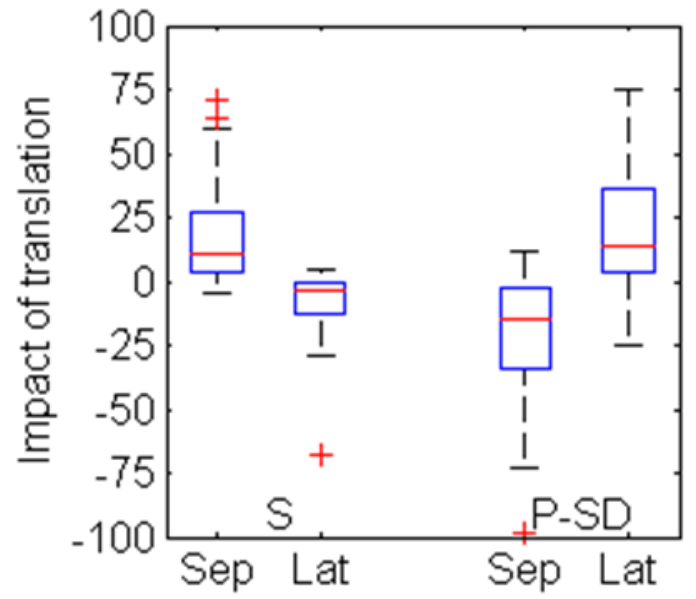

B

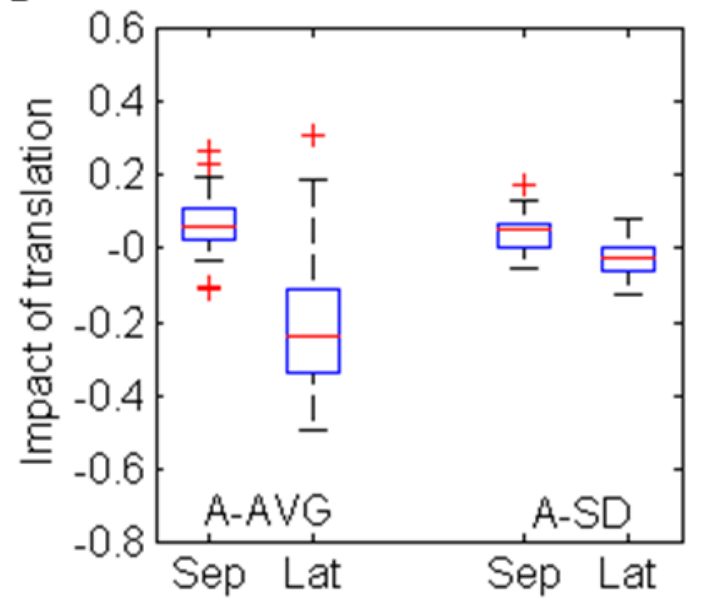

Figure 4.3: Change in septal and lateral wall a) S and P_SD and b) A_AVG and A_SD, after applying translation correction. S value was multiplied by 100 for display purposes. Sep $=$ septal, Lat $=$ lateral. The red line represents the median value while the top and bottom box lines are the $1^{\text {st }}$ and $3^{\text {rd }}$ quartiles. 


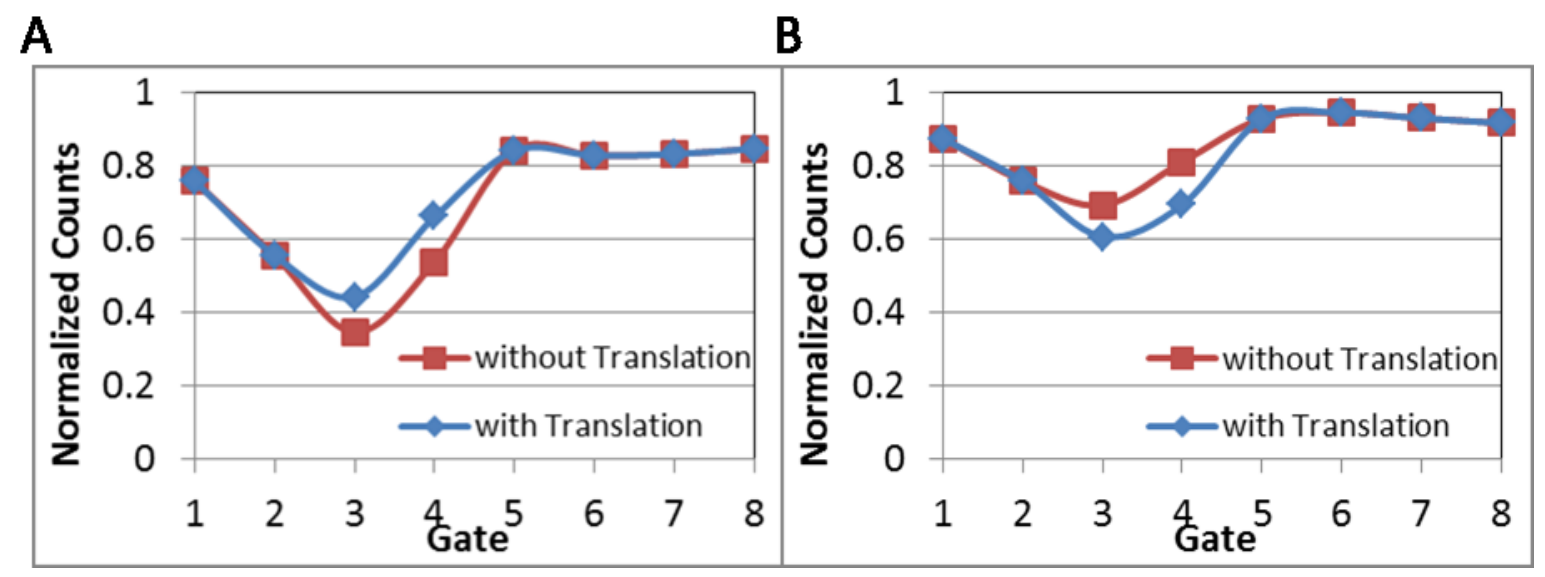

Figure 4.4: Typical change in TAC shape following translation correction in the a) septal wall and b) lateral wall.

\subsubsection{PREDICT CRT analysis}

All global FA parameters did not produce significant differences $(\mathrm{p}>0.05)$ between CRT responders and non-responders in the whole population, or in sub-populations of ischemia and non-ischemia. Similarly, in segmental analysis, no FA parameter was found to be significantly different $(\mathrm{p}>0.05)$ between CRT responders and non-responders in the apex, anterior, inferior and lateral walls. In the previous chapter, different lateral wall amplitude parameters analogous to scar size and score (AmpSize and AmpScore) were investigated and found to produce ROC AUCs of 0.68 but these were not significantly different than chance $(\mathrm{p}>0.05)$.

In septal wall FA, significant differences were obtained in the ischemic population between responder and non-responder groups in some FA parameters, as observed in 
Table 4.4. However, the significance was not preserved in the non-ischemic population and the whole population for any FA parameter.

Table 4.4: Septal wall FA parameters differences between responders and non-responders to CRT in the ischemic PREDICT population.

\begin{tabular}{llll}
\hline & Responder $(\mathrm{N}=17)$ & Non-responder $(\mathrm{N}=9)$ & p-value \\
\hline P_SD & $74 \pm 35$ & $58 \pm 37$ & 0.39 \\
P_BW & $242 \pm 105$ & $207 \pm 102$ & 0.55 \\
P_E & $0.69 \pm 0.03$ & $0.66 \pm 0.04$ & $\mathbf{0 . 0 5}$ \\
S & $0.64 \pm 0.24$ & $0.84 \pm 0.13$ & $\mathbf{0 . 0 2}$ \\
A_AVG & $0.19 \pm 0.06$ & $0.25 \pm 0.08$ & $\mathbf{0 . 0 5}$ \\
A_SD & $0.10 \pm 0.02$ & $0.14 \pm 0.05$ & $\mathbf{0 . 0 1}$ \\
A_E & $0.65 \pm 0.02$ & $0.65 \pm 0.02$ & 0.98 \\
\hline
\end{tabular}

\subsection{Impact of Translation Correction on prediction of CRT response}

Table 4.5 shows the change in p-values from Table 4.4 when applying translation correction to the patient data. Septal wall A_AVG and A_SD are no longer statistically significant ( $\mathrm{p}>0.05)$. 
Table 4.5: p-values of various septal wall FA parameters between CRT responders and nonresponders in the ischemic population

\begin{tabular}{lccccccc}
\hline & P_SD & P_BW & P_E & S & A_AVG & A_SD & A_E \\
\hline No Translation & 0.39 & 0.55 & $\mathbf{0 . 0 5}$ & $\mathbf{0 . 0 2}$ & $\mathbf{0 . 0 5}$ & $\mathbf{0 . 0 1}$ & 0.98 \\
With Translation & 0.21 & 0.42 & $\mathbf{0 . 0 2}$ & $\mathbf{0 . 0 2}$ & 0.18 & 0.07 & 0.72 \\
& & & & & & & \\
\hline
\end{tabular}

In the non-ischemic population, both septal P_E and septal A_AVG became statistically significant $(\mathrm{p} \leq 0.05)$ with the implementation of translation correction. No other global or segmental FA parameters achieved or lost statistical significance with the inclusion of translation correction in any sub-population.

Despite significantly changing the values of FA parameters, translation correction did not significantly affect the ability of the vast majority of FA parameters to predict response to CRT. Translation correction had a negative impact on two significant FA variables, septal A_AVG and septal A_SD, and thus was not implemented further.

The four septal wall FA parameters that provided significant differences between CRT responder and non-responder populations were further evaluated using ROC analysis. The results are given in Figure 4.5 and Table 4.6 below. Septal S produced the best ROC AUC of 0.78 and was the only parameter to be statistically significant compared to an AUC of 0.50 (chance line) $(\mathrm{p} \leq 0.05)$. However, none of the four FA parameters analyzed produced significantly different ROC AUC results compared to one another ( $p>0.05)$. 

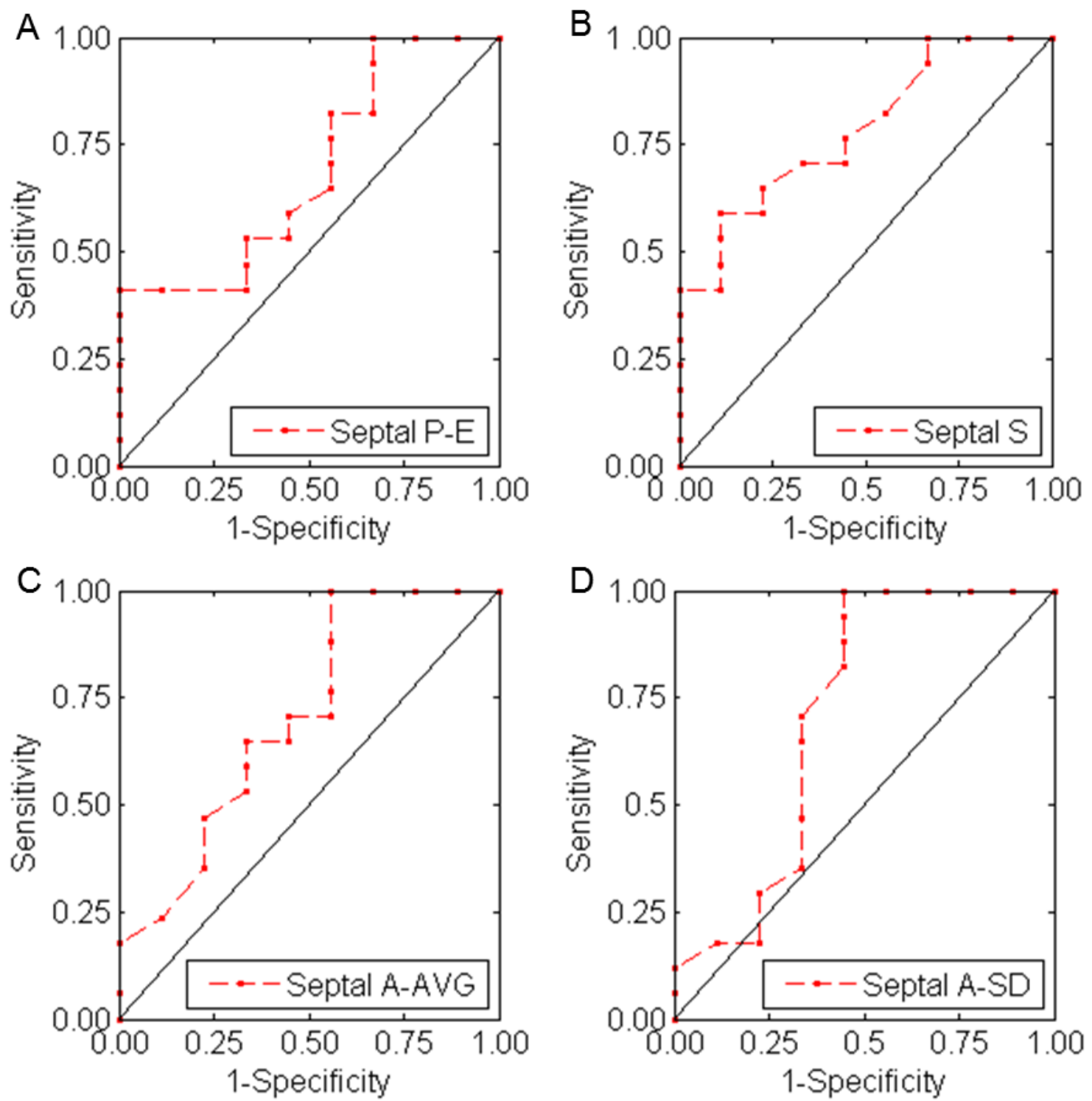

Figure 4.5: ROC curves for the PREDICT ischemic population for a) septal $P_{-} E$, b) Septal S, c) septal A_AVG and d) septal A_SD. 
Table 4.6: ROC AUC values for the PREDICT ischemic population using the best identified FA parameters

\begin{tabular}{lllll}
\hline & Septal P_E & Septal S & Septal A_AVG & Septal A_SD \\
\hline AUC & $0.69 \pm 0.11$ & $0.78 \pm 0.09^{*}$ & $0.71 \pm 0.10$ & $0.70 \pm 0.10$ \\
Sensitivity (\%) & 100 & 59 & 100 & 100 \\
Specificity (\%) & 33 & 89 & 44 & 56 \\
Cut-off & 0.63 & 0.75 & 0.30 & 0.13 \\
\hline AUC , area under the curve; ${ }^{*}$ ROC AUC values statistically significantly $(\mathrm{p} \leq 0.05)$ &
\end{tabular}

\subsubsection{RAFT CRT prediction}

Following the PREDICT analysis, 8 parameters were selected for further analysis with the RAFT population: global S, global P_E, global P_SD, septal S, septal A_AVG, septal A_SD, lateral A_AVG and lateral A_SD. Table 4.7 compares the means of the chosen FA parameters between CRT responder and non-responder groups in the ICD and CRT RAFT population. 
Table 4.7: Comparison of pre-selected FA parameters means between responders and nonresponders to CRT in the RAFT ICD and CRT population.

\begin{tabular}{|c|c|c|c|}
\hline \multicolumn{4}{|c|}{ Non-Response criteria: CHF Hospitalization } \\
\hline & Responder $(\mathrm{N}=85)$ & Non-responder $(\mathrm{N}=26)$ & p-value \\
\hline Global P_SD & $52 \pm 16$ & $43 \pm 17$ & 0.01 \\
\hline Global P_E & $0.75 \pm 0.05$ & $0.77 \pm 0.05$ & 0.09 \\
\hline Global S & $0.83 \pm 0.12$ & $0.88 \pm 0.09$ & 0.05 \\
\hline Septal S & $0.70 \pm 0.24$ & $0.76 \pm 0.22$ & 0.16 \\
\hline Septal A_AVG & $0.21 \pm 0.08$ & $0.21 \pm 0.07$ & 0.47 \\
\hline Septal A_SD & $0.11 \pm 0.05$ & $0.11 \pm 0.04$ & 0.88 \\
\hline Lateral A_AVG & $0.53 \pm 0.12$ & $0.54 \pm 0.10$ & 0.87 \\
\hline Lateral A_SD & $0.18 \pm 0.03$ & $0.17 \pm 0.03$ & 0.37 \\
\hline \multicolumn{4}{|c|}{ Non-Response criteria: Death } \\
\hline & Responder $(\mathrm{N}=85)$ & Non-responder $(\mathrm{N}=26)$ & $\mathrm{p}$-value \\
\hline Global P_SD & $50 \pm 15$ & $48 \pm 21$ & 0.44 \\
\hline Global P_E & $0.76 \pm 0.05$ & $0.76 \pm 0.06$ & 0.93 \\
\hline Global S & $0.84 \pm 0.11$ & $0.84 \pm 0.12$ & 0.66 \\
\hline Septal S & $0.71 \pm 0.23$ & $0.73 \pm 0.25$ & 0.44 \\
\hline Septal A_AVG & $0.20 \pm 0.07$ & $0.23 \pm 0.09$ & 0.08 \\
\hline Septal A_SD & $0.11 \pm 0.04$ & $0.13 \pm 0.05$ & 0.01 \\
\hline Lateral A_AVG & $0.54 \pm 0.11$ & $0.50 \pm 0.13$ & 0.17 \\
\hline Lateral A_SD & $0.18 \pm 0.03$ & $0.18 \pm 0.03$ & 0.89 \\
\hline
\end{tabular}

Non-Response criteria: Death or CHF hospitalization 


\begin{tabular}{llll}
\hline & Responder $(\mathrm{N}=69)$ & Non-responder $(\mathrm{N}=42)$ & p-value \\
\hline Global P_SD & $52 \pm 15$ & $46 \pm 19$ & $\mathbf{0 . 0 5}$ \\
Global P_E & $0.77 \pm 0.05$ & $0.75 \pm 0.05$ & 0.14 \\
Global S & $0.83 \pm 0.11$ & $0.86 \pm 0.11$ & 0.09 \\
Septal S & $0.69 \pm 0.24$ & $0.74 \pm 0.22$ & 0.24 \\
Septal A_AVG & $0.20 \pm 0.07$ & $0.22 \pm 0.09$ & 0.13 \\
Septal A_SD & $0.11 \pm 0.04$ & $0.12 \pm 0.04$ & 0.08 \\
Lateral A_AVG & $0.54 \pm 0.12$ & $0.52 \pm 0.11$ & 0.22 \\
Lateral A_SD & $0.18 \pm 0.03$ & $0.17 \pm 0.03$ & 0.33 \\
\hline
\end{tabular}

Results varied considerably by response criteria. In the CHF hospitalization outcome analysis, global parameters ( $\mathrm{P} \_\mathrm{SD}$ and $\left.\mathrm{S}\right)$ were the only parameters significantly different between responders and non-responders ( $\mathrm{p}=0.01$ and 0.05 respectively). Using death as an outcome analysis, septal A_SD was the only significant parameter $(p=0.05)$ (the $p$ value for septal A_AVG was 0.08). In the combination death or CHF hospitalization outcome analysis, global P_SD was the only statistically significant parameter $(p=0.05)$. The three FA parameters that provided significant differences between populations and septal A_AVG were further evaluated by ROC analysis. These results are shown in Table 4.9 and Figure 4.6.

Each parameter was also analyzed using a Cox proportional hazard model. The analysis was repeated by adjusting for each variable individually in the model as well as for each 
of the three outcomes but no variable produced significant results. A further interaction test revealed that septal A_AVG and to a lesser extent septal A_SD (Table 4.8), produced significantly different results between the ICD group and the ICD and CRT group for death.

Table 4.8: Comparison of responder septal A_AVG and septal A_SD values in the ICD and ICD+CRT RAFT population using death as non-response criteria.

\begin{tabular}{llll}
\hline & ICD $(\mathrm{N}=103)$ & ICD/CRT $(\mathrm{N}=111)$ & p-value \\
& Median $(\mathrm{Q} 1, \mathrm{Q} 5)$ & Median $(\mathrm{Q} 1, \mathrm{Q} 5)$ & \\
\hline Septal A_AVG & $0.20(0.15,0.25)$ & $0.19(0.16,0.25)$ & 0.05 \\
Septal A_SD & $0.11(0.08,0.14)$ & $0.10(0.08,0.13)$ & 0.08 \\
\hline
\end{tabular}



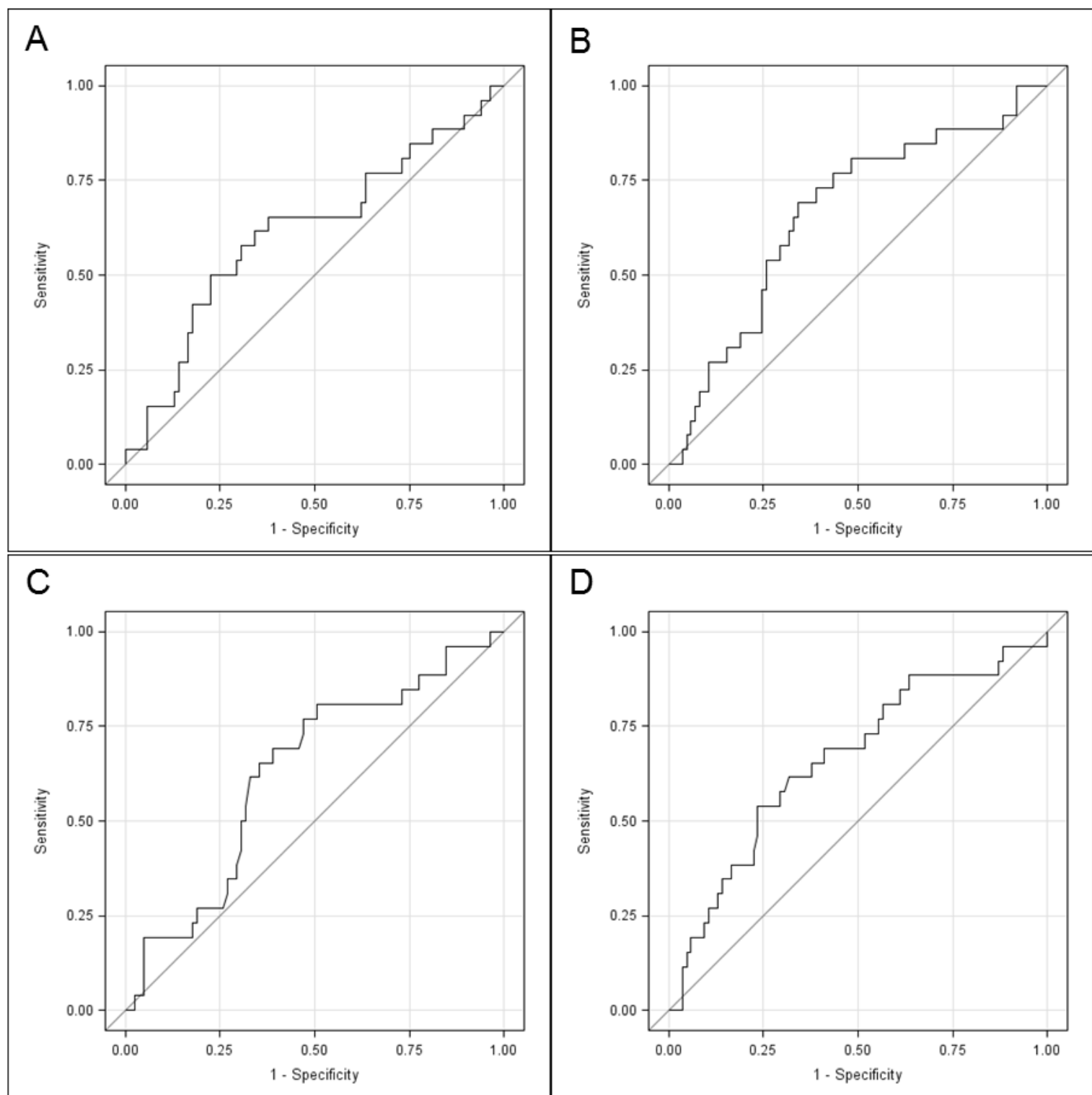

$\mathrm{D}$

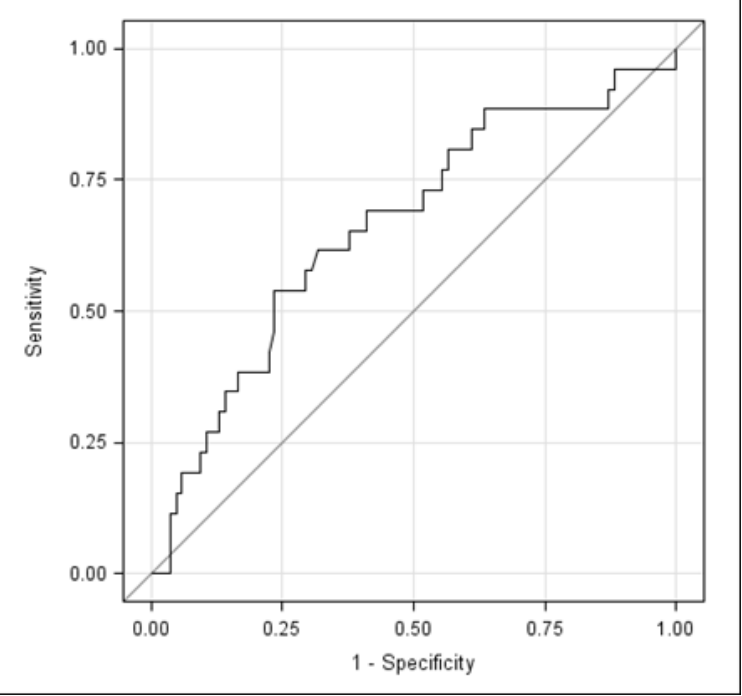

Figure 4.6: ROC curves for the RAFT ICD+CRT population for a) septal A_AVG, b) Septal A_SD using death as non-response criteria and, c) global S and d) global P_SD using CHF hospitalization as non-response criteria. 
Table 4.9: ROC AUC values for the RAFT ICD+CRT population using the best identified FA parameters

\begin{tabular}{lllll}
\hline & Septal A_AVG & Septal A_SD & Global S & Global P_SD \\
\hline ROC AUC & $0.61 \pm 0.07$ & $0.66 \pm 0.06^{*}$ & $0.63 \pm 0.06^{*}$ & $0.66 \pm 0.06^{*}$ \\
Sensitivity (\%) & 65 & 69 & 69 & 54 \\
Specificity (\%) & 62 & 66 & 61 & 76 \\
Cut-off & 0.20 & 0.11 & 0.88 & 39 \\
\hline AUC , area under the curve; ${ }^{*}$ ROC AUC values statistically significantly $(\mathrm{p} \leq 0.05)$ &
\end{tabular}

Septal A_SD, global S and global P_SD yielded ROC AUC values significantly better than 0.50 (chance line).

\subsection{Discussion}

In the RAFT population, class II CHF patients presented an improved chance of survival over class III CHF patients. This is not a surprise as patients with worst CHF may already be too damaged to be resynchronized. Furthermore, if reverse remodeling does not occur, then death is more likely in class III since symptoms are more progressed than in class II. It may be useful to pursue analysis stratifying further by NYHA class to determine the rates of non-response vary between these populations.

Gating correction was implemented in 17 patients from the RAFT study, all of which underwent SPECT RNA scans ECG-gated to 16 frames. Thresholds of $60 \%$ of maximum activity for dropping a gate and $90 \%$ for rescaling were used in the study. Various cutoff values were not investigated to determine optimal cut-offs though the values chosen 
appeared to adequately restore TACs to realistic shapes. Table 4.2 showed that P_SD, P_E and S were significantly affected by gating correction. Furthermore, phase based parameters tended to overestimate MD without gating correction while amplitude-based underestimated MD. For amplitude, this may be explained by the fact that low counts in the final gates fell below the end-systolic counts causing a greater spread of counts between the maximum and minimum, artificially increasing A_AVG (reducing MD). In the case of phase overestimating MD, it is possible that without correction, the minima of the sinusoidal fit was placed incorrectly near the last gates due to low counts while in other cases when the low gate counts did not dip below the end-systolic time frame (typically near a middle gate), then the minima of the fitted curve correctly passed through end-systole. When both scenarios occurred, a greater dispersion of $\mathrm{P} \_$AVG was observed and thus an increase in P_SD occurred. Interestingly, Ludwig et al. (141) reported that gating artifacts caused P_SD to be underestimated, which is in direct contrast to results obtained in this study. It is unclear why the results are contradictory, but the two studies differed considerably. In the Ludwig et al. study (141), different threshold values were used for rescaling and gates were never dropped. Furthermore, gating atifacts were induced by LV pacing and FA was performed on SPECT MPI scans, measuring change in myocardial wall thickening rather than blood pool activity.

Whereas gating artifacts are false variations in the counts that must be corrected, translational motion is an intrinsic part of the heart's motion and it was unclear whether or not it is beneficial to correct for it. Results showed that on average the LV moved in towards the septum by about $3.5 \mathrm{~mm}$. To a lesser extent, the LV also moved anteriorly, 
on average about $1.8 \mathrm{~mm}$ during contraction. It is important to note that translation correction was not implemented on a sub-pixel level, but rather the ROIs were shifted by integer values, where necessary, at each gate. This may have reduced the accuracy of the translated TAC shape. The maximum impact of translation occurred near the endsystolic gates (Figure 4.2) and was on the order of a single pixel shift. Thus, gates outside of end-systole may not have been translated due to rounding. This is evidenced in Figure 4.4 which shows the impact of applying the translation correction on a typical TAC, where only gates 3 and 4 were translated. It can be qualitatively observed from Figure 4.4 that amplitude values obtained from FA will be affected with the current translation correction algorithm and that, the true shape of the TAC may not be accurately depicted. In a different methodology, such as cluster analysis (chapter 5), where the full shape of the TAC is analyzed, a discrete translation correction may cause a loss of information. This is not believed to be as large an issue for FA because the fitting process forces a sinusoidal fit through the data. Without implementation of a translation correction algorithm, the ROIs remained fixed during contraction even if translation occurred. In a heart that shifted towards the septum, an increase in counts acquired at end-systole was observed as the ROIs placed around the septal wall were now located inside the LV. Therefore, smaller FA amplitudes values were observed in septal TACs. By implementing a translation correction, the difference between the maximum and minimum increased as observed in Figure 4.4. As the LV moved towards the septum, the ROIs positioned on the lateral wall recorded less counts as the heart contracted, as the ROIs moved outside the LV, causing the opposite effect observed in septal TAC. 
Correcting for translational motion had a significant impact on several FA parameters, notably S, A_SD and A_E $(\mathrm{p}<0.01)$. Amplitude based FA parameters presented significant differences because translation correction affected the counts acquired, mostly near end-systole, but the relative timing of the contraction (phase) likely did not change. This can be qualitatively observed in Figure 4.4. In segmental analysis septal FA parameters and lateral FA parameters behaved differently once the translation correction was applied (see Figure 4.3) due to the differences outlined previously.

Prior to evaluating the impact of translation correction on predicting CRT response, the original methodology was evaluated. Using the PREDICT population, a total of 42 FA parameters were tested to determine the best at predicting response to CRT. Similar to the results from chapter 3, no FA parameters were found to be predictive of response in the whole population. As shown in Table 4.4, significant differences occurred only with septal FA in the ischemic population. Of the 7 septal FA parameters, P_E, S, A_AVG and A_SD presented significant differences between CRT responder and non-responders groups $(\mathrm{p} \leq 0.05)$. Of note, 3 of the 4 parameters included amplitude in the calculation, which suggests that amplitude is a useful MD parameter.

The same analysis was then performed with the inclusion of the translation correction algorithm. In the vast majority of cases, no changes in statistical significance occurred for any FA parameter. In the ischemic population, two parameters, septal A_AVG and septal A_SD, were negatively impacted, losing significance. However, septal A_AVG became a significant predictor of response in the non-ischemic population. No other 
significant changes were observed in any population. These results indicate that correcting for translation LV wall motion has little impact on FA. However, the fact that A_AVG and A_SD were no longer significant predictors suggests that the extent of translation of the LV may be providing useful information and that it should be not corrected for. Thus, translation correction was not implemented further.

During contraction, it is also possible for the heart to rotate and this would also affect the counts recorded in a fixed ROI. No rotation correction algorithm was applied to the data in this study. Using SPECT RNA FA techniques, wall motion was assessed through the change in counts recorded in the ventricle as the heart contracts and there is no quantifiable means of directly measuring rotation and its impact on the ROI counts. Based on the minor impact translation correction had on FA, it is not expected that a rotation correction algorithm would impact FA significantly but it was not possible to verify this with the data.

The four parameters that were significant predictors of response to CRT were evaluated using ROC analysis. While septal S was the only parameter to produce a statistically significant ROC AUC value, it was not statistically superior to the other parameters which all showed similar ROC AUC results around 0.70 . Based on these results, septal S, septal A_AVG and septal A_SD were selected for analysis in the RAFT population. As discussed previously, the lateral wall has been cited as being the critical LV segment for CRT response, notably in scar studies (see section 2.3), and thus, lateral wall A_AVG and lateral A_SD were included for analysis with the RAFT population. Finally, global 
P_SD, global P_E and global S were also included in this study as they are commonly found FA parameters in the literature, making them a good comparison point.

In the RAFT population, three different non-response criteria were considered: death, CHF hospitalization and combination death and/or CHF hospitalization. Results varied between the three outcomes criteria (Table 4.7). In the CHF hospitalization group, significant differences existed between CRT responders and non-responders for global P_SD and global S only. This was not expected as global FA parameters had not shown any predictive ability previously. In the death outcome analysis, only septal A_SD was significant $(\mathrm{p}=0.01)$ between groups while in the combination outcome group, global P_SD was the only FA parameter to retain significance $(\mathrm{p}=0.05)$. Septal $\mathrm{S}$ was no longer significant in any outcome group despite producing the best results in PREDICT population. ROC analysis was performed on the four parameters that indicated the greatest ability to predict response to CRT. The ROC AUC values tended to be smaller (0.61 to 0.66 ) than those obtained in the PREDICT ischemic population 0.70 to 0.78 ), but the differences were not significant $(\mathrm{p}>0.05)$. However, septal A_SD, global S and global P_SD all produced results significantly better than chance $(\mathrm{p} \leq 0.05)$. Optimal sensitivity and specificity were $69 \%$ and $66 \%$ respectively for septal A_SD and 54\%/ 76\% for global P_SD.

It is not clear why global FA parameters fared better at predicting CHF hospitalization while septal FA parameters were significant predictors of death. It is also of note that both FA parameters that predicted CHF hospitalization included the phase component 
while only amplitude factored in predicting death. These results suggest that a patient with CHF needed a certain degree of global phase dispersion before CRT is beneficial, perhaps so that there were enough dyssynchronous regions to be resynchronized by the pacemaker. Otherwise, these patients did not improve and eventually returned to the hospital with similar or worsening symptoms. Amplitude, or extent of contraction, in the septal wall was key to survival, suggesting that if too many regions of the septal wall were poorly contracting, it was too late, and death is likely. Results have shown that translation of the LV affects the TAC, especially in the septal and lateral wall and it was previously discussed that translational wall motion reduced the significance of septal A_AVG and septal A_SD in the PREDICT population. This may indicate that magnitude of translation played a part in non-response (notably death) and that septal amplitude parameters were picking up on this effect since translational motion was not corrected for.

Of note, the FA parameters that achieved significance in the PREDICT population matched up better with RAFT results using death as the outcome rather than CHF hospitalization. The PREDICT response outcome was based on LV reverse remodeling which suggests that a lack of remodeling following CRT is an indicator of death and could be used to intervene in CRT recipients before it is too late.

Further analysis was undertaken to compare the ICD and ICD+CRT populations. A Cox proportional hazards model indicated that septal A_AVG showed significant interaction with CRT for predicting death. Those that survived with a CRT had lower septal 
A_AVG values on average, indicating that CRT offers a clinical benefit to patients with high MD. In other words, using death as the outcome, the ICD+CRT group had increased survival odds at higher MD values, as assessed by septal A_AVG, than in the ICD group.

A limitation of this study was that segmental analysis was performed on a 5 segment model. Implementing a 9 or standard 17 segment LV model may improve the localization of MD and thus help improve the ability of FA parameters at predicting CRT response. The impact of smaller LV regions to improve the ability of CRT response warrants further investigation.

Although it has been reported in other studies as providing an increased discrimination between response and non-response, the potential of combining two or more FA parameters to provide additional information was not considered in this study and remains a possible future investigation.

\subsection{Conclusion}

New and previously defined global and segmental FA parameters were analyzed to determine the best FA parameters to predict response to CRT. Septal amplitude-based parameters (A_AVG, A_SD) produced the best results for predicting death while global P_SD and $\mathrm{S}$ were the best for predicting CHF hospitalization. ROC analysis of these variables produced modest but significant results of sensitivity and specificity of $69 \%$ and $66 \%$ for septal A_SD and 54\% and 76\% for global P_SD respectively. A translational 
wall motion correction algorithm was tested but was not considered beneficial as the ability of FA parameters to predict response to CRT did not improve in the majority of cases, while losing significance in septal A_AVG and A_SD. 


\section{Chapter 5}

\section{Development of cluster analysis}

\subsection{Introduction}

Results from the previous chapter suggested that despite producing good results for predicting CRT response, FA may be limited in its potential at predicting CRT outcome. This may be due to the fact that by extracting only one or at most two parameters from a first order Fourier fit, FA is losing valuable information from the TAC.

A new wall motion analysis technique may be needed to provide additional information relating to mechanical dyssynchrony that is not supplied by FA. Other SPECT studies have identified this disadvantage and have attempted different approaches to FA for assessing dyssynchrony (142-144). In this chapter, we propose adapting an existing technique, cluster analysis, to directly evaluate the TAC with the aim of improving the assessment of quantification of mechanical dyssynchrony.

Cluster analysis is a data analysis technique which classifies objects into groups, or clusters, based on the comparison criteria. Jain et al. (145) and Du et al. (146) provide good overviews of different clustering methods, such as k-means and fuzzy clustering 
algorithms. One application of cluster analysis is pattern recognition which has led to applications in medical imaging. Several PET studies (147-149) have used cluster analysis to improve the signal-to-noise ratio (147). In dynamic PET imaging, for each voxel in an image, an uptake TAC is created and assigned to a cluster group based on its tissue kinetics. The PET image can then be segmented by creating ROIs based on cluster groups and physical proximity of the voxels, enabling automatic acquisition of physiological parameters. Similarly in functional MRI analysis, cluster analysis has been used to identify and group different tissue activation levels in order to segment the data based on their activation levels (150-152).

In this chapter, the cluster analysis method is adapted to quantify mechanical dyssynchrony from cardiac TACs. Cluster algorithms were developed and optimized to differentiate normal (synchronous) TACs from dyssynchronous TACs, which may lead to a better understanding of dyssynchrony patterns. Cluster analysis was then used to determine its ability to predict CRT response using cluster size and score metrics. Results were compared to SPECT RNA FA and PET scar analysis methods.

\subsection{Materials and Methods}

\subsubsection{Patients}

Evaluation of the clustering approach to TAC classification was done retrospectively. A database of normal cardiac subjects (see section 2.5.1) was used to define normal wall motion TACs and then the clustering method was tested on an abnormal group of heart failure patients (the PREDICT population) (1) (see section 2.5.2). 
As discussed previously, the PREDICT population consisted of 49 patients who qualified for pacemaker implantation based on CRT selection criteria (LVEF $<30 \%$, QRS $>120$ ms, New York Heart Association (NYHA) class II or III) and who received both a planar and SPECT RNA scan, as well as PET perfusion and viability scans prior to the CRT surgery.

Of the 49 patients, $27(60 \%)$ were diagnosed with ischemic cardiomyopathy defined as having a history of myocardial infarction and severe stenosis $(\geq 70 \%$ in at least 2 major arteries). The remaining $22(40 \%)$ patients presented with no history of infarction or coronary disease and so were diagnosed with non-ischemic cardiomyopathy $(1,126)$.

\subsubsection{SPECT RNA Data Acquisition}

The imaging acquisition protocol used for the PREDICT study and the normal cardiac database follow the standard clinical UOHI protocol and is outlined in section 3.2.2.2.

In-house software developed for SPECT RNA FA (see section 2.2.2) was adapted to assess each patient with both FA and cluster analysis. In brief, a semi-automated algorithm segmented the myocardial LV wall into 568 ROIs based on 18 linearly interpolated SA slices from LV apex to base. The locations of the ROIs were fixed and 568 TACs were produced from the total counts in each of the ROIs over the 8 gates of the SPECT RNA image series. From there, the algorithms for FA (see section 2.2.1) and cluster analysis (outlined below) differed. 


\subsubsection{Cluster analysis}

\subsubsection{TAC normalization}

Each TAC was normalized to account for variability in absolute count levels caused by differences in total attenuation or activity administered. Three different normalization techniques were compared in 50 normal subjects to select the method which minimized the inter- and intra-subject variability. The three techniques were global, segmental, and individual normalization.

Global normalization used all 568 TACs of a patient to calculate a single average count value. Each data point on each of the 568 TACs was then divided by this single average count value to produce a normalized value. This approach reduced inter-subject variability but maintained intra-subject variation.

Segmental normalization divided the heart into 5 segments: inferior, anterior, septal, lateral and apical (Figure 2.5). Five different average count values were created by taking the averages of all TACs located in each separate segment. A TAC located in a particular segment was then normalized to the average count value of its respective segment. This approach reduced overall segmental variations but maintained local variability. 
For individual normalization, each TAC was normalized to its own count average. This approach minimized intra-subject TAC variation. A sample individual normalization is shown in Figure 5.1.
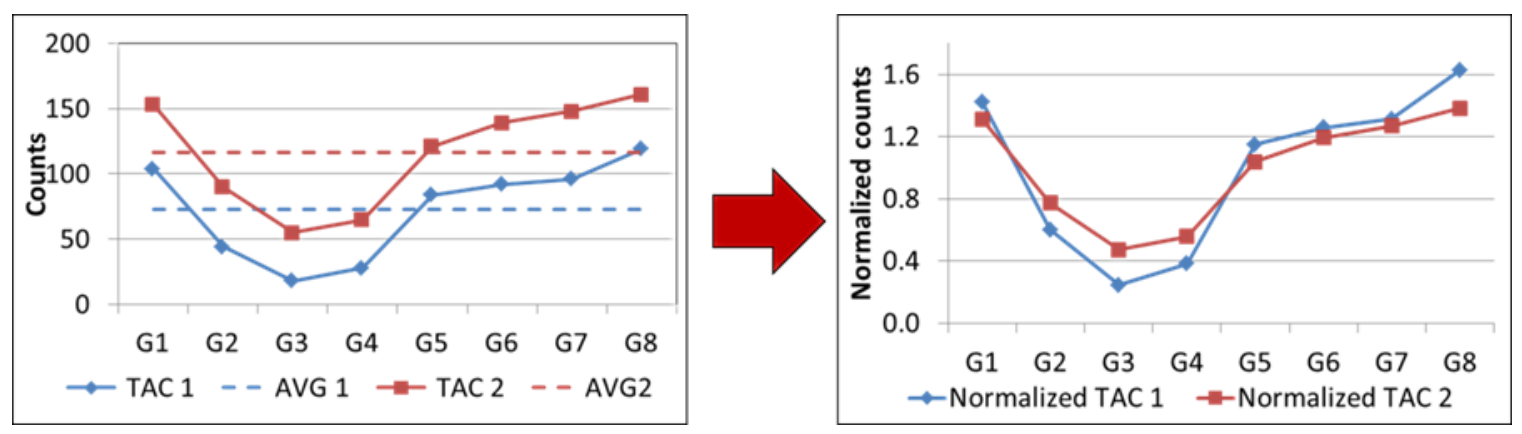

Figure 5.1: Two sample unnormalized TACs (left) are each normalized to their own average (dashed line) to produce two individually normalized curves (right). G1 represents gate 1, G2 = gate 2 , etc.

\subsubsection{Development of a normal subject database}

A database of 50 normal cardiac subjects was analyzed to establish global and segmental reference TACs. For the global reference TAC, the 568 normalized TACs of each individual subject were averaged at each of the 8 time frames to create a single global TAC. The global TACs from the 50 subjects were averaged to create a single mean global TAC (and standard deviation) representing a normal LV cardiac wall motion TAC.

For the 5 segmental reference TACs, all the normalized TACs within a segment of an individual subject were averaged to create that subject's 5 mean segmental TACs. This was repeated for each normal subject. A single mean (and standard deviation) TAC was created for each of the 5 segments by averaging the mean TACs of the 50 normal subjects within a segment. 


\subsubsection{K-means}

For cluster analysis, the 568 TACs were individually normalized and exported to a data file for further analysis using MATLAB software. The patient data were analyzed using two different automated cluster techniques: K-means and normal average.

The K-means approach proceeded as a two-step algorithm. In the first step, the algorithm randomly selected 2 observations as starting points for 2 different clusters and began the first iteration. At each iteration, every observation was placed in the cluster that is closest to it. At the end of the iteration, the mean of each cluster was recalculated. This new mean then served as the starting point for the next iteration's clusters, where all observations were reassigned. This continued for 100 iterations (default setting) or until the mean of the clusters remained unchanged, whichever occurred first. The mean clusters of the final iteration served as starting points for the second step of the algorithm, where there was a single pass through all observations. Each observation, one-by-one, was assigned to the closest cluster and the mean of a cluster was immediately recalculated once an observation was assigned.

For this analysis, a single TAC represented an observation, and each observation was assigned to the cluster which minimized the Euclidian distance between the cluster mean and the observation. For each patient, the size of the cluster with the smallest number of members was used as a measure of LV dyssynchrony. 


\subsubsection{Normal Average}

Unlike the K-means algorithm, the normal average algorithm did not use its own patient data as a basis for its clusters, but rather used a pre-defined mean TAC obtained from the database of subjects with normal cardiac function. A mean normal global TAC and 5 segmental TACs were defined using the normal population (section 5.2.3.2). In segmental analysis, for example, a septal TAC was compared to the mean normal septal TAC only. Briefly, if a TAC was sufficiently different from the pre-defined 'normal' TAC, it was placed in an 'abnormal' or dyssynchronous cluster; otherwise it was placed in the 'normal' cluster. To facilitate comparison, each data point on a TAC was directly compared to the equivalent data point on the reference normal TAC. If a data point was outside an acceptable range of the reference point, it was considered an outlier. Once sufficient data points on a single TAC were deemed outliers, the TAC was placed in the appropriate abnormal cluster; otherwise it was placed in the normal cluster.

For normal average cluster analysis, several input metrics were varied in order to determine optimal settings:

1. Standard deviation $(\sigma)$ was used to determine if a data point was within the acceptable range of the reference mean normal data point. A threshold of $1 \sigma, 2 \sigma$ and $3 \sigma$ were considered for the analysis.

2. The number of clusters was chosen to be either 2 or 5 . In the 5-cluster analysis, clusters were defined as in Table 5.1 below and a score of $0-4$ was assigned to each observation based on the degree of abnormality as described in the third row of Table 5.1. 
Table 5.1: Corresponding score per number of outlier data points in a TAC for each cluster group

\begin{tabular}{llllll}
\hline \# Outliers & 0 & 1 & 2 & 3 & 4 or more \\
\hline Assigned score & 0 & 1 & 2 & 3 & 4 \\
\hline
\end{tabular}

3. For the 2-cluster group, the threshold number of outlier data points required before a TAC was assigned to the abnormal cluster was varied from 1 to 3 .

In order to determine optimal settings, all combinations of metrics were investigated. The percent of all observations classified in the abnormal cluster was used as a measure of LV dyssynchrony for 2 cluster analysis. For 5-cluster analysis, the total score, expressed as a percentage of total possible score, was used as a marker of dyssynchrony.

\subsubsection{CRT response}

Patients were re-evaluated 3 months after CRT surgery for functional improvement. Patients were characterized as responders if ejection fraction improved by at least $5 \%$ or end-systolic volume was reduced by at least $10 \%$ with respect to their baseline scan. The protocol has been previously described in section 3.2.3.

\subsubsection{Data Analysis}

Inter-subject variability curves were calculated by determining the standard deviation of the 50 mean normal TACs at each data point. To determine intra-subject variability, the 
standard deviation of all 568 TACs of a single patient was first calculated at each data point. Then, the average of all standard deviation values across all patients was found for each data point. Both, inter- and intra-subject variability curves were then expressed as a percentage of the mean reference curve value. This process was performed for all three normalization techniques to determine the method that produced the least variability.

Cluster analysis results were then compared to the best results obtained from PET scar size analysis results (chapter 3) and SPECT RNA FA results (chapter 4).

ROC curves were created for each combination of cluster analysis metrics investigated. ROC AUC values were calculated for each parameter combination investigated. A Cohen's kappa value ( $\kappa)$ was calculated as a measure of concordance with the results of the follow-up RNA exam and was used to determine optimal operating point on an ROC curve and to compare optimal sensitivity and specificity combinations between curves. A Wilcoxon rank sum test was used to determine if significant differences existed between population means while a Fisher exact test was used for comparison of categorical variables. A p-value of 0.05 or less was considered statistically significant for all tests.

\subsection{Results}

\subsubsection{Patients}

A total of 48 patients were successfully analyzed using cluster analysis, FA and scar size analysis. One patient was excluded due to poor SPECT RNA image quality. 30 (63\%) patients were identified as responders while 18 (37\%) were non-responders. Patient 
demographics for all 48 patients, stratified by responder status, are given in Table 5.2 alongside demographics for the normal subject database.

Table 5.2: Patient demographics for the normal population, CRT responder and non-responder groups.

\begin{tabular}{llll}
\hline & Normals & Responders & Non-Responders \\
& $(\mathrm{N}=50)$ & $(\mathrm{N}=30)$ & $(\mathrm{N}=18)$ \\
\hline Age & $57 \pm 16$ & $68 \pm 9$ & $67 \pm 10$ \\
LVEF (\%) & $61 \pm 7 \% *$ & $21 \pm 7$ & $23 \pm 8$ \\
LVESV (mL) & $41 \pm 11^{*}$ & $232 \pm 77$ & $231 \pm 136$ \\
QRS duration (ms) & $88 \pm 10^{*}$ & $172 \pm 25$ & $162 \pm 27$ \\
Gender (M/F) & $31 / 19$ & $23 / 7$ & $17 / 1$ \\
NYHA Class (II/III) & $\mathrm{n} / \mathrm{a}$ & $10 / 21$ & $3 / 15$
\end{tabular}

$\overline{\mathrm{LVEF}}=$ left ventricular ejection fraction; LVESV = left ventricular end-systolic volume; NYHA $=$ New York Heart Association; ${ }^{*} \mathrm{p} \leq 0.05$ between normal and both responder and non-responder groups. $\mathrm{p}>0.05$ for all variables between responder and non-responder groups

\subsubsection{SPECT RNA Cluster Analysis}

Cluster analysis results using both the normal average and K-means algorithm were analyzed for the entire 48 patient population as well as separately for ischemic and nonischemic cardiomyopathy subgroups. All combinations were evaluated globally as well as in all 5 polar map segments. 


\subsubsection{TAC normalization}

The inter- and intra-subject variability curves for each normalization technique are presented in Figure 5.2 and summarized in Table 5.3. The values in Table 5.3 represent the standard deviations averaged over the 8 time frames. All three normalization techniques provided similar inter-subject variability but individual normalization produced the least intra-patient variability, averaging $15 \%$.

Table 5.3: Intra- and inter- subject variability of the 3 different normalization techniques, averaged over the 8 time frames.

\begin{tabular}{lccc}
\hline & Global & Segmental & Individual \\
\hline Intra-subject variation & $34 \%$ & $25 \%$ & $15 \%$ \\
Inter-subject variation & $11 \%$ & $12 \%$ & $12 \%$ \\
\hline
\end{tabular}

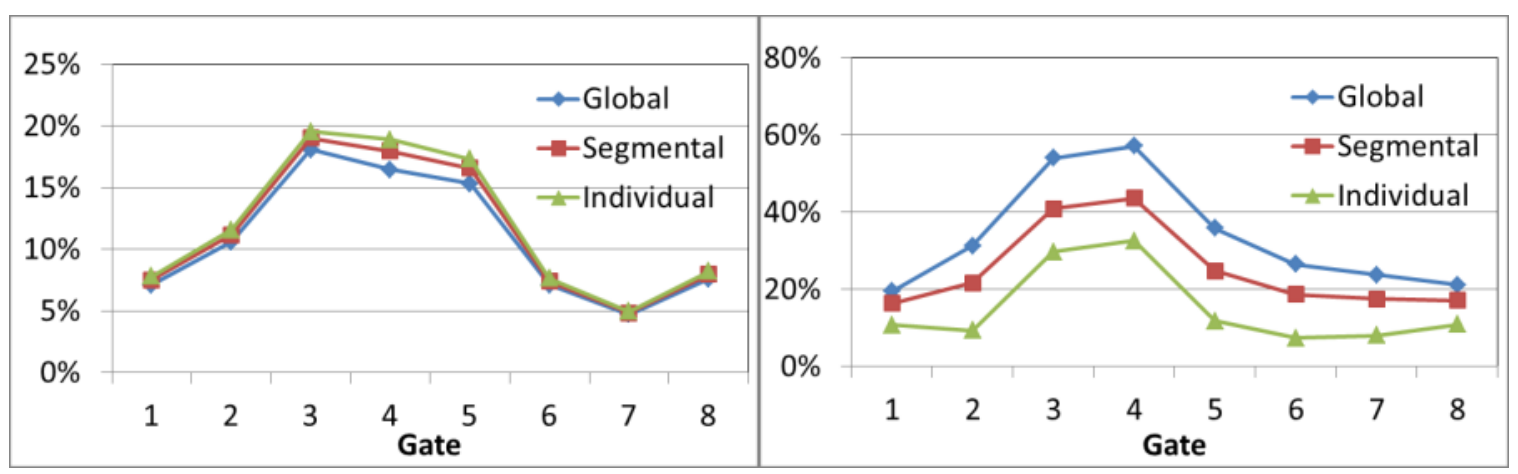

Figure 5.2: Inter- and intra-subject variability at each gate for the 3 different normalization techniques. 


\subsubsection{K-means}

Table 5.4 summarizes ROC AUC results of cluster analysis using the K-means algorithm. The largest ROC AUC s $(\sim 0.70)$ occurred in the septal wall. Near identical values of septal wall ROC AUC s were reported in the different sub-populations.

Table 5.4: Global and segmental ROC AUC values for all populations using the K-means algorithm

\begin{tabular}{llll}
\hline & Global & Septal & Lateral \\
\hline All & $0.51 \pm 0.09$ & $0.70 \pm 0.08$ & $0.61 \pm 0.09$ \\
Ischemic & $0.55 \pm 0.12$ & $0.71 \pm 0.10$ & $0.68 \pm 0.12$ \\
Non-ischemic & $0.61 \pm 0.13$ & $0.69 \pm 0.11$ & $0.50 \pm 0.13$ \\
\hline
\end{tabular}

\subsubsection{Normal Average}

The best ROC AUC results were found using the septal wall for all population subgroups. The whole population and the ischemic sub-group achieved their largest AUCs using the $\mu \pm 2 \sigma$ outlier threshold while the non-ischemic population produced best results using the $\mu \pm 3 \sigma$ outlier threshold. The best results for each population are presented in Table 5.5. An optimal sensitivity of $94 \%$ and $67 \%$ specificity was obtained for the "3 or more outliers before abnormal observation" at a cutoff of $52 \%$ of the TACs classified as abnormal in the ischemic population. 
Table 5.5: Best cluster analysis ROC AUC results for all populations. Best results for each population were obtained in the septal wall.

\begin{tabular}{lllll}
\hline Number Clusters & 2 & & & 5 \\
& & & & \\
\hline Num. Outliers Accepted & $2+$ & $3+$ & $4+$ & Scoring \\
\hline Septal Wall- All Patients & $0.61 \pm 0.08$ & $0.67 \pm 0.08$ & $0.64 \pm 0.08$ & $0.64 \pm 0.08$ \\
Septal Wall - Ischemic & $0.78 \pm 0.09$ & $0.81 \pm 0.08$ & $0.75 \pm 0.10$ & $0.82 \pm 0.08$ \\
Septal Wall - Non-Ischemic & $0.53 \pm 0.13$ & $0.67 \pm 0.12$ & $0.65 \pm 0.12$ & $0.55 \pm 0.13$ \\
& & & & \\
\hline
\end{tabular}

\subsubsection{SPECT RNA FA}

ROC AUC results for various FA parameters were previously calculated in the previous chapter (see section 4.3). The best results were obtained with septal $\mathrm{S}$ in the ischemic population where an AUC of 0.78 and an optimal sensitivity of $59 \%$ with $89 \%$ specificity was reported.

\subsubsection{PET scar size analysis}

The results from Birnie et al. (1) indicated that lateral wall scar size was predictive of CRT outcome in both ischemic and non-ischemic cardiomyopathy subsets of the PREDICT population. For ischemic patients, the ROC AUC was found to be $0.73 \pm 0.10$ with an optimal sensitivity/specificity of $76 \% / 67 \%$ at a cut-off scar size of $31.2 \%$. For non-ischemic patients, the ROC AUC was $0.75 \pm 0.10$ with an optimal operating point of $69 \%$ sensitivity and $78 \%$ specificity at a scar size cut-off of $5.6 \%$. 


\subsubsection{Data Analysis}

The best ROC curves obtained from cluster analysis ( $\mu \pm 2 \sigma$ outlier threshold, 2 clusters, $3+$ number of outliers for abnormal observation), FA (septal S), and lateral wall scar size are shown in Figure 5.3 for the ischemic population. Cluster analysis ROC AUC $(0.82 \pm 0.08)$ was not significantly greater than either FA analysis $(0.78 \pm 0.09 ; \mathrm{p}=0.74)$ or scar size analysis $(0.73 \pm 0.10 ; \mathrm{p}=0.48)$. At optimal operating points, cluster analysis concordance results $(\kappa=0.64 \pm 0.16)$ suggested an improved concordance in the ischemic group over SPECT RNA FA $(\kappa=0.41 \pm 0.19, \mathrm{p}=0.35$ vs. cluster analysis $)$ and PET scar size analysis $(\kappa=0.42 \pm 0.18 ; \mathrm{p}=0.37$ vs. cluster analysis), but the sample size was not large enough to show a significant difference.

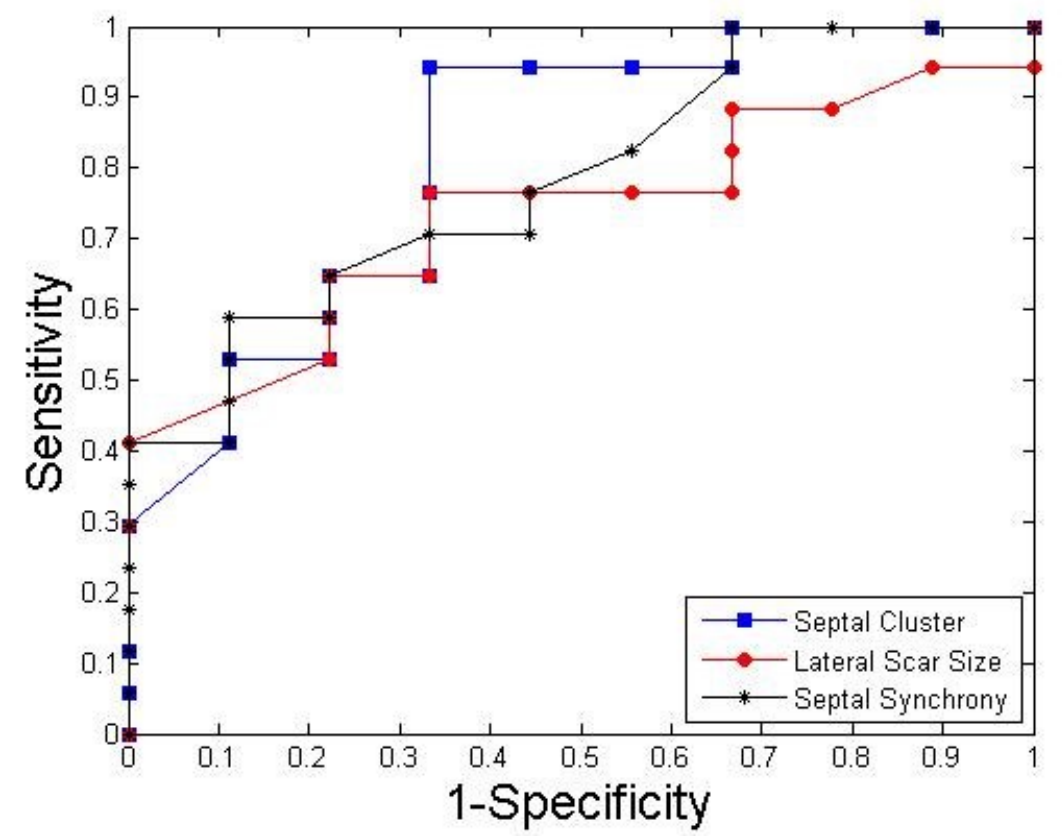

Figure 5.3: Comparison of ROC curves for the best results from cluster analysis, FA (septal synchrony), and lateral wall scar size. The optimal cluster classification was with 2 clusters and an abnormal TAC requiring $>=3$ point at $>=2$ standard deviations from normal. 


\subsection{Discussion}

In this study, a cluster analysis methodology was developed, and optimal settings for cluster analysis were determined. First, a database of 50 normal subjects was used to establish normal cardiac TACs representing normal cardiac contraction patterns. It was determined that any TAC sufficiently different from these normal reference curves would be identified as abnormal wall motion and thus labeled an abnormal curve. However, to properly compare TACs between patients, it was necessary to normalize the data. Three techniques, global, segmental and individual, were defined and investigated in order to minimize both the inter- and intra-subject variation. Unexpectedly, the inter-subject variability was insensitive to the normalization technique as it remained relatively constant (11-12\%), indicating that the mean TAC of each patient did not vary much with respect to another when varying normalization technique. However, as expected, the intra-subject variability improved considerably from $34 \%$ for global, to $25 \%$ for segmental to $15 \%$ for individual normalization. Following individual normalization, several metrics affecting the classification of a TAC were varied to determine the best combination for CRT outcome prediction.

The K-means clustering algorithm produced ROC AUC results of approximately 0.70 in the septal wall for all populations. ROC AUC results were 0.61 or lower in the global analysis and in the lateral wall (with the exception of the ischemic population). The poorer performance compared to the normal-average approach may be due to the nature of the automatic clustering process. With automatic clustering, it was impossible to pre- 
define which cluster represents the normal (or abnormal) cluster, and as such the smallest cluster in size was taken as the abnormal cluster. The greater the size of the smallest cluster, the more variability was present in the observations, suggesting more dyssynchrony. This works if one assumed that the majority of the LV is contracting synchronously. If this was not the case, the smallest cluster actually represented the small fraction that was contracting normally. In addition, the algorithm always separated observations into separate clusters even in the presence of minimal differences. No mechanism was implemented to alter the number of clusters or unify clusters presenting minimal differences. For example, all the observations of a patient with complete synchrony (or dyssynchrony) would still be forced into 2 clusters but not be meaningful as the classification would be based on noise. A potential solution would be to evaluate the final mean difference in the two clusters' centroids for the purpose of potentially unifying them if the difference was small enough. Deciding on a threshold difference for unification and subsequently classifying the unified cluster as normal or abnormal requires an independent measure of variability and 'normality'. This approach would thus end as something similar to the normal database approach.

The Euclidian distance was used as comparison for K-means algorithm. Another comparison metric may have produced better results, but exploration of this was not pursued in favor of evaluating the normal database alternative.

Clustering with a normal database produced a variety of results depending on the choice of parameters, though most ROC AUCs differences were not significant. There was a 
general trend that clustering with a normal database approach produced better results than K-means. In the majority of cases, accepting a $2 \sigma$ range around the mean TAC as normal produced the best results. Specifically, in the ischemic population, septal wall cluster analysis produced ROC AUC values ranging from 0.78 to 0.82 . Results using the $1 \sigma$ range included too many TACs into the abnormal cluster not allowing enough freedom for the natural variations between and within patients. Results at $3 \sigma$, on the other hand, provided too large a margin of uncertainty and the majority of patients had a $0 \%$ dyssynchrony value. Further refinement of the threshold to a value between $2 \sigma$ and $3 \sigma$ might yield additional small improvements in AUC and is a direction that may be pursued in the future. It was expected that a scoring system might produce better results as it would be possible to account for a greater impact of severely dyssynchronous sectors. This was not the case as results varied little between the 5 cluster scoring system and the 2 cluster size metric. Varying the number of outliers accepted before a TAC was classified as abnormal had a minor effect on the results. While keeping other metrics constant, the ROC AUC values in the majority of cases varied by 0.06 or less when varying the number of outliers accepted for a normal observation from 1 to 2 to 3 . Only the septal wall analysis of the non-ischemic population using a $\mu \pm 3 \sigma$ outlier threshold produced a greater difference than 0.06 between ROC AUC values for a different number of outlier metrics (0.14). None of the differences between the ROC AUC values were significantly different at the $95 \%$ confidence level. Concordance scores were also not significantly different in these comparisons $(\mathrm{p}>0.05)$. Despite the results not being statistically significant, 3 or more outliers before abnormal classification was chosen as 
the optimal metric setting since the concordance and ROC AUC tended to be equal or greater to the other possible combinations.

The major advantage of cluster analysis over FA is that it does not overgeneralize results by performing a first-order Fourier fit to a TAC, forcing a sinusoidal shape on the TAC. From this fit, only 2 parameters are extracted: phase and amplitude. Any abnormality in the shape of the curve is lost or only partially preserved and this likely affects a mechanical dyssynchrony patient more where abnormal contractions patterns are more prevalent. The retention of this additional information through the clustering approach may be an explanation for the improved performance observed.

Cluster analysis algorithms produced ROC AUC results for predicting CRT outcome on par with both SPECT RNA FA and PET scar size analysis. Cluster analysis provided more information on curve shape than FA and therefore it is not surprising that ROC AUC results tended to be greater, though not significantly different ( $p>0.05$ ), than FA for predicting CRT outcome. Furthermore, when observing optimal sensitivity and specificity values (Figure 5.3), cluster analysis of the septal wall in ischemic patients produced excellent sensitivity and specificity of $94 \%$ and $67 \%$ respectively. This value outperformed the best ROC AUC of any FA result (71\%/67\%) and scar analysis $(76 \% / 67 \%)$ also in the ischemic population. However, due to a relatively small sample size the superior concordance of cluster analysis was not significantly different from either FA or scar analysis ( $>0.05)$. 
In the ischemic population, cluster analysis successfully identified 4 responders and 1 non-responder that both scar size analysis and FA did not. Conversely, only 2 patients with scar size analysis and 1 with FA were properly identified but misclassified by cluster analysis. Interestingly, in scar size analysis, the lateral wall produced all significant results whereas in cluster analysis, the septal wall produced the best results. These observations suggest that different information is being used by cluster analysis and scar size analysis to assess the dyssynchrony of patients and that they may be partially complementary parameters in predicting CRT outcome.

In the majority of all clustering algorithms as well as in FA, septal wall analysis outperformed both global analysis and lateral wall analysis. This was a bit surprising as the majority of studies $(92,116,139)$ which have previously investigated CRT outcome using segmental analysis have reported that the lateral wall was the key segment that produced significant results. However, these studies used scar metrics to predict CRT outcome. The inferior results of global parameters suggest that some valuable information may be diluted by encompassing all values in a single global parameter.

A current limitation of cluster analysis is that the normal reference database is defined using an 8 gate SPECT RNA patient database. This limited the analysis of SPECT RNA data to patients with 8 gate scans. A normal subject database of 16 gate SPECT RNA data is required to properly analyze 16 gate SPECT patient data using cluster analysis. In addition, 16 or potentially 24 gates would better define the shape of the TAC curve and possibly do a better job of capturing this information. Increasing the number of gates 
will, however, increase the noise in the measurements which may degrade the quality of the information. Assessing the net benefit of increasing temporal resolution to 16 or 24 frames is a direction for future investigation.

Segmental analysis was limited to a 5 segment model. Increasing to a 9 or 17 segment model may provide improved accuracy of the normal reference TACs in each segment, but this would likely increase noise as the number of sectors per segment would be significantly decreased. It would possibly avoid the dilution in signal which can occur when averaging over an extended region and allow identification of smaller regions of abnormality. The clinical importance of identifying these smaller regions and optimal segment size needed for prediction of CRT response will require further investigation.

\subsection{Conclusion}

SPECT RNA cluster analysis algorithms were successfully developed and optimal settings for predicting CRT outcome were defined in a small test population. Cluster analysis produced an ROC AUC value and optimal sensitivity/specificity values that were at least equivalent to those from both FA and scar size. With cluster analysis, the septal wall segment produced the most predictive results, whereas the lateral wall provided the best information for scar size assessment suggesting that cluster analysis and scar size analysis may be partly complementary and thus provide additional information when used in conjunction. 


\section{Chapter 6}

\section{Discussion}

In this thesis, new methodologies were developed and validated using SPECT RNA to improve the quantification of MD with the ultimate goal of improving the ability of SPECT RNA to predict response to CRT. In chapter 3 FA amplitude values were compared to LV scar size in the lateral wall and it was determined that moderate but significant correlations existed between amplitude based parameters and scar. In chapter 4, novel MD parameters based on the amplitude component derived from FA were developed and evaluated alongside pre-established FA parameters for their ability at predicting CRT response. Results from the study indicated that septal wall A_SD, global $\mathrm{S}$ and global P_SD significantly predicted CRT response. Finally, in chapter 5, a novel methodology called cluster analysis was developed and optimized to evaluate its ability as an MD parameter to predict response to CRT and determine if it could improve on existing FA results. Similar to chapter 4, significant results occurred in the septal wall for predicting response to CRT. The results of cluster analysis were then compared to the best results obtained from FA (septal S) and PET scar size in the same population. Despite producing a greater ROC AUC and an improved sensitivity and specificity 
operating point, cluster analysis was not significantly better than lateral wall scar size or septal S ( $>0.05)$.

In this thesis, novel SPECT RNA methodologies provided improved quantification of MD in CHF patients. For the first time, the septal wall was shown to be a key segment in predicting response to CRT in both FA amplitude analysis and cluster analysis. This is different from prevailing thought and is contrary to several scar studies which showed that lateral wall was the important segment $(1,92,116,122)$ These results suggest that the septal wall should be a consideration in further wall motion analysis.

It was shown that FA amplitude-based parameters, alone or in conjunction with phase values (synchrony) are significant predictors of CRT response, equivalent to both phasebased FA parameters values and scar size. However, the moderate correlation of amplitude with scar indicated that other factors influence amplitude and that it is not a suitable surrogate marker of scar. Furthermore, phase values were associated with hospitalization outcomes while amplitude MD parameters were predictive of death. This unexpected result suggests that the different mechanisms of progression of CHF may affect FA amplitude and phase values differently.

Using the full shape of the TAC, cluster analysis assessed MD patterns and successfully discriminated between normal and abnormal wall motion curves. This novel methodology produced results that suggested improved performance over scar and FA for the prediction of CRT response, but the difference was not significant. 
The statistically significant ROC AUC results obtained in this thesis for predicting CRT response ranged from 0.66 to 0.82 . These modest AUC values suggest that a single MD parameter may not be sufficient to predict CRT with high accuracy. However, several of the parameters investigated in this thesis may be partially complementary. Although summary measures, such as the total number of correctly identified responder patients, were largely equivalent between the different MD parameters, the specific patients identified were not identical. This suggests that a combination of MD parameters might produce incremental improvement over single parameters alone.

\subsection{Limitations and future directions}

A limitation of this thesis is that it did not investigate any multivariate models that combine FA parameters, cluster analysis and/or scar metrics. This is the next step that should be performed in this research to determine if a significant improvement in the prediction of CRT response can be achieved.

The patient populations used in this thesis were relatively small which limited the power of the statistical tests performed. Larger patient populations would help minimize the standard deviation in populations and help determine if differences observed between groups or MD parameters are real. However, large patient populations who have undergone SPECT RNA are not often readily available. In the multicenter RAFT study, 1798 patients were enrolled but only 214 obtained SPECT RNA scans. 
Results in this thesis have indicated a benefit to performing LV segmental analysis. In this thesis, segmental analysis was performed on a 5 segment model. Smaller region sizes may help with the localization of MD sectors possibly at the cost of increased noise. Various sizes of LV segments, such as 9 or 17 segment models could be investigated to determine optimal region size. This could help improve the ability of FA parameters at predicting CRT response and warrants further investigation.

Cluster analysis, which produced the best individual ROC result, is currently limited to analysis of patients who underwent SPECT RNA scans ECG-gated to 8 gates. Increasing the number of gates would permit better sampling of the wall motion curve shape but potentially increases the noise of the image. Employing new dedicated cardiac SPECT cameras would offer an increase in sensitivity over traditional systems which would help reduce noise. To increase the sampling rate, a normal patient database of 16 or 24 gates would be required to develop the normal cardiac wall motion curves. Improved temporal resolution may provide better information and new insights into wall-motion abnormalities.

Inherent limitations exist when developing a diagnostic tool for predicting response to CRT. The intervention itself is not perfect and pacemaker leads may not be positioned at ideal sites as outlined in section 1.3.1. The CRT response criteria can vary from study to study and can create significantly different responder and non-responder populations. Both these factors affect the perceived ability of MD to predict response. Exclusion of patients with improper lead placements may improve the ability of the novel SPECT 
RNA diagnostic tool to predict response to CRT. Information on location of lead placement was not available in the patient populations used in this thesis.

A SPECT RNA tool that allows the quantification of novel MD parameters was developed and produced promising results at understanding the problem of non-response. Further clinical evaluations, such as a prospective study using the novel MD parameters established in this thesis, could provide additional insight on the relationship between MD and CRT non-response.

Another possible direction for this research is to identify other possible applications where MD quantification may provide further diagnostic assistance. SPECT RNA scans are often used to qualitatively assess the cardiotoxicity of chemotherapy patients. A quantitative evaluation of MD to assess changes in MD may provide earlier assessment of cardiotoxicity than current methods of $\triangle \mathrm{LVEF}$ and visual assessment, allowing for quicker intervention. Other potential applications may include improving the evaluation of various arrhythmias such as ventricular tachycardia, aneurysms and any other cardiac conditions where abnormal contraction patterns may be present. 


\subsection{Conclusion}

Novel MD parameters were successfully developed and optimized and were shown to be statistically significant for predicting response to CRT. Amplitude-based FA parameters correlated only moderately with scar but were nonetheless predictors of CRT response equivalent to existing FA parameters and scar analysis results. Cluster analysis produced the best individual results for predicting response to CRT, but the results were not significantly better than FA or scar analysis. This suggests that cluster analysis may be the most promising method for MD quantification and predicting response to CRT. Regional analysis of the septal wall was the only segment to provide significant results in both FA and cluster analysis outperforming other regional segments as well as global MD parameters. 


\section{Appendix A}

\section{Copyright Permission}

Copyright permission was obtained for Figure 1.3 and is provided on the next page. All other figures presented in this thesis were the author's original work or were obtained from the public domain. 
Dear Michel Lalonde

We hereby grant you permission to reprint the material detailed below at no charge in your thesis subject to the following conditions:

1. If any part of the material to be used (for example, figures) has appeared in our publication with credit or acknowledgement to another source, permission must also be sought from that source. If such permission is not obtained then that material may not be included in your publication/copies.

2. Suitable acknowledgment to the source must be made, either as a footnote or in a reference list at the end of your publication, as follows:

"This article was published in Publication title, Vol number, Author(s), Title of article, Page Nos, Copyright Elsevier (or appropriate Society name) (Year)."

3. Your thesis may be submitted to your institution in either print or electronic form.

4. Reproduction of this material is confined to the purpose for which permission is hereby given.

5. This permission is granted for non-exclusive world English rights only. For other languages please reapply separately for each one required. Permission excludes use in an electronic form other than submission. Should you have a specific electronic project in mind please reapply for permission

6. This includes permission for the Library and Archives of Canada to supply single copies, on demand, of the complete thesis. Should your thesis be published commercially, please reapply for permission.

Yours sincerely

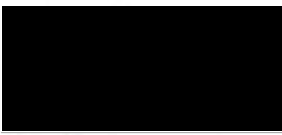

Jennifer Jones

Rights Associate

Elsevier Limited, a company registered in England and Wales with company number 1982084, whose registered office is The Boulevard, Langford Lane, Kidlington, Oxford, OX5 1GB, United Kingdom.

From: mlalonde15@rogers.com [mailto:mlalonde15@rogers.com]

Sent: 06 May 2013 15:35

To: Rights and Permissions (ELS) 
Subject: Obtain Permission - Book request

Title:

First name:

Last name:

Institute/company:

Address:

Post/Zip Code:

City:

Country:

Telephone:

Email:

Please select the type of publication:

Book - Title:

Book - ISBN:

Book - Author(s):

Book - Year:

Book - Pages from:

Book - Pages to:

Book - Chapter Num:

Book - Chapter Title:

I would like to use (please select one of the following options):

If using figures/tables or illustrations please specify the quantity:

Are you the author of the material?:

If not, is the author involved with your project:

In what format will you use the material?:

Will you be translating the material?:

Information about your proposed use:

Proposed use text:
Mr.

Michel

Lalonde

Carleton University

1578 Henri Lauzon

K1C7R8

Ottawa

Canada

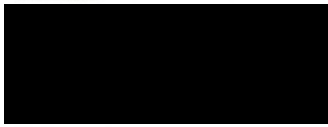

Book

ACLS Study Guide

978-0-323-04695-4

B. Aehlert

2007

127

127

3

Rhythm Recognition

Figures(s)

1

No

No

Print

No

thesis

Non-Exclusive License with Library and Archives

Canada

Elsevier Limited. Registered Office: The Boulevard, Langford Lane, Kidlington, Oxford, OX5 1GB, United Kingdom, Registration No. 1982084, Registered in England and Wales. 


\section{References}

1. Birnie D, deKemp RA, Ruddy TD, et al. Effect of lateral wall scar on reverse remodeling with cardiac resynchronization therapy. Heart Rhythm. 2009;6:1721-1726.

2. Tang ASL, Wells GA, Talajic M, et al. Cardiac-resynchronization therapy for mild-tomoderate heart failure. $N$ Engl J Med. 2010;363:2385-2395.

3. Tang AS. Resynchronization/defibrillation for ambulatory heart failure trial: Rationale and trial design. Curr Opin Cardiol. 2009;24:1-8.

4. Http://www.sciencekids.co.nz/. Science Kids. February 25, 2013;April 10, 2013.

5. Aehlert B. ACLS Study Guide. Third Edition ed. Mosby Jems, Elsevier; 2007:592.

6. Surawicz B. AHA/ACCF/HRS recommendations for the standardization and interpretation of the electrocardiogram: Part III: Intraventricular conduction disturbances: A scientific statement from the american heart association electrocardiography and arrhythmias committee, council on clinical cardiology the american college of cardiology foundation and the heart rhythm society. Circulation. 2009;119:e235-e240. 
7. Leclercq C. Systolic improvement and mechanical resynchronization does not require electrical synchrony in the dilated failing heart with left bundle-branch block. Circulation. 2002;106:1760-1763.

8. Yu C-. High prevalence of left ventricular systolic and diastolic asynchrony in patients with congestive heart failure and normal QRS duration. Heart. 2003;89:54-60.

9. Bleeker GB. Relationship between QRS duration and left ventricular dyssynchrony in patients with end-stage heart failure. J Cardiovasc Electrophysiol. 2004;15:544-549.

10. Kass DA. Predicting cardiac resynchronization response by QRS duration: The long and short of it. J Am Coll Cardiol. 2003;42:2125-2127.

11. Grines CL. Functional abnormalities in isolated left bundle branch block. the effect of interventricular asynchrony. Circulation. 1989;79:845-853.

12. Bax JJ. Echocardiographic evaluation of cardiac resynchronization therapy: Ready for routine clinical use? A critical appraisal. J Am Coll Cardiol. 2004;44:1-9.

13. Kass DA. Retiming the failing heart: Principles and current clinical status of cardiac resynchronization. J Am Coll Cardiol. 2002;39:194-201.

14. Ghio S. Interventricular and intraventricular dyssynchrony are common in heart failure patients, regardless of QRS duration. Eur Heart J. 2004;25:571-578.

15. Marieb E. Essentials of Human Anatomy and Physiology. 10th ed. Pearson; 2012:632. 
16. The Criteria Committee of the New York Heart Association. Nomenclature and Criteria for Diagnosis of Diseases of the Heart and Great Vessels. 9th ed. Boston, MA: Little, Brown and Co.; 1994.

17. Public Health Agency of Canada. 2009 tracking heart disease and stroke in canada. Canada: Public Health Agency of Canada; 2009.

18. Chronic disease infobase data cubes. Public Health Agency of Canada. 2012;May 10, 2013. Available from: http://66.240.150.17/cubes/data-cubes-eng.html.

19. Abraham WT, Fisher WG, Smith AL, et al. Cardiac resynchronization in chronic heart failure. $N$ Engl J Med. 2002;346:1845-1853.

20. Cazeau S. Effects of multisite biventricular pacing in patients with heart failure and intraventricular conduction delay. $N$ Engl J Med. 2001;344:873-80.

21. Cleland JGF. The CARE-HF study (cardiac resynchronisation in heart failure study): Rationale, design and end-points. European journal of heart failure. 2001;3:481-489.

22. Cleland JGF. The effect of cardiac resynchronization on morbidity and mortality in heart failure. $N$ Engl J Med. 2005;352:1539-49.

23. Bristow MR. Heart failure management using implantable devices for ventricular resynchronization: Comparison of medical therapy, pacing, and defibrillation in chronic heart failure (COMPANION) trial. J Card Fail. 2000;6:276-285. 
24. Bristow MR. Cardiac-resynchronization therapy with or without an implantable defibrillator in advanced chronic heart failure. N Engl J Med. 2004;350:2140-2150 2227.

25. Moss AJ. Multicenter automatic defibrillator implantation trial-cardiac resynchronization therapy (MADIT-CRT): Design and clinical protocol. Annals of noninvasive electrocardiology. 2005;10:34-43.

26. Moss AJ. Cardiac-resynchronization therapy for the prevention of heart-failure events. N Engl J Med. 2009;361:1329-1338.

27. Tracy CM. 2012 ACCF/AHA/HRS focused update of the 2008 guidelines for devicebased therapy of cardiac rhythm abnormalities: A report of the american college of cardiology foundation/american heart association task force on practice guidelines. Circulation. 2012;126:1784-1800.

28. Stevenson WG. Indications for cardiac resynchronization therapy: 2011 update from the heart failure society of america guideline committee. J Card Fail. 2012;18:94-106.

29. Exner DV. Canadian cardiovascular society guidelines on the use of cardiac resynchronization therapy: Evidence and patient selection. Can J Cardiol. 2013;29:182195.

30. Cleland JGF, Calvert MJ, Verboven Y, Freemantle N. Effects of cardiac resynchronization therapy on long-term quality of life: An analysis from the CArdiac resynchronisation-heart failure (CARE-HF) study. Am Heart J. 2009;157:457-466. 
31. McAlister FA, Ezekowitz J, Dryden DM, et al. Cardiac resynchronization therapy and implantable cardiac defibrillators in left ventricular systolic dysfunction. Evidence report/technology assessment. 2007:1-199.

32. Rivero-Ayerza M, Theuns DAMJ, Garcia-Garcia HM, Boersma E, Simoons M, Jordaens LJ. Effects of cardiac resynchronization therapy on overall mortality and mode of death: A meta-analysis of randomized controlled trials. Eur Heart J. 2006;27:26822688.

33. Birnie DH, Tang AS. The problem of non-response to cardiac resynchronization therapy. Curr Opin Cardiol. 2006;21:20-26.

34. Chung ES, Leon AR, Tavazzi L, et al. Results of the predictors of response to CRT (PROSPECT) trial. Circulation. 2008;117:2608-2616.

35. Young JB. Combined cardiac resynchronization and implantable cardioversion defibrillation in advanced chronic heart failure: The MIRACLE ICD trial. JAMA: the Journal of the American Medical Association. 2003;289:2685-2694.

36. Yu C-, Bleeker GB, Fung JW-, et al. Left ventricular reverse remodeling but not clinical improvement predicts long-term survival after cardiac resynchronization therapy. Circulation. 2005;112:1580-1586.

37. Bader H. Intra-left ventricular electromechanical asynchrony: A new independent predictor of severe cardiac events in heart failure patients. J Am Coll Cardiol. 2004;43:248-256. 
38. Yang H. Regional left ventricle mechanical asynchrony in patients with heart disease and normal QRS duration: Implication for biventricular pacing therapy. Pacing and clinical electrophysiology. 2003;26:562-570.

39. Padeletti L, Paoletti Perini A, Gronda E. Cardiac resynchronization therapy: The issue of non-response. Heart Fail Rev. 2012;17:97-105.

40. Auricchio A, Regoli F. Past, present, and future of CRT. Heart Fail Rev. 2011;16:205-214.

41. Botvinick EH, William O'Connell J, Badhwar N. Editorial: Imaging synchrony. Journal of Nuclear Cardiology. 2009;16:846-848.

42. Vardas PE, Auricchio A, Blanc J-, et al. Guidelines for cardiac pacing and cardiac resynchronization therapy: The task force for cardiac pacing and cardiac resynchronization therapy of the european society of cardiology. developed in collaboration with the european heart rhythm association. Eur Heart J. 2007;28:22562295.

43. Ansalone G. Doppler myocardial imaging in patients with heart failure receiving biventricular pacing treatment. The American heart journal. 2001;142:881-896.

44. Ansalone G. Doppler myocardial imaging to evaluate the effectiveness of pacing sites in patients receiving biventricular pacing. $J$ Am Coll Cardiol. 2002;39:489-499. 
45. O'Mara JE. Regional patterns of dyssynchrony: Lateral wall delay is desirable but not essential for left ventricular remodeling in biventricular pacing. Echocardiography. 2012;29:554-559.

46. Thebault C. Sites of left and right ventricular lead implantation and response to cardiac resynchronization therapy observations from the REVERSE trial. Eur Heart J. 2012;33:2662-2671.

47. Ypenburg C. Optimal left ventricular lead position predicts reverse remodeling and survival after cardiac resynchronization therapy. J Am Coll Cardiol. 2008;52:1402-1409.

48. Merchant FM. Impact of segmental left ventricle lead position on cardiac resynchronization therapy outcomes. Heart rhythm. 2010;7:639-644.

49. Murphy RT. Tissue synchronization imaging and optimal left ventricular pacing site in cardiac resynchronization therapy. Am J Cardiol. 2006;97:1615-1621.

50. Cho GY, Kim MJ, Park JH, et al. Comparison of ventricular dyssynchrony according to the position of right ventricular pacing electrode: A multi-center prospective echocardiographic study. J Cardiovasc Ultrasound. 2011;19:15-20.

51. Prince JL, Links JM. Medical Imaging Signals and Systems. Pearson; 2006:480.

52. Krane KS, Halliday D. Introductory Nuclear Physics. New York: Wiley; 1987:845.

53. Nuclear decay data in the MIRD format, URL: Http://www.nndc.bnl.gov/mird/. National Nuclear Data Center. ;2013. 
54. Saha GB. Fundamentals of Nuclear Pharmacy. 5th ed. New York: Springer; 2004:383.

55. Callahan RJ, Froelich JW, McKusick KA. A modified method for the in vivo labeling of red blood cells with tc-99m: Concise communication. Journal of Nuclear Medicine. 1982;23:315-318.

56. Lilley J. Nuclear Physics : Principles and Applications. Chichester: J. Wiley; 2001.

57. Fernow R. Introduction to Experimental Particle Physics. Cambridge, Cambridgeshire: Cambridge University Press; 1986.

58. Werwick M, Aarsvold J. Emission Tomography: The Fundamentals of PET and SPECT. Academic Press; 2004:596.

59. Ramachandran GN. Three-dimensional reconstruction from radiographs and electron micrographs: Application of convolutions instead of fourier transforms. Proc Natl Acad Sci U S A. 1971;68:2236-2240.

60. Lange K. EM reconstruction algorithms for emission and transmission tomography. $J$ Comput Assist Tomogr. 1984;8:306-316.

61. Cerqueira MD, Weissman NJ, Dilsizian V, et al. Standardized myocardial segmentation and nomenclature for tomographic imaging of the heart. A statement for healthcare professionals from the cardiac imaging committee of the council on clinical cardiology of the american heart association. Int J Cardiovasc Imaging. 2002;18:539542. 
62. Port SC. Imaging guidelines for nuclear cardiology procedures. part 2. Journal of nuclear cardiology1999;6.

63. Bonow RO. Standardization of cardiac tomographic imaging. Circulation. 1992;86:338-339.

64. Chen J. Temporal resolution of multiharmonic phase analysis of ECG-gated myocardial perfusion SPECT studies. Journal of nuclear cardiology. 2008;15:383-391.

65. Lalonde M, Birnie D, Ruddy TD, deKemp RA, Wassenaar RW. SPECT blood pool phase analysis can accurately and reproducibly quantify mechanical dyssynchrony. $J$ Nucl Cardiol. 2010;17:803-810.

66. Links JM, Douglass KH, Wagner Jr. HN. Patterns of ventricular emptying by fourier analysis of gated blood-pool studies. Journal of Nuclear Medicine. 1980;21:978-982.

67. Botvinick E, Dunn R, Frais M, et al. The phase image: Its relationship to patterns of contraction and conduction. Circulation. 1982;65:551-560.

68. O'Connell JW, Schreck C, Moles M, et al. A unique method by which to quantitate synchrony with equilibrium radionuclide angiography. J Nucl Cardiol. 2005;12:441-450.

69. Wassenaar R. Optimization and validation of radionuclide angiography phase analysis parameters for quantification of mechanical dyssynchrony. Journal of nuclear cardiology. 2009; 16:895-903. 
70. Le Rest C, Couturier O, Turzo A, et al. Use of left ventricular pacing in heart failure: Evaluation by gated blood pool imaging. Journal of Nuclear Cardiology. 1999;6:651656.

71. Kerwin WF. Ventricular contraction abnormalities in dilated cardiomyopathy: Effect of biventricular pacing to correct interventricular dyssynchrony. J Am Coll Cardiol. 2000;35:1221-1227.

72. Toussaint J-, Lavergne T, Kerrou K, et al. Basal asynchrony and resynchronization with biventricular pacing predict long-term improvement of LV function in heart failure patients. PACE - Pacing and Clinical Electrophysiology. 2003;26:1815-1823.

73. Botvinick EH, Badhwar N, O'Connell JW. Cardiac resynchronization therapy: The role of equilibrium radionuclide angiography. Medicamundi. 2008;52:51-58.

74. Fauchier L, Marie O, Casset-Senon D, Babuty D, Cosnay P, Fauchier JP. Interventricular and intraventricular dyssynchrony in idiopathic dilated cardiomyopathy: A prognostic study with fourier phase analysis of radionuclide angioscintigraphy. $\mathrm{J} \mathrm{Am}$ Coll Cardiol. 2002;40:2022-2030.

75. Hanley JA. The meaning and use of the area under a receiver operating characteristic (ROC) curve. Radiology. 1982;143:29-36.

76. Zweig MH. Receiver-operating characteristic (ROC) plots: A fundamental evaluation tool in clinical medicine. Clin Chem. 1993;39:561-577.

77. Fawcett T. An introduction to ROC analysis. Pattern Recog Lett. 2006;27:861-874. 
78. Botvinick EH. An accurate means of detecting and characterizing abnormal patterns of ventricular activation by phase image analysis. Am J Cardiol. 1982;50:289-298.

79. Frais M, Botvinick E, Shosa D, et al. Phase image characterization of localized and generalized left ventricular contraction abnormalities. J Am Coll Cardiol. 1984;4:987998.

80. Botvinick EH. Scintigraphic blood pool and phase image analysis: The optimal tool for the evaluation of resynchronization therapy. J Nucl Cardiol. 2003;10:424-428.

81. Casset-Senon D, Babuty D, Philippe L, et al. Fourier phase analysis of SPECT equilibrium radionuclide angiography in symptomatic patients with mitral valve prolapse without significant mitral regurgitation: Assessment of biventricular functional abnormalities suggesting a cardiomyopathy. J Nucl Cardiol. 2000;7:471-477.

82. Muramatsu T, Matsumoto K, Nishimura S. Efficacy of the phase images in fourier analysis using gated cardiac POOL-SPECT for determining the indication for cardiac resynchronization therapy. Circ J. 2005;69:1521-1526.

83. Harel F, Finnerty V, Gregoire J, Thibault B, Khairy P. Comparison of left ventricular contraction homogeneity index using SPECT gated blood pool imaging and planar phase analysis. J Nucl Cardiol. 2008;15:80-85.

84. Badhwar N, O'Connell J, Green D, DeMarco T, Botvinick E. Equilibrium radionuclide angiogram derived measures of dyssynchrony correlate with clinical outcomes in heart failure patients. $J$ Nucl Med Meeting Abstracts. 2008;49:128P-c. 
85. Badhwar N, O'Connell J, Green D, DeMarco T, Botvinick E. The value of SPECT equilibrium radionuclide angiogram synchrony analysis. J Nucl Med Meeting Abstacts. 2008;49:192P-b.

86. Chen J, Garcia EV, Folks RD, et al. Onset of left ventricular mechanical contraction as determined by phase analysis of ECG-gated myocardial perfusion SPECT imaging: Development of a diagnostic tool for assessment of cardiac mechanical dyssynchrony. $J$ Nucl Cardiol. 2005;12:687-695.

87. Trimble MA. Evaluation of left ventricular mechanical dyssynchrony as determined by phase analysis of ECG-gated SPECT myocardial perfusion imaging in patients with left ventricular dysfunction and conduction disturbances. Journal of nuclear cardiology. 2007;14:298-307.

88. Van Kriekinge SD, Nishina H, Ohba M, Berman DS, Germano G. Automatic global and regional phase analysis from gated myocardial perfusion SPECT imaging: Application to the characterization of ventricular contraction in patients with left bundle branch block. Journal of Nuclear Medicine. 2008;49:1790-1797.

89. Henneman MM, Chen J, Dibbets-Schneider P, et al. Can LV dyssynchrony as assessed with phase analysis on gated myocardial perfusion SPECT predict response to CRT? J Nucl Med. 2007;48:1104-1111.

90. Boogers MM, Van Kriekinge SD, Henneman MM, et al. Quantitative gated SPECTderived phase analysis on gated myocardial perfusion SPECT detects left ventricular 
dyssynchrony and predicts response to cardiac resynchronization therapy. Journal of Nuclear Medicine. 2009;50:718-725.

91. Adelstein EC, Tanaka H, Soman P, et al. Impact of scar burden by single-photon emission computed tomography myocardial perfusion imaging on patient outcomes following cardiac resynchronization therapy. Eur Heart J. 2011;32:93-103.

92. Ypenburg C, Schalij MJ, Bleeker GB, et al. Impact of viability and scar tissue on response to cardiac resynchronization therapy in ischaemic heart failure patients. Eur Heart J. 2007;28:33-41.

93. Cheung A, Zhou Y, Faber TL, Garcia EV, Zhu L, Chen J. The performance of phase analysis of gated SPECT myocardial perfusion imaging in the presence of perfusion defects: A simulation study. Journal of Nuclear Cardiology. 2012;19:500-506.

94. Pitzalis MV. Cardiac resynchronization therapy tailored by echocardiographic evaluation of ventricular asynchrony. J Am Coll Cardiol. 2002;40:1615-1622.

95. Petrovic M. Prediction of a good response to cardiac resynchronization therapy in patients with severe dilated cardyomyopathy: Could conventional echocardiography be the answer after all? Echocardiography. 2012;29:267-275.

96. Ho CY. A clinician's guide to tissue doppler imaging. Circulation. 2006;113:e396e398.

97. Abraham TP. Role of tissue doppler and strain echocardiography in current clinical practice. Circulation. 2007;116:2597-2609. 
98. Mondillo S. Speckle-tracking echocardiography: A new technique for assessing myocardial function. Journal of ultrasound in medicine. 2011;30:71-83.

99. Bax JJ. Left ventricular dyssynchrony predicts benefit of cardiac resynchronization therapy in patients with end-stage heart failure before pacemaker implantation. Am J Cardiol. 2003;92:1238-1240.

100. Bax JJ. Left ventricular dyssynchrony predicts response and prognosis after cardiac resynchronization therapy. J Am Coll Cardiol. 2004;44:1834-1840.

101. Notabartolo D. Usefulness of the peak velocity difference by tissue doppler imaging technique as an effective predictor of response to cardiac resynchronization therapy. Am J Cardiol. 2004;94:817-820.

102. Penicka M, Bartunek J, De Bruyne B, et al. Improvement of left ventricular function after cardiac resynchronization therapy is predicted by tissue doppler imaging echocardiography. Circulation. 2004;109:978-983.

103. Porciani MC, Cappelli F, Perrotta L, et al. Has mechanical dyssynchrony still a role in predicting cardiac resynchronization therapy response. Echocardiography. 2010;27:831-838.

104. Sogaard P. Tissue doppler imaging predicts improved systolic performance and reversed left ventricular remodeling during long-term cardiac resynchronization therapy. J Am Coll Cardiol. 2002;40:723-730. 
105. Altman RK. Usefulness of low-dose dobutamine echocardiography to predict response and outcome in patients undergoing cardiac resynchronization therapy. Am $J$ Cardiol. 2011;108:252-257.

106. Yu C-. Tissue doppler imaging is superior to strain rate imaging and postsystolic shortening on the prediction of reverse remodeling in both ischemic and nonischemic heart failure after cardiac resynchronization therapy. Circulation. 2004;110:66-73.

107. Risum N, Jons C, Olsen NT, et al. Simple regional strain pattern analysis to predict response to cardiac resynchronization therapy: Rationale, initial results, and advantages. Am Heart J. 2012;163:697-704.

108. Suffoletto MS. Novel speckle-tracking radial strain from routine black-and-white echocardiographic images to quantify dyssynchrony and predict response to cardiac resynchronization therapy. Circulation. 2006;113:960-968.

109. Gorcsan J. Combined longitudinal and radial dyssynchrony predicts ventricular response after resynchronization therapy. J Am Coll Cardiol. 2007;50:1476-1483.

110. Yu CM, Abraham WT, Bax J, et al. Predictors of response to cardiac resynchronization therapy (PROSPECT)--study design. Am Heart J. 2005;149:600-605.

111. Lardo AC. Magnetic resonance imaging assessment of ventricular dyssynchrony: Current and emerging concepts. J Am Coll Cardiol. 2005;46:2223-2228.

112. Sa MI. Imaging techniques in cardiac resynchronization therapy. The international journal of cardiovascular imaging. 2008;24:89-105. 
113. Westenberg JJM, Lamb HJ, van der Geest RJ, et al. Assessment of left ventricular dyssynchrony in patients with conduction delay and idiopathic dilated cardiomyopathy. head-to-head comparison between tissue doppler imaging and velocity-encoded magnetic resonance imaging. J Am Coll Cardiol. 2006;47:2042-2048.

114. Russel IK. Mechanical dyssynchrony or myocardial shortening as MRI predictor of response to biventricular pacing? Journal of magnetic resonance imaging. 2007;26:14521460.

115. White JA, Yee R, Yuan X, et al. Delayed enhancement magnetic resonance imaging predicts response to cardiac resynchronization therapy in patients with intraventricular dyssynchrony. J Am Coll Cardiol. 2006;48:1953-1960.

116. Chalil S, Stegemann B, Muhyaldeen SA, et al. Effect of posterolateral left ventricular scar on mortality and morbidity following cardiac resynchronization therapy. PACE - Pacing and Clinical Electrophysiology. 2007;30:1201-1209.

117. Chalil S, Foley PWX, Muyhaldeen SA, et al. Late gadolinium enhancementcardiovascular magnetic resonance as a predictor of response to cardiac resynchronization therapy in patients with ischaemic cardiomyopathy. Europace. 2007;9:1031-1037.

118. Ypenburg C. Effect of total scar burden on contrast-enhanced magnetic resonance imaging on response to cardiac resynchronization therapy. Am J Cardiol. 2007;99:657660. 
119. Jansen AHM, Bracke F, Van Dantzig JM, et al. The influence of myocardial scar and dyssynchrony on reverse remodeling in cardiac resynchronization therapy. European Journal of Echocardiography. 2008;9:483-488.

120. Taylor AJ, Elsik M, Broughton A, et al. Combined dyssynchrony and scar imaging with cardiac magnetic resonance imaging predicts clinical response and long-term prognosis following cardiac resynchronization therapy. Europace. 2010;12:708-713.

121. Petryka J. Magnetic resonance imaging assessment of intraventricular dyssynchrony and delayed enhancement as predictors of response to cardiac resynchronization therapy in patients with heart failure of ischaemic and non-ischaemic etiologies. Eur J Radiol. 2012;81:2639-2647.

122. Marsan NA, Westenberg JJM, Ypenburg C, et al. Magnetic resonance imaging and response to cardiac resynchronization therapy: Relative merits of left ventricular dyssynchrony and scar tissue. Eur Heart J. 2009;30:2360-2367.

123. Bilchick KC, Dimaano V, Wu KC, et al. Cardiac magnetic resonance assessment of dyssynchrony and myocardial scar predicts function class improvement following cardiac resynchronization therapy. JACC: Cardiovascular Imaging. 2008;1:561-568.

124. Pazhenkottil AP. Left ventricular dyssynchrony assessment by phase analysis from gated PET-FDG scans. Journal of nuclear cardiology. 2011;18:920-925. 
125. Uebleis C. Electrocardiogram-gated 18F-FDG PET/CT hybrid imaging in patients with unsatisfactory response to cardiac resynchronization therapy: Initial clinical results. The Journal of Nuclear Medicine. 2011;52:67-71.

126. Birnie D, de Kemp RA, Tang AS, et al. Reduced septal glucose metabolism predicts response to cardiac resynchronization therapy. Journal of Nuclear Cardiology. 2011:111.

127. Beanlands RSB, Nichol G, Huszti E, et al. F-18-fluorodeoxyglucose positron emission tomography imaging-assisted management of patients with severe left ventricular dysfunction and suspected coronary disease. A randomized, controlled trial (PARR-2). J Am Coll Cardiol. 2007;50:2002-2012.

128. Beanlands RSB, Ruddy TD, DeKemp RA, et al. Positron emission tomography and recovery following revascularization (PARR-1): The importance of scar and the development of a prediction rule for the degree of recovery of left ventricular function. $J$ Am Coll Cardiol. 2002;40:1735-1743.

129. Vitale GD, Dekemp RA, Ruddy TD, Williams K, Beanlands RSB. Myocardial glucose utilization and optimization of 18F-FDG PET imaging in patients with noninsulin-dependent diabetes mellitus, coronary artery disease, and left ventricular dysfunction. Journal of Nuclear Medicine. 2001;42:1730-1736.

130. Anselm DD, Anselm AH, Renaud J, et al. Altered myocardial glucose utilization and the reverse mismatch pattern on rubidium-82 perfusion/F-18-FDG PET during the 
sub-acute phase following reperfusion of acute anterior myocardial infarction. Journal of Nuclear Cardiology. 2011;18:657-667.

131. Schelbert HR, Beanlands R, Bengel F, et al. PET myocardial perfusion and glucose metabolism imaging: Part 2 - guidelines for interpretation and reporting. Journal of Nuclear Cardiology. 2003;10:557-571.

132. Masc RK, Renaud JM, Ziadi MC, et al. Intra-and inter-operator repeatability of myocardial blood flow and myocardial flow reserve measurements using rubidium- 82 pet and a highly automated analysis program. Journal of Nuclear Cardiology. 2010;17:600616.

133. Klein R, Lortie M, Adler A, Beanlands R, deKemp R. Fully automated software for polar-map registration and sampling from PET images. IEEE Nucl Sci Symp Conf Rec. 2006;6:3185.

134. Lee F. Rapid radionuclide-derived systolic and diastolic cardiac function using cycle-dependent backsground correction and fourier analysis. Long Beach, Calif.: IEEE Computer Society; 1985:443-446.

135. Massardo T, Gal RA, Grenier RP, Schmidt DH, Port SC. Left ventricular volume calculation using a count-based ratio method applied to multigated radionuclide angiography. Journal of Nuclear Medicine. 1990;31:450-456.

136. Grizzard JD, Judd RM, Kim RJ. Ischemic heart disease and non-ischemic cardiomyopathies. In: Cardiovascular MRI in Practice. Springer London; 2008:25-40. 
137. Gottlieb I, Macedo R, Bluemke DA, Lima JAC. Magnetic resonance imaging in the evaluation of non-ischemic cardiomyopathies: Current applications and future perspectives. Heart Fail Rev. 2006;11:313-323.

138. Assomull RG, Prasad SK, Lyne J, et al. Cardiovascular magnetic resonance, fibrosis, and prognosis in dilated cardiomyopathy. J Am Coll Cardiol. 2006;48:19771985.

139. Bleeker GB, Kaandorp TAM, Lamb HJ, et al. Effect of posterolateral scar tissue on clinical and echocardiographic improvement after cardiac resynchronization therapy. Circulation. 2006;113:969-976.

140. Chen J, Garcia EV, Bax JJ, Iskandrian AE, Borges-Neto S, Soman P. SPECT myocardial perfusion imaging for the assessment of left ventricular mechanical dyssynchrony. Journal of Nuclear Cardiology. 2011;18:685-694.

141. Ludwig DR. On the importance of image gating for the assay of left ventricular mechanical dyssynchrony using SPECT. The Journal of Nuclear Medicine. 2012;53:1892-1896.

142. Takahashi N, Yamamoto A, Shingo T, et al. Assessment of left ventricular dyssynchrony during development of heart failure by a novel program using ECG-gated myocardial perfusion SPECT. Circulation Journal. 2008;72:370-377. 
143. Nakamura K, Takami M, Shimabukuro M, et al. Effective prediction of response to cardiac resynchronization therapy using a novel program of gated myocardial perfusion single photon emission computed tomography. Europace. 2011;13:1731-1737.

144. Jiménez-Ángeles L, Valdés-Cristerna R, Vallejo E, Bialostozky D, MedinaBañuelos V. Factorial phase analysis of ventricular contraction using equilibrium radionuclide angiography images. Biomedical Signal Processing and Control. 2009;4:149-161.

145. Jain AK. Data clustering: A review. ACM computing surveys. 1999;31:316-323.

146. Du K-. Clustering: A neural network approach. Neural Networks. 2010;23:89-107.

147. Kimura Y. Improved signal-to-noise ratio in parametric images by cluster analysis. Neuroimage. 1999;9:554-561.

148. Domenichelli S. Quantitative cardiac dynamic imaging of small animal PET images using cluster analysis. Computers in Cardiology. 2008;35:337-340.

149. Wong K-. Segmentation of dynamic PET images using cluster analysis. IEEE Trans Nucl Sci. 2002;49:200-207.

150. Clark MC. MRI segmentation using fuzzy clustering techniques. IEEE Engineering in Medicine and Biology Magazine. 1994;13:730-742.

151. Goutte C. On clustering fMRI time series. Neuroimage. 1999;9:298-310. 
152. Baumgartner R. Fuzzy clustering of gradient-echo functional MRI in the human visual cortex. part I: Reproducibility. Journal of magnetic resonance imaging. 1997;7:1094-1101. 